\title{
IDENTIFYING THE CRITICAL FACTORS IN SOFTWARE DEVELOPMENT METHODOLOGY FIT
}

by

Julien Lemétayer

A thesis submitted to the Victoria University of Wellington in partial fulfilment of the requirements for the degree of Master of Management Studies

Victoria University of Wellington

2010 


\begin{abstract}
In software development, one size does not fit all. Contingencies shape the alignment between the project and its environment, and between software development and project success. Yet the conditions favouring a particular software development methodology (e.g., waterfall or scrum) are not well understood. The current research aims to answer two questions: (1) What are the important factors in software development methodology (SDM) fit? (2) What is the role of SDM fit in project success?
\end{abstract}

A review of the IT literature revealed two kinds of SDMs. On the one hand, there are traditional, plan-driven methodologies that seek compliance to a pre-established plan and existing processes. On the other hand, there are agile methodologies that seek to embrace the increasing changes and uncertainty involved in software development projects. The literature review established that there is no agreement on the contingencies associated with the use of each methodology, nor agreement on how to measure project success.

Exploratory research was undertaken to identify contingencies in software development. Preliminary interviews of projects workers, using a card sort procedure, helped to identify key constructs and to generate and refine a set of measurement items. Then an international survey of software development project workers was conducted.

Data analysis revealed two factors that are important in SDM fit: one is organizational culture; and the other is empowerment of the project team. The first factor encapsulates variables related to the project environment such as the level of entrepreneurship and methodology supported by top management. The second factor is related to the characteristics of the project and includes variables such as procedural empowerment and project uncertainty.

No support was found for factors such as project size, criticality and the need for personnel supervision that are generally considered important contingencies. The current study also demonstrates that SDM fit is one of the predictors of project success, and affirms prior claims that one methodology does not fit all projects.

The current research contributes to the SDM fit literature a contingency model that includes the impact of factors associated with the project and the project environment, on SDM fit and project success. The contingencies identified and evaluated by this research may assist practitioners to select the most appropriate methodology, and to achieve higher project success rates. 


\section{ACKNOWLEDGEMENTS}

I would like to acknowledge all the people who provided me with support, confidence and assistance in undertaking and completing this master's thesis.

Firstly, I would like to thank my supervisor, Dr Jim Sheffield, without whom this work would not have been possible. He provided me with valuable insights and helped me structure this research. Our 'Agile' way of working allowed us to achieve good quality research. His generosity of time and effort was invaluable.

Secondly, I would like to thank academics, PhD students and administrative staff from the Victoria Management School and School of Information Management who supported me and provided me with useful guidance.

Thirdly, I would like to thank all the participants in my study (more than 120 people) for taking the time to participate in this research study. This includes Wellingtonians who gave their valuable time to participate in my interviews and world-wide project workers who took my survey.

Next, I would like to thank my university in France (ESIEA) for allowing me to pursue the Master of Management Studies at Victoria University of Wellington. Susan Loubet, the International Relations Manager, deserves my special thanks for her constant support throughout my time in New Zealand. Jean-Paul Vedel should also be thanked for his time.

Lastly, I thank my colleagues, friends and family who have always been on my side throughout this great journey. 


\section{TABLE OF CONTENTS}

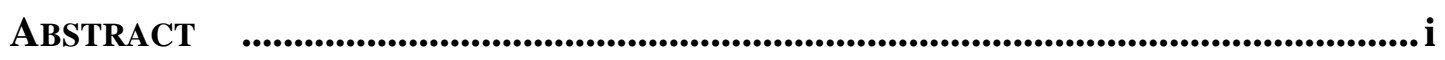

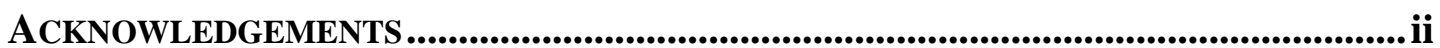

TABLE OF CONTENTS .................................................................................................iii

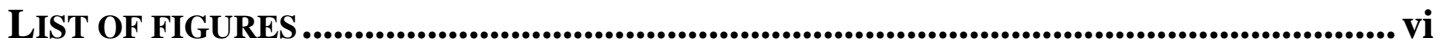

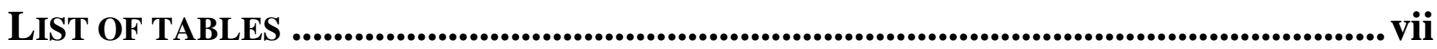

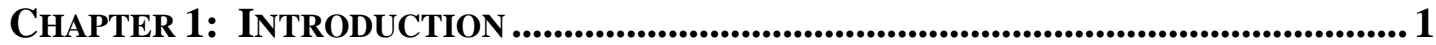

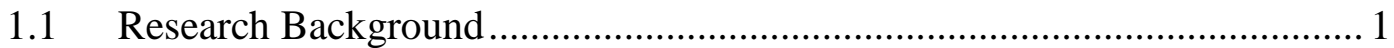

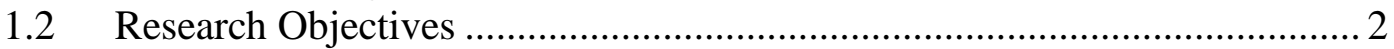

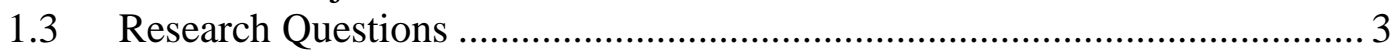

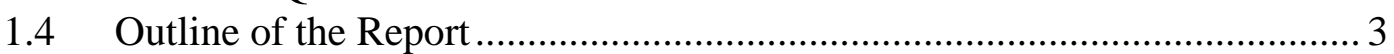

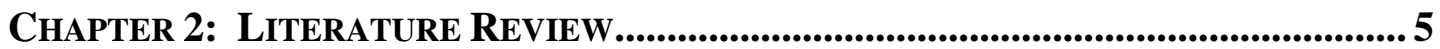

2.1 Introduction - Project Management and Software Development ................ 5

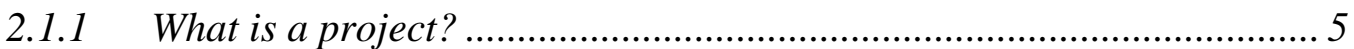

2.1.2 What is software project management? .............................................. 6

2.1.3 What is a project management methodology? ....................................... 7

2.1.4 What is a software development methodology (SDM)? ........................ 8

2.1.5 Why do we need methodologies? ...................................................... 9

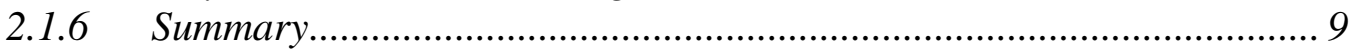

2.2 Traditional Software Development Methodologies .................................. 10

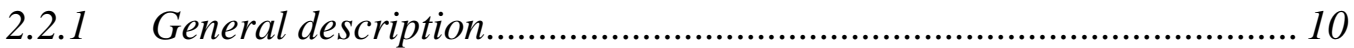

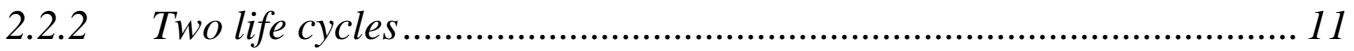

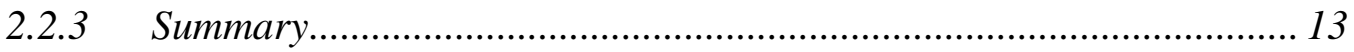

2.3 Agile Software Development Methodologies ............................................ 13

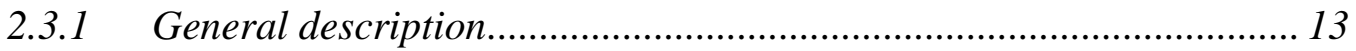

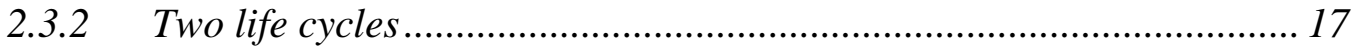

2.3.3 An example of agile software development methodology: Scrum....... 18

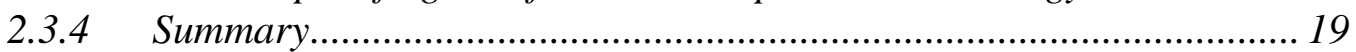

2.4 Comparison of Traditional and Agile Approaches..................................... 20

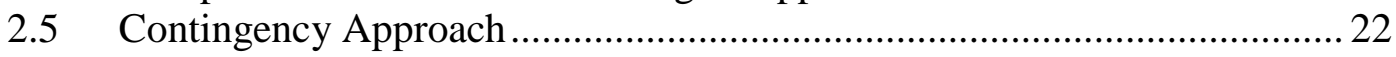

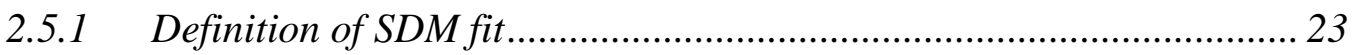

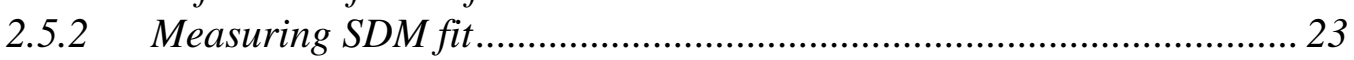

2.5.3 Software development methodology fit .......................................... 26

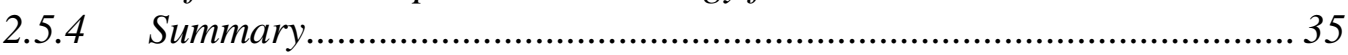

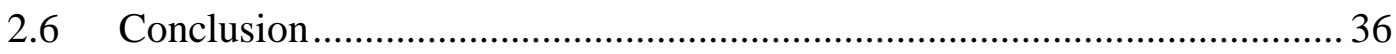




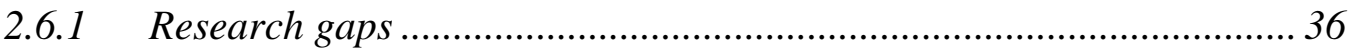

2.6.2 Description of the research model ..................................................... 37

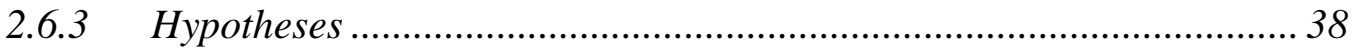

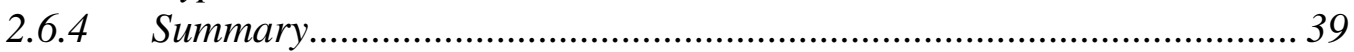

ChaPTER 3: Methodology .......................................................................................... 40

3.1 Operationalization of the Research Model ............................................... 40

3.1.1 Description of the five clusters......................................................... 40

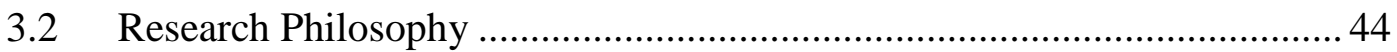

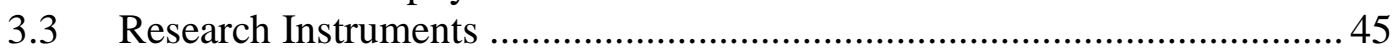

3.3.1 Semi-structured interviews................................................................ 46

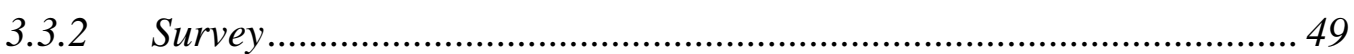

3.4 Credibility of the Research and Generalizability ...................................... 56

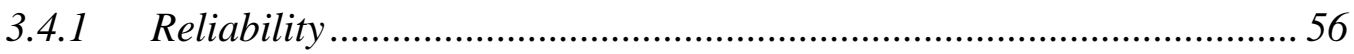

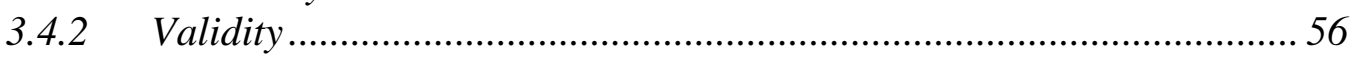

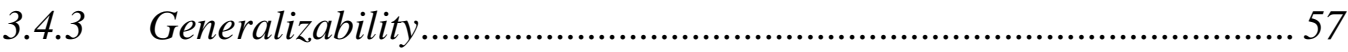

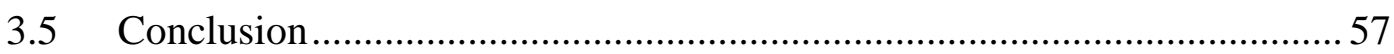

ChAPTER 4: DATA ANALYSIS AND RESUlTS ...............................................................59

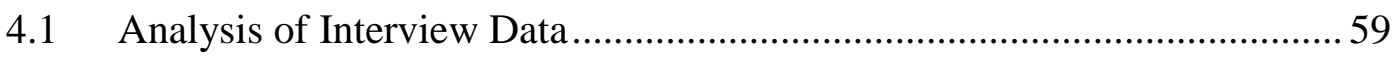

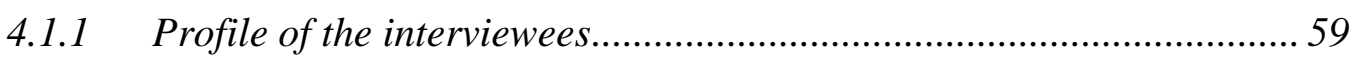

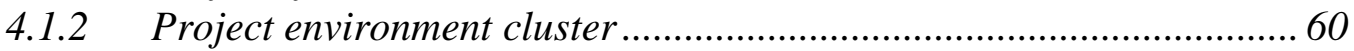

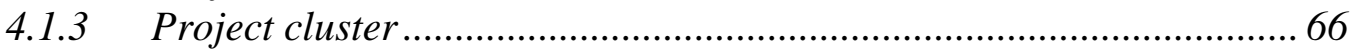

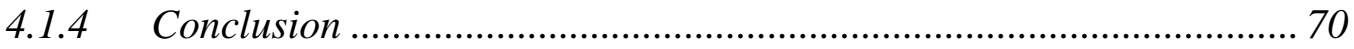

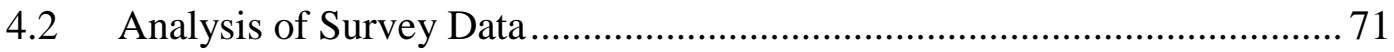

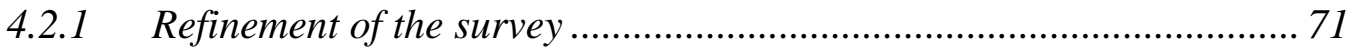

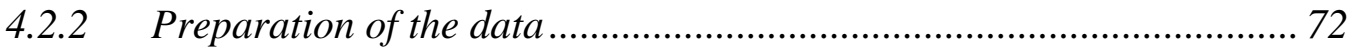

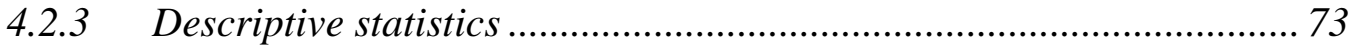

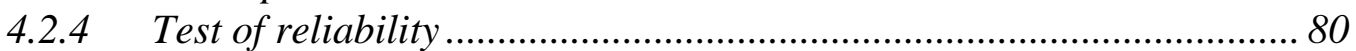

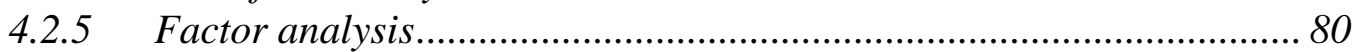

4.2.6 Correlation analyses on the whole database ....................................... 83

4.2.7 Analysis of the SDM fit model ............................................................. 86

4.2.8 Analysis of the project success model ............................................... 90

4.2.9 Limitations of data analysis and validity ......................................... 93

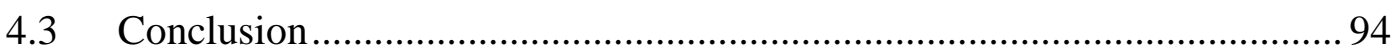

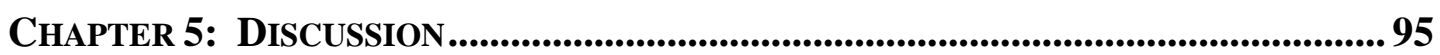

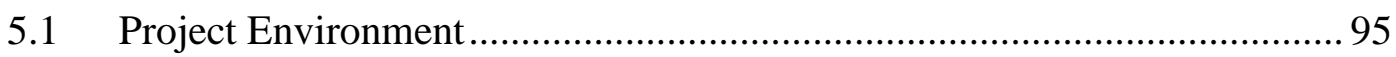

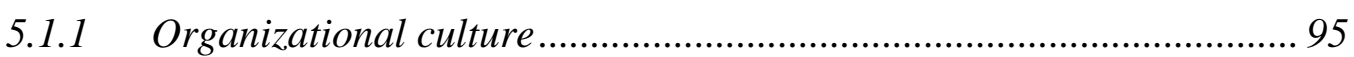

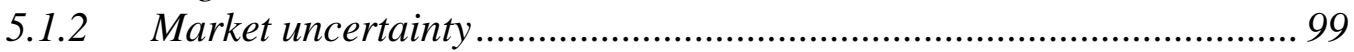

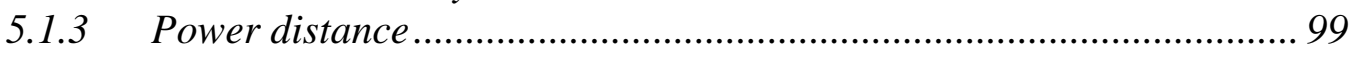

5.1.4 Other project environment variables ............................................... 100

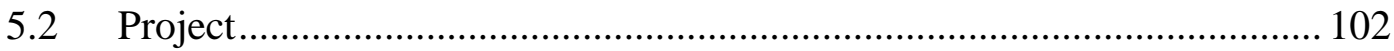


5.2.1 Empowerment of the project team..................................................... 102

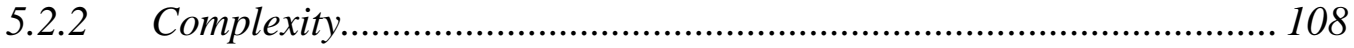

5.2.3 Experience level of the team ......................................................... 110

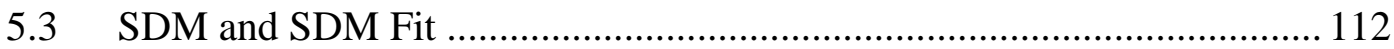

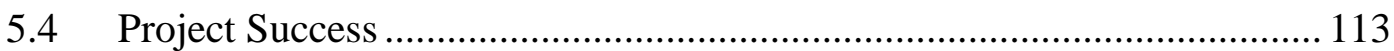

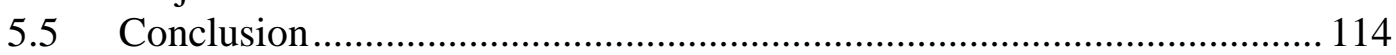

Chapter 6: CONCLUSION........................................................................................ 115

6.1 Responses to the Research Questions ................................................... 115

6.1.1 Critical factors in SDM fit ............................................................... 115

6.1.2 Role of SDM fit in project success ................................................ 117

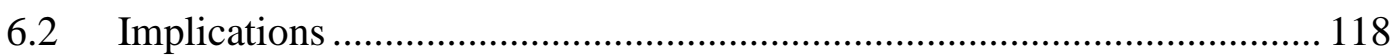

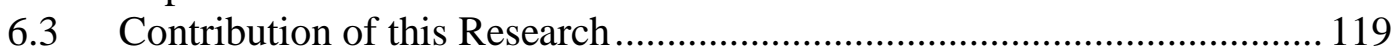

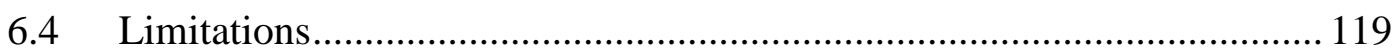

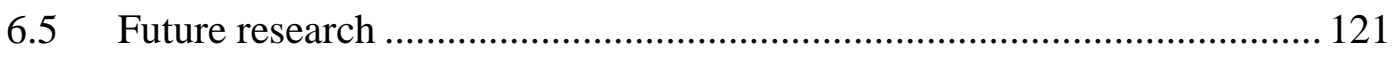

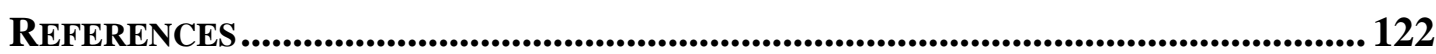

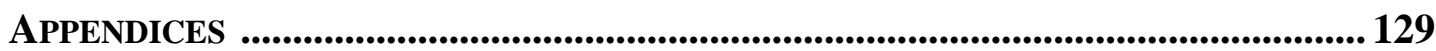

Appendix A: Information sheet given to interview participants ........................ 130

Appendix B: Research agreement signed by interviewees ................................ 131

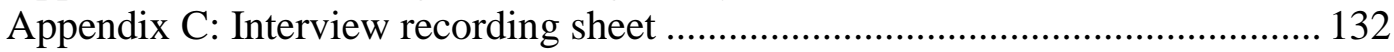

Appendix D: Information sheet sent to survey sample ................................... 133

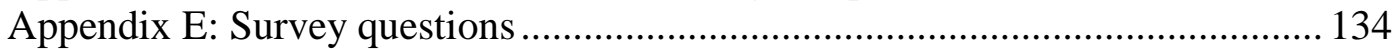

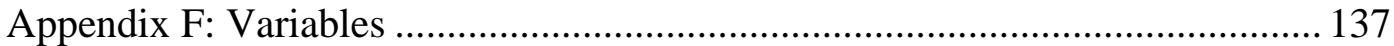




\section{LIST OF FIGURES}

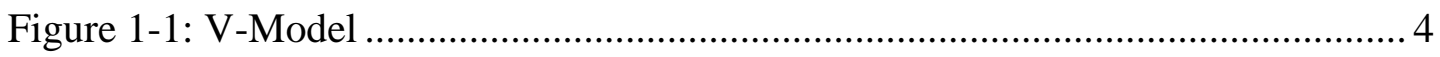

Figure 2-1: The project management hierarchy and associated methodologies .......... 6

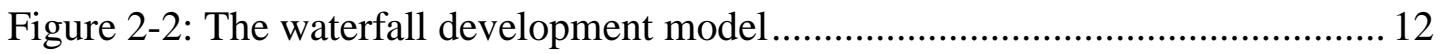

Figure 2-3: The Agile Manifesto ....................................................................... 15

Figure 2-4: Declaration of Interdependence ...................................................... 16

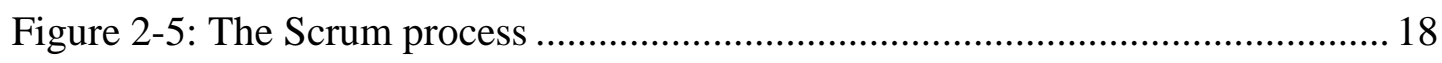

Figure 2-6: Balancing optimizing and adapting dimensions ............................... 21

Figure 2-7: Factors that discriminate between agile and plan-driven methodologies .. 27

Figure 2-8: Problem size, number of people needed, and methodology choice ........ 30

Figure 2-9: The contemporary software development landscape ............................ 33

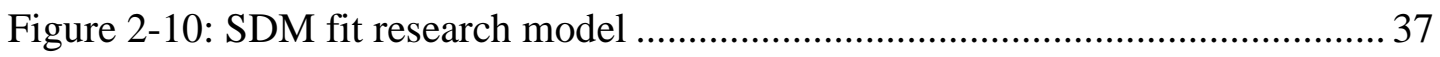

Figure 2-11: Project success research model ….................................................. 38

Figure 4-1: Regression analysis summary (SDM fit research model) ...................... 90

Figure 4-2: Regression analysis summary (project success research model) ............93

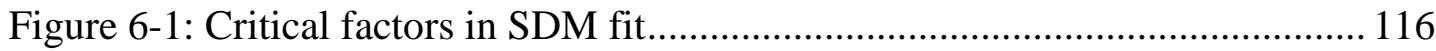

Figure 6-2: Critical factors in project success ................................................... 117 


\section{LIST OF TABLES}

Table 2-1: Traditional and agile perspectives on software development

Table 2-2: How previous studies on methodology selection or implementation measured success 25

Table 2-3: Contingency factors in SDM fit. 32

Table 2-4: Summary of contingency factors identified in the literature 35

Table 2-5: Main research gaps 37

Table 3-1: Project environment variables 41

Table 3-2: Project variables 43

Table 3-3: SDM variables .43

Table 3-4: Project success variables .44

Table 4-1: Interviewees' profile .59

Table 4-2: Ranked project environment variables 60

Table 4-3: Ranked external environment variables 61

Table 4-4: Ranked organizational variables. 62

Table 4-5: Ranked project variables 66

Table 4-6: Ranked criticality variables 66

Table 4-8: Ranked personnel variables 67

Table 4-9: Ranked dynamism variables 67

Table 4-10: Ranked culture variables 68

Table 4-11: Ranked people management variables 69

Table 4-12: Dependent variables to be measured in the survey. 71

Table 4-13: Codes used for the project cost and project size variables 72

Table 4-14: Characteristics of the survey sample .75

Table 4-15: Participants by country .77

Table 4-16: Summarized descriptive statistics for the independent variables 78 
Table 4-17: Summarized descriptive statistics for the dependent variables 79

Table 4-18: Reliability analysis 80

Table 4-19: Factor analysis of the project environment variables 81

Table 4-20: Factor analysis of the project variables 82

Table 4-21: Factor analysis of the SDM variables 83

Table 4-22: Factor analysis of the project success variables .83

Table 4-23: Correlation analysis between the independent variables ..... .84

Table 4-24: Correlation analysis between the independent factors and SDM 85

Table 4-25: Correlations of the independent factors with SDM (SDM fit research model) .86

Table 4-26: Regression coefficients (SDM fit research model). .89

Table 4-27: Regression model summary (SDM fit research model) .89

Table 4-28: Correlations with project success (project success research model) ...... 91

Table 4-28: Regression coefficients (project success research model). 92

Table 4-30: Regression model summary (project success research model) 93

Table 5-1: Project environment factors and their associated variables 95

Table 5-2: Project factors and their associated variables 102 


\section{CHAPTER 1: INTRODUCTION}

This first chapter presents the background and context of the research area as well as the practical problem that this research study investigates. It also presents the motivations behind the choice of this topic. Lastly, it details the structure of this report from the problem investigated, to the findings and contribution to the research area.

\subsection{Research Background}

Leffingwell (2007) describes the software industry as one of the most important of our time. Indeed, software is used by most organizations worldwide. The software industry has become very dynamic and is constantly evolving, in particular with the predominance of the internet and new technologies. High change, high speed, uncertainty and complexity are becoming the characteristics of many projects (Atkinson, Crawford, \& Ward, 2006; Winter, Smith, Morris, \& Cicmil, 2006). Global competition is also increasing (Pixton, Nickolaisen, Little, \& McDonald, 2009). Thus the ability to quickly create and deliver software that meets customers' real needs has become an undeniable competitive advantage (Leffingwell, 2007). Selecting the right software development methodology (SDM) has become essential to meet requirements for cost, quality, and project schedules (Charvat, 2003). Conversely the choice of an inappropriate methodology increases project risk and slows the project (Elkington \& Smallman, 2002; McConnell, 1996). This is reflected by the 'one size does not fit all' principle, which is now widely accepted (Charvat, 2003; Wysocki, 2009). In other words, different projects require different development methodologies (Charvat, 2003). However, because numerous SDMs are available on the market, it can be difficult to select the most appropriate methodology. Moreover, people tend to be emotional about methodology and biased towards one approach in particular (Boehm \& Turner, 2004).

Project management has existed in its modern form since the 1950s. The first development methodologies were developed in the engineering and construction world. These methodologies, known as traditional, are characterized by a plan-driven 
development approach. Project management is traditionally characterized by the iron triangle, showing the interrelations of scope, time and money.

More recently, alternative agile methodologies have emerged to respond to increasing changes and uncertainty in the environment. These methodologies, which are specific to software development, are becoming increasingly popular. This has led to the current debate in the literature about when to choose an agile or traditional approach. At present, there is no accepted way to unambiguously characterize project characteristics and then match them to an appropriate management and development style (Sauser, Reilly, \& Shenhar, 2009).

Lastly, communities of practice like the Project Management Institute (PMI) or the Agile Alliance promote good practices. Yet, IT projects continue to fail in terms of budget overrun, late delivery and failure to achieve objectives (The Standish Group, 2001). The old SDMs are often considered to be too rigid to fit the new environment. However, some project managers still try to force fit them to the wrong projects and fail to consider alternatives (Howell, Windahl, \& Seidel, 2010). Despite exhortation to move away from old practices, the new methodologies are not silver bullets that will guarantee success every time (Highsmith, 2010).

\subsection{Research Objectives}

Many projects fail to deliver on scope, time or budget, or they deliver a product that does not match the client's needs (Sauser et al., 2009). There can be many reasons why a project fails. One of them is the incorrect choice of the SDM (Charvat, 2003; Sauser et al., 2009). Tiwana and Keil (2004), who studied 720 software projects, found that the use of an inappropriate methodology is the most critical risk driver. Therefore, objective guidance in matching the project type and the software development approach is expected to increase the chances of project success, and to reduce the risk of project failure. Howell et al. (2010) explain that the lack of a decision support tool discourages project managers from considering alternative methodologies. In addition, the actual impact of the best fit methodology on project success is not well understood. 
The aim of this research is to engage with members of diverse software development communities so as to identify the contingency factors in the selection of a SDM that fits the characteristics of a particular project. A quantitative measure of SDM fit will be developed and the importance of the contingency factors will be objectively determined. Finally, this research aims at evaluating the effect of SDM fit (i.e. the choice of the most appropriate SDM) on project success.

\subsection{Research Questions}

The research aims stated above lead to the following broad research questions:

- What are the critical factors in SDM fit?

- What is the role of SDM fit in project success?

\subsection{Outline of the Report}

This thesis is structured in six chapters following the V-Model (Sheffield, 2005; Figure 1-1). This figure shows how the different chapters of this report fit together. Research intentions appear on the left and research outcomes appear on the right. The purpose of the model is to ensure alignment between intentions and outcomes at three levels of abstraction, which are simply labelled 'Why', 'What' and 'How'.

This first chapter describes the initial broad research problem and why it is a topic of interest. It also presents the research aims, research questions, and the outline of the report.

The second chapter - literature review - looks at the work that has been undertaken in the field. It describes the two main approaches in software development. It also reviews the principles and contingency factors in SDM fit and success. The aim of this chapter is to highlight research gaps and develop a research model.

Chapter three - methodology - informs the reader about the research philosophy and methodologies (interview and survey) used to gather empirical data and test the 
research model. It also carefully describes the key constructs that are measured and develops hypotheses. This is the basis for the data analysis presented in chapter four.

The next chapter - data analysis and results - reports the analysis of data gathered from the interviews and from the survey. The results of statistical analyses of the data as well as illustrative graphs and tables are presented and are used as a basis for the following chapter.

Chapter five - discussion - interprets and discusses the data analysis and then relates the findings to the gaps in the existing literature (chapter two). This chapter reviews the research model and explains the implications of the findings.

Last of all, chapter six concludes this report with a summary of the key findings and qualified responses to the initial broad research questions. It also acknowledges the limitation of this research and gives recommendations for future research.

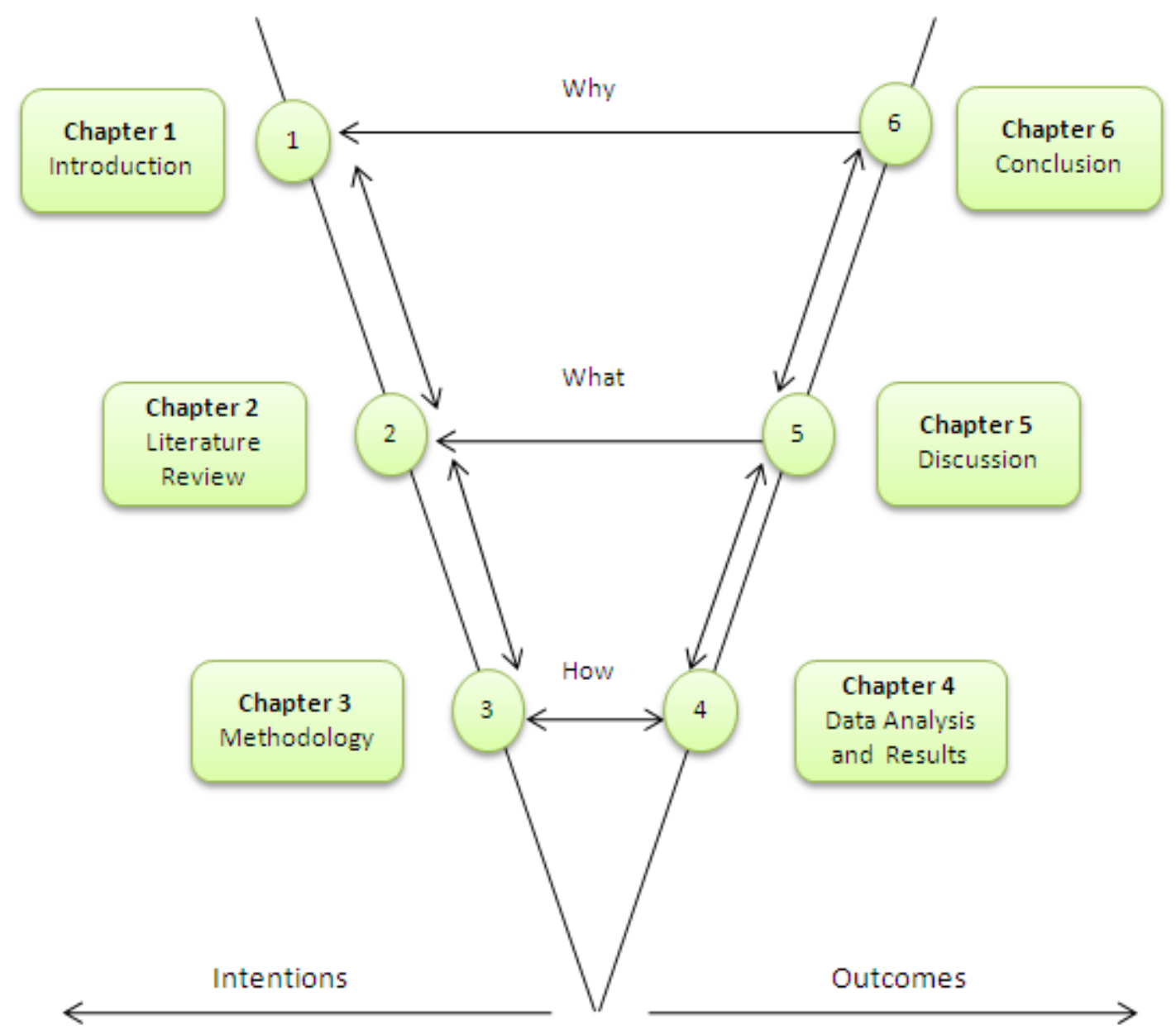

Figure 1-1: V-Model (Adapted from Sheffield, 2005) 


\section{CHAPTER 2: LITERATURE REVIEW}

This chapter presents the literature on the topic of SDMs and methodology selection and fit. After an introduction on IT project management, a description of the two main development approaches is given. These are compared and contrasted. A large part of this chapter looks at the contingency factors presented in the literature that should guide the selection of a SDM. The concept of project success is also investigated. This chapter ends with a presentation of the research gaps that have been highlighted from literatures searches and a presentation of the research model.

\subsection{Introduction - Project Management and Software Development}

\subsubsection{What is a project?}

A project is usually defined as "a temporary endeavour undertaken to create a unique product, service, or result" (PMI, 2008, p. 5). This definition implies that a project has a purpose and it exists to achieve some specific outcomes (Dalcher \& Brodie, 2007). It is a unique undertaking as opposed to routine work. Finally, it is a temporary activity that has a beginning (start date) and an end (deadline), and it needs resources (for example, people and money) to be completed. Projects are now a central activity in many organizations, which invest a lot of resources in them to drive innovation and change (Sauser et al., 2009; Shenhar \& Dvir, 2007).

A software development project can be defined as "a complex undertaking by two or more persons within the boundaries of time, budget, and staff resources that produces new or enhanced computer code that adds significant business value to a new or existing business process" (Wysocki, 2006, p. 5)

Projects do not exist in a vacuum but take place in an organizational context, which influences them (APM, 2006). In particular, projects may be part of a programme (Pellegrinelli, 2010), which can be defined as "a group of related projects managed in a coordinated way to obtain benefits and control not available from managing them individually" (PMI, 2008, p. 16). Then at a broader level, all the individual projects 
and programs conducted in an organization or organizational unit are brought together in a portfolio (APM, 2006).

Each level uses its own management methodology. This study particularly focuses on the software development level and SDMs (Figure 2-1). The project management methodologies that have a close relationship with SDMs are briefly discussed in this report.

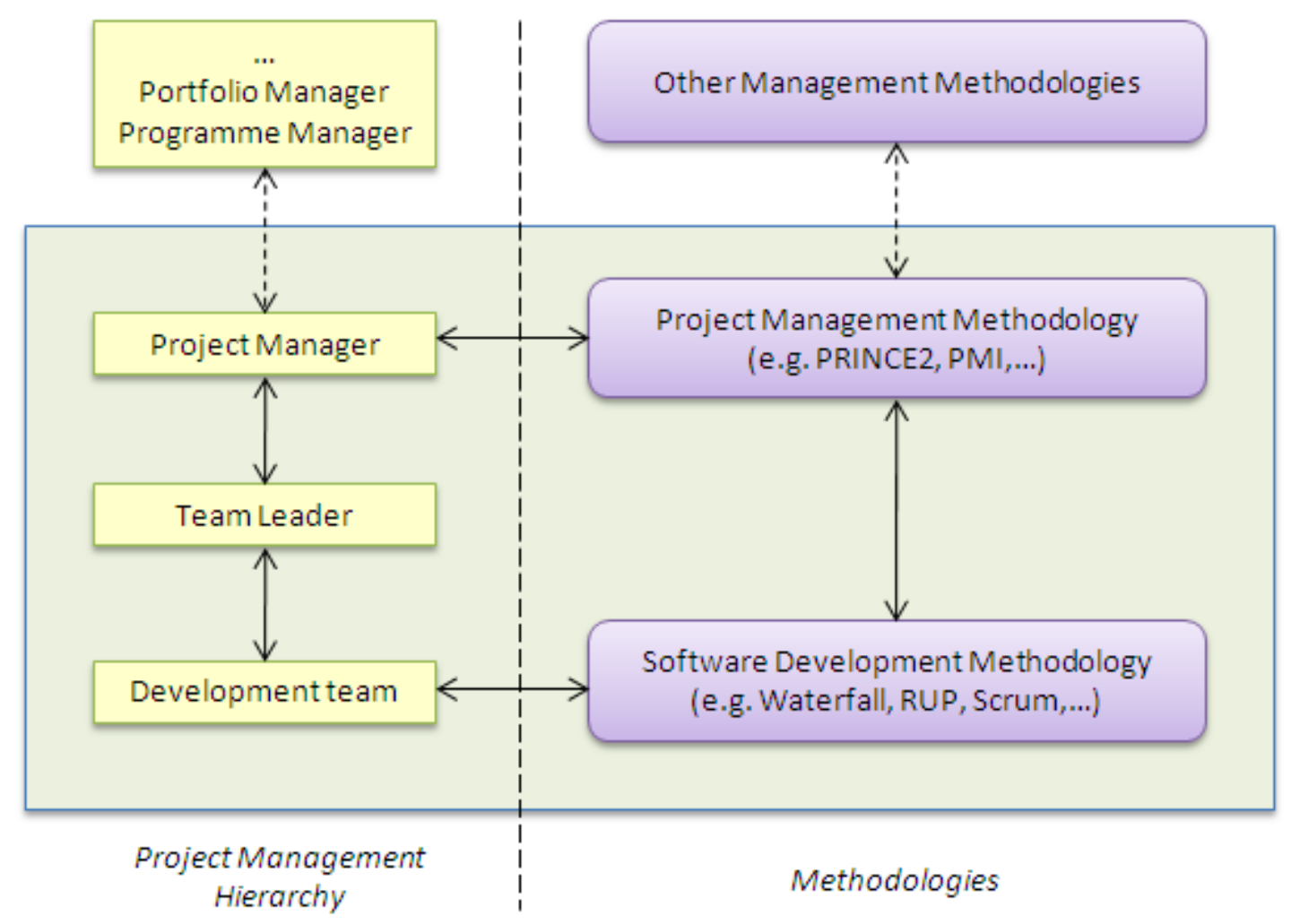

Figure 2-1: The project management hierarchy and associated methodologies (adapted from APM, 2006, p.7; Charvat, 2003, p. 7; Dalcher \& Brodie, 2007, p. 10; OGC, 2009, p. 219)

\subsubsection{What is software project management?}

Project management is defined by the Project Management Body Of Knowledge (PMBOK) guide (PMI, 2008, p. 6) as "the application of knowledge, skills, tools, and techniques to project activities to meet the project requirements". Even though good project management cannot by itself guarantee project success, because many other factors also influence the outcome of a project, bad management often results in project failure (Sommerville, 2006 cited in Dalcher \& Brodie, 2007). 
Software project management can present some difficulties. Unlike the tangible progress of constructing a building, software progress is hard to see and is therefore largely intangible. Moreover, in IT the technology changes more rapidly than in other industries and the requirements are not always stable. Lastly, the skills of the IT project manager may quickly become obsolete if they are not updated (Dalcher \& Brodie, 2007).

\subsubsection{What is a project management methodology?}

The Project Management Institute (PMI, 2008, p. 438) defines a methodology as "a system of practices, techniques, procedures, and rules used by those who work in a discipline". Similarly, Charvat (2003, p. 3) defines a methodology as "a set of guidelines or principles that can be tailored and applied to a specific situation". Thus it can only be a list of things to do or more specifically an organized, documented set of rule and practices including processes, templates, forms or checklists used over the project life cycle. As Cockburn (2007) points out, there is a distinction between a methodology and method. He cites the definition of a methodology found in the Merriam-Webster dictionary: "A series of related methods or techniques" (Cockburn, 2007, p. 149). This definition is consistent with Avison and Fitzgerald (1995) who argue that the term methodology is a wider concept than method. Methods are related to techniques and procedures, while methodologies address broader issues including the coordination of activities. Without coordination, cooperation, and communication, even a group of smart, talented individuals will not produce good results.

Project management methodologies lay the high-level framework of the project (Charvat, 2003). They are often organized in phases from initiating to closing the project. Winter, Smith, Morris, and Cicmil (2006, p. 640) assert that "the most dominant strand of project management thinking is the rational, universal, deterministic model... emphasizing the planning and control dimensions of project management". This strand is represented by communities of practice like PRINCE2 ${ }^{1}$ and PMI (PMBOK), which tend to favour traditional SDMs. However, this view is

\footnotetext{
${ }^{1}$ PRINCE2 is an abbreviation of PRojects IN Controlled Environments
} 
challenged by others who subscribe to alternative approaches such as agile. Thus the project management field is not a homogeneous one characterized by a common set of principles, guidelines and practices. Rather it is composed of many different practices, some ad hoc, and some developed within communities whose members share certain methodological commitments. Nevertheless, the latest versions of the PRINCE2 manuals and PMI's PMBOK acknowledge the rise of agile SDMs and now accommodate these SDMs within their project management style (OGC, 2009; PMI, 2008).

\subsubsection{What is a software development methodology (SDM)?}

Huisman and Iivari (2006) explain that defining a SDM is not an easy task. In this study, Charvat's broad definition (2003) is used. He explains that SDMs provide the detail that informs software design and development. SDMs typically cover the architecture, development and testing of the system, but does not cover project management issues such as financial justification of the project or sales. SDMs are mapped onto related project management methodologies (Figure 2-1) so that information can flow between them. It is therefore important to ensure that the project management and SDMs are compatible (Dalcher \& Brodie, 2007).

There are many SDMs on the market and their number keeps increasing. In the SDM ecosystem, authors (Boehm \& Turner, 2003; Charvat, 2003; Highsmith, 2010; Wysocki, 2009) usually distinguish two approaches: the traditional or heavyweight approach and the agile or lightweight approach, both of which are reviewed in this report.

Example of heavyweight SDMs include SSADM (Eva, 1994); Information Engineering (Martin \& Finkelstein, 1981); Unified Software Development Process (Jacobson, Booch, \& Rumbaugh, 1999); and OPEN (Graham, Henderson-Sellers, \& Younessi, 1997). On the other end of the planning spectrum, agile methodologies include XP (Beck, 2000); Scrum (Schwaber \& Beedle, 2002); Crystal (Cockburn, 2002); and DSDM (Stapleton, 1997) among others. 


\subsubsection{Why do we need methodologies?}

A methodology is important to respond to the needs of an often dynamic and rapidly changing market. Regardless of the size of the project, a methodology provides a consistent framework through the life cycle of the project and optimizes the performance of the team (Charvat, 2003). The usage of a SDM has been shown to increase both the productivity and the quality of software development (Dybå, Moe, \& Arisholm, 2005). It also defines a common vocabulary, common formats and a strategy for managing the project (Cockburn, 2007). Moreover, when new people are introduced or substituted in the team, the methodology informs them about how work is done on the project and the methodology usually delineates responsibilities (Cockburn, 2007). Without a methodology, the risk of failure is significantly increased. Although methodologies are not a panacea to all IT project problems, the evidence is that they increase productivity and quality while reducing time and effort (Riemenschneider, Hardgrave, \& Davis, 2002).

As projects are now found in most organizations, preventing their failure has become essential (Van Donk \& Molloy, 2008). Choosing the right approach to reduce the risk of failure becomes important if not vital (Charvat, 2003). With an inappropriate methodology, the outcome of the project may be very uncertain. Nonetheless, in many organizations the methodology is imposed at a high level (Figure 2-1) and the project manager can only try to tailor it. Alternatively, project managers naturally select the methodology they have the most experience in and/or the one they feel more comfortable with (Charvat, 2003).

\subsubsection{Summary}

There is a growing diversity of SDMs on the market and their number keeps increasing. Understanding them and their link with project management methodologies is important to the selection of the most appropriate SDM.

In the next sections, the two categories of SDMs are reviewed. Their advantages and drawbacks are examined in order to get a better understanding of when they are most appropriate. These two methodologies are then compared along several dimensions. 


\subsection{Traditional Software Development Methodologies}

\subsubsection{General description}

Traditional methodologies were defined and developed in the 1950s in the world of engineering and construction where complete and accurate requirements from the client were provided. These methodologies, which employ a requirement-designbuild paradigm, are considered to be the traditional way to develop software (Tortamış, 2004). They are associated with well-defined processes (for example, risk management, quality assurance) that are continuously improved. In this kind of project, the requirements are clearly specified at the beginning and little change is expected. Thus the environment is predictable and planning tools can be used to optimize the management of the project, which is why these methods are also known as plan-driven (Boehm \& Turner, 2004). Plan-driven methodologies are usually change-resistant and focus on compliance to plan as a measure of success (Wysocki, 2009). Consequently, they are somewhat prescriptive, and heavy on process and documentation. This heavy reliance on documentation is essential in major projects to jog the memory of the team members, coordinate people (particularly when they are in different locations), and to gain control on critical projects (Charvat, 2003). However, too much documentation may be costly and reduce the productivity of the team workers (Cockburn, 2007).

In brief, the traditional approach works well in the following conditions (Leffingwell, 2007):

- The requirements are well defined

- Change to these requirements are small or nonexistent

- We can accurately predict the necessary tasks and their durations.

Hence, the goal and the solution of the project should be clearly defined from the outset of the project to adopt this kind of approach. The technology used should also be well known or familiar to the project team, so that there are no surprises (Wysocki, 2009). 
The main risks of adopting plan-driven approaches relate to (Boehm \& Turner, 2003):

- Rapid change

- Need for rapid results

- Emergent requirements

- Lack of people skilled in plan-driven methods.

The traditional approaches rely on a linear or incremental life cycle as described below.

\subsubsection{Two life cycles}

\subsubsection{Linear model}

In the linear - or sequential - life cycle, the project is completed in one unique cycle. Each stage of the project (from analysis to support) is executed once. The project moves from one stage to the following when the predefined milestones or objectives are achieved. At the end of each stage, the deliverable is not the software itself but the documentation (for example, business requirements or design). The waterfall model is a well known example of a linear model (Figure 2-2).

The waterfall model of development has been in use by the software industry for more than 30 years (Leffingwell, 2007). This represented a significant improvement over ad hoc or "code-and-fix" approaches, which were particularly used at the beginning of the software industry. This model provides a pre-specified list of process steps stretching from the requirement analysis to the delivery of the product to the client. In a pure waterfall implementation, there is a single progression through pre-defined process steps and the activities associated with each of them. Moreover, subsequent phases are not started until the current phase is finished. This approach makes the assumption that all the requirements can be well understood and documented in advance and that change will be negligible during the project. If errors are found early in the development life cycle or if there is change at the beginning of the project, it will be relatively inexpensive to fix. On the contrary, if 
change occurs in a later phase, the cost will be very high. Thus to avoid rework, these methods are compliance-driven. A lot of effort is put in the initial plan, and then the compliance to the plan drives the whole project (Perrin, 2008).

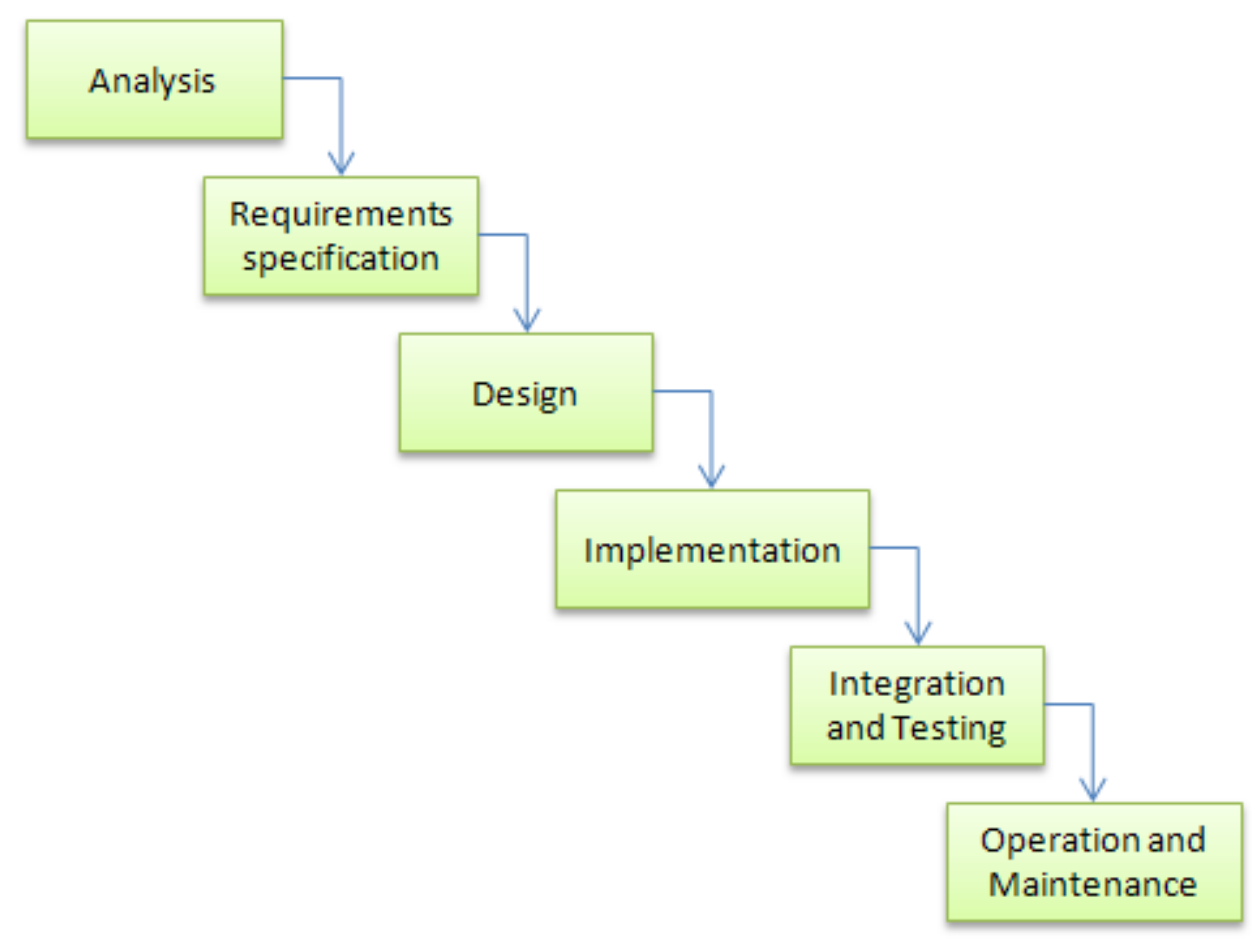

Figure 2-2: The waterfall development model (Adapted from Leffingwell, 2007)

This model is often criticized for its rigidity: it is resistant to change because of high compliance to the initial plan. Any change would lead to change in the budget and timeline. Being responsive to change is important, though, to remain competitive. In addition, change is more and more frequent (Highsmith \& Cockburn, 2001). While thousands of projects using this approach have been successful, many have also been delivered late (Leffingwell, 2007). The integration phase in particular often poses numerous problems. Alternatively, software has often been delivered with functionalities that do not respond to the client's needs. This is due to the fact that the full requirements definition at the beginning of the project is followed by a long period before these are delivered. The client only has a passive role and provides little feedback during the project and there may be a discrepancy between what the client expected and what was delivered. 


\subsubsection{Incremental model}

In the same category of traditional approaches, there are also approaches based on an incremental model. Contrary to the linear model, the development phases (i.e. design, build, and test) are executed more than once. At each increment, the project is expanded according to a pre-specified plan. This allows phased delivery to the client (Charvat, 2003). Even though this approach allows more flexibility, it still follows a pre-determined plan developed at the beginning of the project and compliance to that plan is high.

\subsubsection{Summary}

Traditional approaches are based on a pre-specified plan. In practice, these predictive methodologies have resulted in many unsuccessful projects (70-plus percent according to Wysocki, 2009). Wysocki (2009) also argues that no more than 20 percent of all projects have the characteristics of traditional projects, but project managers continue to apply these traditional ways of developing software on projects for which they are not suited. These methodologies work well until changes occur. These changes do not fit with the compliance and plan-driven approach, which is why project managers tend to be change-resistant. When change is accepted, the plan needs to be updated and previous work become obsolete. Thus time was wasted on tasks that did not add value to the project.

Other names used in the literature for traditional methodologies include plan-driven, rigorous, predictive or heavyweight.

\subsection{Agile Software Development Methodologies}

\subsubsection{General description}

In reality, clients are seldom able to provide complete or stable requirement specifications at the beginning of the project. The rapid changes in the technical and industry-specific environment may prevent the client from accurately predicting their 
needs either long-term or medium-term. If this is the case, the best solution is to choose a methodology that adapts or evolves product characteristics as the client's perceived and/or actual needs change during the development process (Charvat, 2003). These methodologies need to be more flexible than the traditional, rigorous models because organizations need short delivery cycles to cope with uncertainty and rapid change in requirements (Wysocki, 2009). Agile methodologies have been developed to respond to these new constraints and are specifically based on the assumption that the perceived and/or actual requirements will change during the course of the project. Thus agile approaches are designed to accept and embrace change. Such approaches allow the customer to learn about his needs during the process of building the solution. Agile projects require a meaningful client involvement in every part of the project to provide constant feedback in an open and honest way (Wysocki, 2009). This feedback is a key element of agile methodologies, which is why the customer must be committed, knowledgeable, collaborative, representative, and empowered to avoid risk of failure (Boehm, 2002). People are the primary drivers of agile projects and agile teams work best when people are physically close and document preparation and dissemination are largely replaced by face-to-face communication and collaboration (Cockburn \& Highsmith, 2001).

Agile methodologies are value-driven rather than plan-driven and use tacit knowledge between team members in place of heavy documentation. This is the key to agility. In agile methodologies, the major, upfront, one-time planning task is replaced by an iterative and adaptive series of just-in-time tasks that are executed only when needed. According to Wysocki (2009, p. 310), "non-value-added work involves the consumption of resources (usually people and time) on activities that do not add business value to the final product or process". Moreover, agile teams are self organizing and roles and relationships evolve as necessary to meet objectives (Leffingwell, 2007). This provides flexibility and adaptability to the project, enabling the project team to cope more readily with change requests. Moreover, valuing people over process allows for greater creativity.

Agile principles are expressed in a remarkably brief Agile Manifesto (Figure 2-3). In 2001, leaders of different methodologies gathered and collaborated on the development of the Agile Manifesto, which is the common philosophy that underlies 
all agile methodologies (Leffingwell, 2007). In addition to the Manifesto for Agile Software Development, the Declaration of Interdependence is the second primary source for agile value (Highsmith, 2010; Figure 2-4).

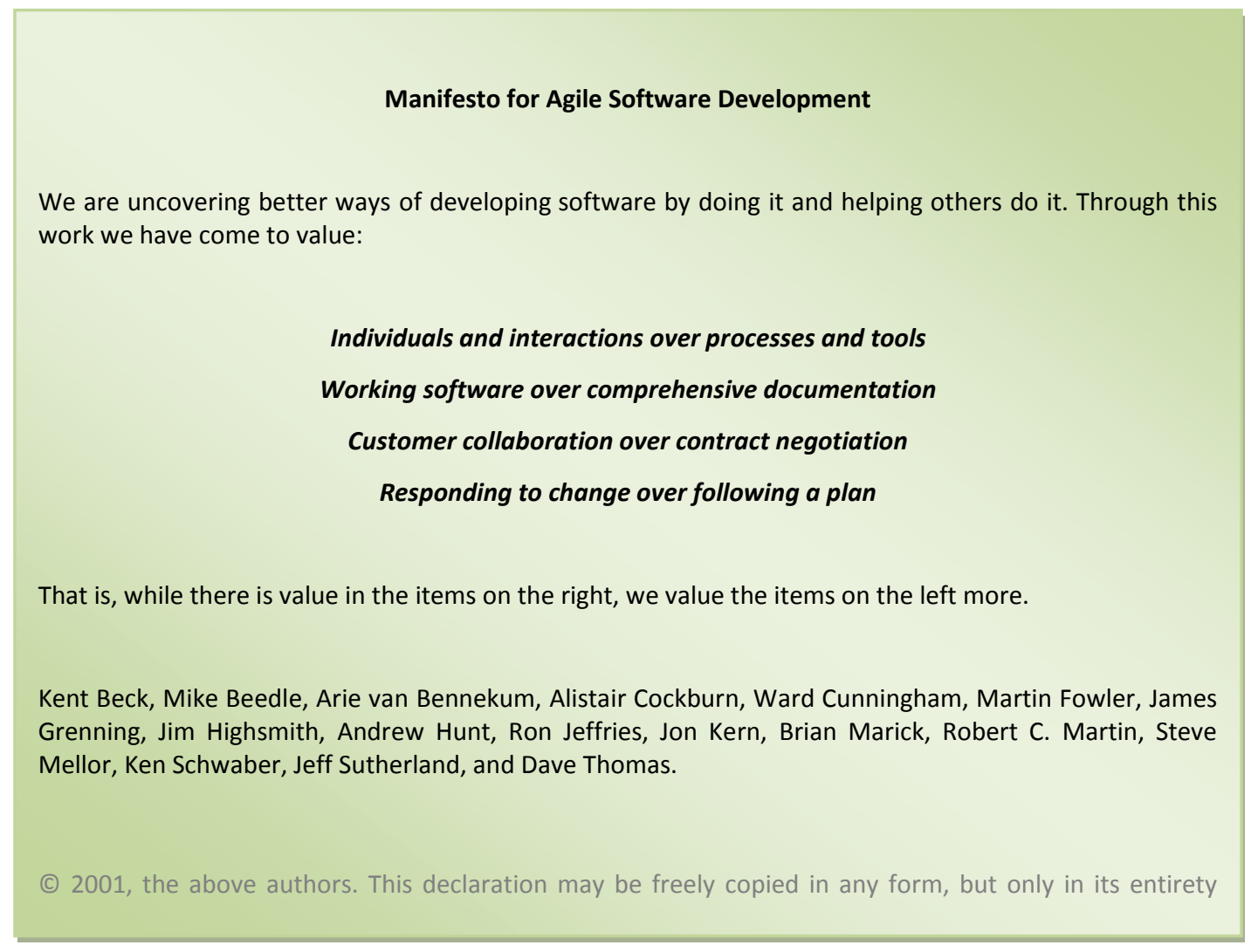

Figure 2-3: The Agile Manifesto (Agile Alliance, 2001)

The Agile Manifesto contrasts traditional methodologies - characterized by the items on the right - and agile methodologies - characterized by the items on the left. The values in the Agile Manifesto are not mutually exclusive; the left and right items can actually reinforce each other. The right items are not considered unimportant but simply less important than the items on the left (Coram \& Bohner, 2005).

The agile manifesto is supported by 12 principles which define the basic philosophy of Agility (Leffingwell, 2007). Highsmith (2010, p. 63) summarizes these principles under the following two statements:

- "We expect change (uncertainty) and respond accordingly rather than follow outdated plans

- We adapt our processes and practices as necessary". 


\section{Declaration of Interdependence}

We are a community of project leaders that are highly successful at delivering results. To achieve these results:

We increase return on investment by making continuous flow of value our focus.

We deliver reliable results by engaging customers in frequent interactions and shared ownership.

We expect uncertainty and manage for it through iterations, anticipation, and adaptation.

We unleash creativity and innovation by recognizing that individuals are the ultimate source of value, and creating an environment where they can make a difference.

We boost performance through group accountability for results and shared responsibility for team effectiveness.

We improve effectiveness and reliability through situationally specific strategies, processes and practices.

C2005 David Anderson, Sanjiv Augustine, Christopher Avery, Alistair Cockburn, Mike Cohn, Doug DeCarlo, Donna Fitzgerald, Jim Highsmith, Ole Jepsen, Lowell Lindstrom, Todd Little, Kent McDonald, Pollyanna Pixton, Preston Smith and Robert Wysocki.

Figure 2-4: Declaration of Interdependence (DOI, 2005)

Agile methodologies have numerous advantages (Charvat, 2003; Leffingwell, 2007; Perrin, 2008) including that they:

- Adapt very well to change and dynamism

- Are people-oriented and value-driven, rather than process-oriented and plandriven

- Mitigate risks by demonstrating values and functionalities up front in the development process

- Provide a faster time to market

- Improve productivity (by reducing the amount of documentation)

- Will fail early/quickly and painlessly, if a project is not doable.

On the other hand, the main risks stemming from an agile approach (Boehm \& Turner, 2003) relate to:

- Limited scalability

- Use of (too) simple design, which may cause expensive rework

- Personnel turnover, which means a loss of knowledge

- Lack of people skilled in agile methods. 


\subsubsection{Two life cycles}

Agile methodologies are based on an iterative or adaptive life cycle.

\subsubsection{Iterative model}

The iterative life cycle, contrary to the incremental one, focuses on redoing the project at each iteration. Therefore, at each iteration there is some learning as a result of feedback and the next iteration might change or adapt what has been done before; in contrast, in an incremental development, increments are planned to fit together and follow each other in a pre-specified order. In brief, an increment does not modify previous work (Charvat, 2003), but an iteration may. This is well illustrated by the agile principle of simplicity. This principle states that future features should not be prepared in the current iteration as they are likely to evolve as a natural outcome of the rapid learning experienced on agile projects (Boehm \& Turner, 2004).

Iterative and adaptive life cycles have the advantage of a continual testing throughout the project, which has a positive impact on quality (Charvat, 2003). Agile methodologies suggest short iterations of less than three months and usually around four weeks. Each iteration covers an entire development life cycle (from the requirement specifications of a specific set of functionalities to the testing and release to the client).

\subsubsection{Adaptive model}

This model is a more extreme version of the iterative model, and is recommended when there is a very high degree of uncertainty and complexity, and very little is known about the project. Learning and discovery are major elements of each cycle of adaptive models, which set them apart from iterative models. Thus each cycle addresses task completion for newly defined functions and the discovery of new features and requirements. Unlike the iterative model, where the scope is known but all the functionalities are not, the adaptive model envisages that the scope of the project will change during development. Therefore, each cycle proceeds on a limited 
understanding of the solution and attempts to converge pragmatically on an acceptable solution. The requirements are obtained and altered through a feedback loop as the system develops (Wysocki, 2009).

\subsubsection{An example of agile software development methodology: Scrum}

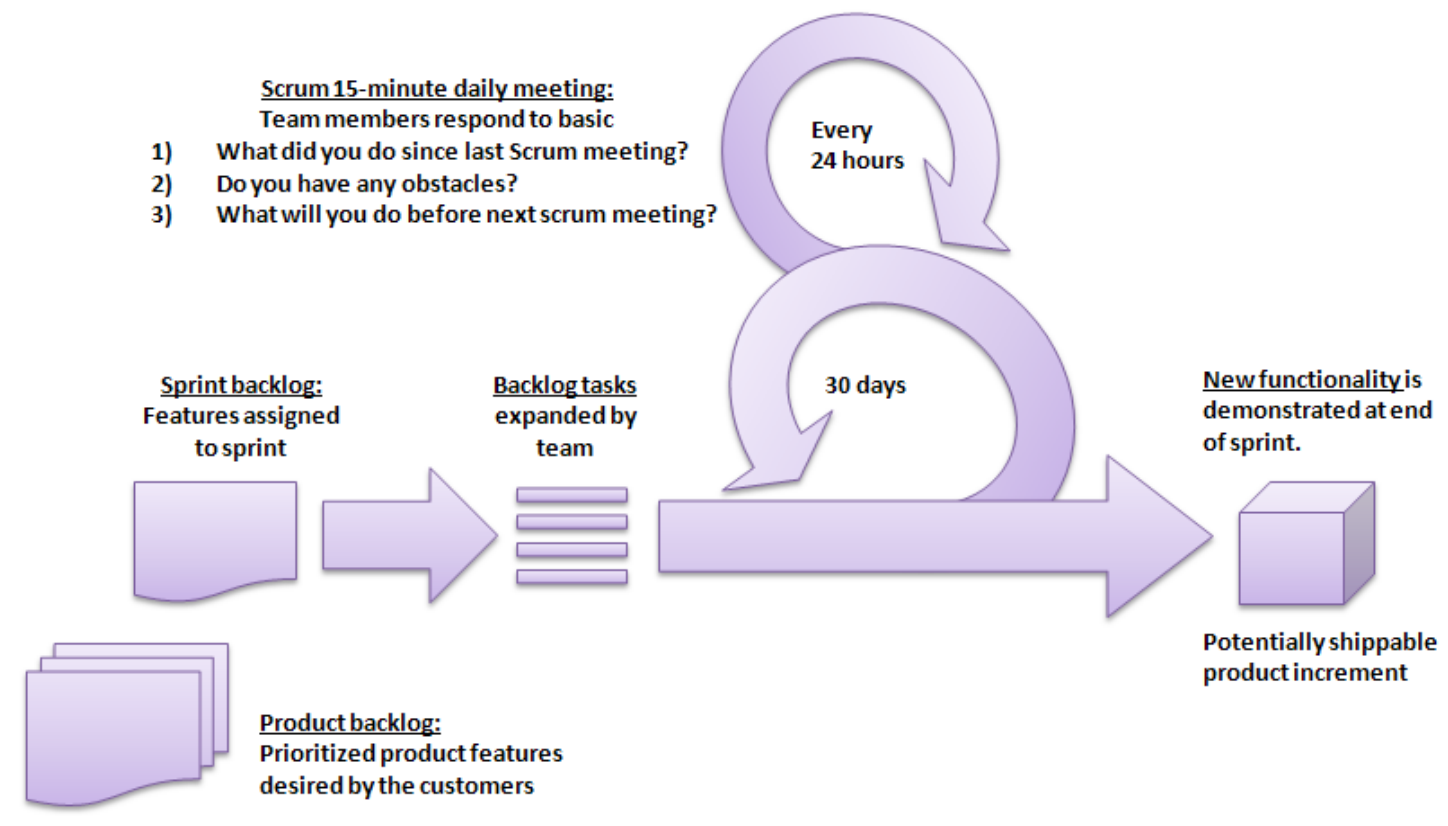

Figure 2-5: The Scrum process (Adapted from Boehm \& Turner, 2005)

This approach was first described by Takeuchi and Nonaka (1986) in "The New New Product Development Game". In Scrum, iterations are called sprints and constitute the core element (Dybå \& Dingsøyr, 2008). Once the scope of the sprint is approved, no additional functionality can be added. In other words, the functions agreed at the beginning of a sprint are considered to be fixed, and are completed, but learning during the sprint is reflected in the product backlog. This backlog, which contains all the features that still need to be implemented, is therefore dynamic, and is reprioritized according to the needs of the customer at the end of each sprint. Features that deliver the most value will have a higher priority and will be developed in the following sprint. All the features are reprioritized as client's needs change.

Each sprint covers all the project stages from planning and architecture to delivery and review. Before the end of a sprint and the delivery, the product is tested. Hence, the software is continuously tested throughout the project, rather than tested only 
once at the end of the project. At the end of the sprint, a working component of the software is delivered to the client who can give feedback to the development team. This feedback will be used to make adjustments to the next sprints if needed (Leffingwell, 2007).

The recommended length of a sprint is 30 days and three sprints are recommended per release. Thus, there is a release every 90 days. Face-to-face communication and implicit knowledge are fostered by daily stand-up meetings. One of the team members, known as the scrum master, is in charge of removing obstacles that could reduce the productivity of the team. Scrum is most effective for teams that have eight or fewer people (Leffingwell, 2007).

\subsubsection{Summary}

Highsmith (2010, p. 17) summarizes the key agile values as follows:

- Valuing "delivering value over meeting constraints"

- Valuing "leading the team over managing tasks"

- Valuing "adapting to change over conforming to plan".

As Highsmith (2010) points out, applying the values is not a matter of mutual exclusivity, but a matter of emphasis. While agile approaches are not a magic bullet, their adoption is growing and they seem to produce better results on some projects (Perrin, 2008). According to a study conducted by Forrester Research in 2009 (West \& Grant, 2010), agile software development processes were in use in $35 \%$ of organizations, and another $16 \%$ of organizations used an iterative development approach, while only $13 \%$ of organization use a Waterfall approach. However, nearly $31 \%$ did not use a formal development methodology.

In practice, the agile approaches are very effective in environments where speed and flexibility are important to success (Perrin, 2008). In these environments, traditional linear methods typically fail. However, over-responding to change has also been cited as the cause of many disasters by Boehm (2002). There is the risk that irrecoverable architectural mistakes are made because of a lack of planning. 


\subsection{Comparison of Traditional and Agile Approaches}

The differences between traditional and agile approaches are summarized in Table 2-1. This lists 14 issues, and provides an indicative source for additional information, albeit often from practitioners who fail to provide empirical support.

\begin{tabular}{|c|c|c|}
\hline Issue & Traditional Approach & Agile Approach \\
\hline $\begin{array}{l}\text { Development life cycle } \\
\text { (Charvat, 2003) }\end{array}$ & Linear or incremental & Iterative or Adaptive \\
\hline $\begin{array}{l}\text { Style of development } \\
\text { (Leffingwell, 2007) }\end{array}$ & Anticipatory & Adaptive \\
\hline $\begin{array}{l}\text { Requirements } \\
\text { (Boehm and Turner, } \\
\text { 2004) }\end{array}$ & Clearly defined and documented & $\begin{array}{l}\text { Emergent - Discovered during } \\
\text { the project }\end{array}$ \\
\hline $\begin{array}{l}\text { Architecture } \\
\text { (Wysocki, 2009) }\end{array}$ & $\begin{array}{l}\text { Heavyweight architecture for } \\
\text { current and future requirements }\end{array}$ & $\begin{array}{l}\text { YAGNI precept (“You aren't going } \\
\text { to need it") }\end{array}$ \\
\hline $\begin{array}{l}\text { Management } \\
\text { (Winter et al, 2006) }\end{array}$ & Process-centric & People-centric \\
\hline \multirow{2}{*}{$\begin{array}{l}\text { Documentation } \\
\text { (Boehm and Turner, } \\
\text { 2005) }\end{array}$} & Heavy / detailed & $\begin{array}{l}\text { Light (replaced by face to face } \\
\text { communication) }\end{array}$ \\
\hline & Explicit knowledge & Tacit knowledge \\
\hline $\begin{array}{l}\text { Goal } \\
\text { (Dybå \& Dingsøyr, 2009) }\end{array}$ & Predictability and optimization & Exploration or adaptation \\
\hline $\begin{array}{l}\text { Change } \\
\text { (Boehm and Turner, } \\
\text { 2003) }\end{array}$ & Tend to be change averse & Embrace change \\
\hline $\begin{array}{l}\text { Team members } \\
\text { (Koch, 2005) }\end{array}$ & Distributed teams of specialists & $\begin{array}{l}\text { Co-location of generalist senior } \\
\text { technical staff }\end{array}$ \\
\hline $\begin{array}{l}\text { Team organization } \\
\text { (Misra et al, 2009) }\end{array}$ & Pre-structured teams & Self-organizing teams \\
\hline \multirow{2}{*}{$\begin{array}{l}\text { Client Involvement } \\
\text { (Highsmith \& Cockburn, } \\
\text { 2001) }\end{array}$} & Low involvement & $\begin{array}{l}\text { Client onsite and considered as a } \\
\text { team member }\end{array}$ \\
\hline & Passive & Active/proactive \\
\hline $\begin{array}{l}\text { Organization culture } \\
\text { (Highsmith, 2002) }\end{array}$ & Command and Control Culture & $\begin{array}{l}\text { Leadership and Collaboration } \\
\text { Culture }\end{array}$ \\
\hline $\begin{array}{l}\text { Market } \\
\text { (Perrin, 2008) }\end{array}$ & Mature/Main Street market & Dynamic/Early market \\
\hline $\begin{array}{l}\text { Measure of success } \\
\text { (Highsmith, 2010) }\end{array}$ & Conformance to plan & Business value delivered \\
\hline
\end{tabular}

Table 2-1: Traditional and agile perspectives on software development 
Traditional, heavy methods draw on documentation, process and formality. They are well adapted for predictability and optimizing activities. Reliance on tacit knowledge is reduced by documentation and formality. On the other hand, light, agile methods rely on understanding, skills, and tacit knowledge. They are well adapted for exploratory activities (Cockburn, 2007) because they allow people to reduce their dependency on documentation, plan, processes and formality (Figure 2-6).

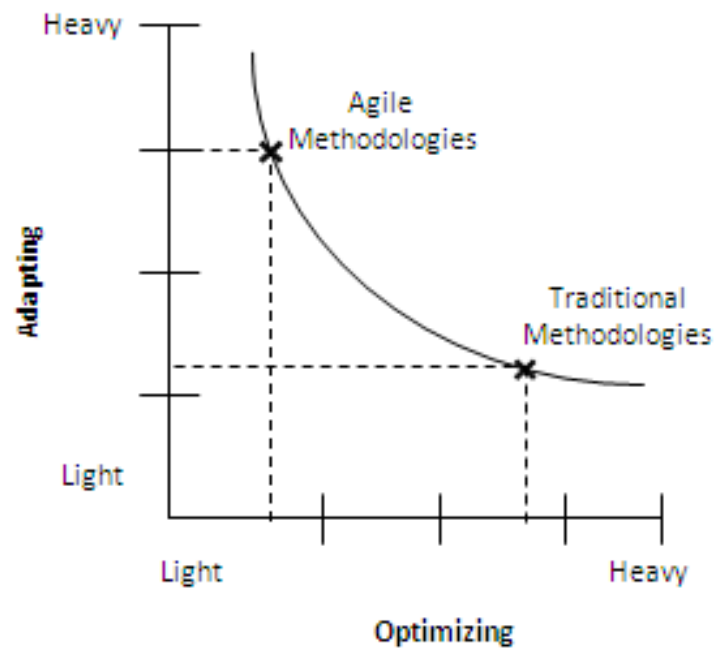

Figure 2-6: Balancing optimizing and adapting dimensions (Adapted from Highsmith, 2002, p. 360)

Traditional methodologies, based on a linear or incremental life cycle, define the software architecture at the beginning of the project, whereas agile projects use the YAGNI principle: "You Aren't Going to Need It". Hence, only features that are needed for the current iteration are designed and implemented. Code that may be needed for future functions is developed only as needed, which may cause, in certain cases, costly reworking of already implemented features. It is therefore potentially risky on large projects.

Traditional projects need specialist team members, assigned to a task and told what to do, when, and how to do it, according to a pre-specified plan. In agile projects, the team of specialists is replaced by a team of co-located generalists who self-organize as needed. The role of the project manager is also different according to the approach chosen. Traditional projects need a project manager who first focuses on mutual agreement to a detailed contract specifying cost and deadline targets, and then organizes the project according to the plan and budget. In this situation, the project 
manager's focus will be on compliance and task completion. On the other hand, the project manager is also a project leader, whose role is to remove obstacles so that the team can get the job done. Here the focus will be on value delivery. The project manager will try to set up a collaborative relationship with the customer (Perrin, 2008; Highsmith, 2010). Highsmith (2010, p. 63) summarizes his role thus: "A traditional project manager focuses on following the plan with minimal changes, whereas an agile leader focuses on adapting successfully to inevitable changes."

In terms of market and culture, traditional methodologies are usually applied in mature markets and work best in companies that have a command and control culture. In contrast, agile methodologies thrive better in dynamic and emergent markets and in a culture characterized by leadership and collaboration (Highsmith, 2002).

Finally, traditional methodologies measure success as conformance to the initial plan. In contrast, agile methodologies are value-driven since they only execute work that will deliver value to the project. Agile methodologies measure project success in terms of the value delivered to the customer.

\subsection{Contingency Approach}

The contingency theory is used in the current study to relate development approaches and project characteristics. Project contingency theory is defined as "the extent of fit or misfit between project characteristics and project management approach" (Sauser et al., 2009, p. 666).

In project management, one size does not fit all (Shenhar, 2001). Thus choosing the right approach for the right project is an important success factor in project management. Not every project requires the same development methodology. Both agile and plan-driven approaches have shortcomings and selecting the right methodology helps reduce cost and risk, meet the project's objectives in terms of schedule and scope, avoid excessive documentation, and improve quality (Charvat, 2003; McConnell, 1996). However, it can be a challenge to determine what approach provides the best fit. There are also some issues with methodology selection. 
Familiarizing the team members with a new methodology will take time (Charvat, 2003, p. 89). In addition, people or organizations tend to be either traditionalist or agilist and thus favour one approach without considering the alternative (Shenhar, 2001).

\subsubsection{Definition of SDM fit}

Wysocki (2009) explains that the best development methodology is based on both the project characteristics and the business and organizational environment in which the project is conducted. Therefore in the current research contingency factors are organized into the two following categories: project and project environment. The second set of factors, which relates the project's relationship with its parent organization (Howell et al., 2010), may have an important influence on the project. For example, Shenhar (2001, p. 395) describes projects as "temporary organizations within organizations". Thus similar projects in different organizations may require different methodologies. From the above reflection, a definition of fit in the context of the current study is as follows:

SDM fit is the choice of a SDM that delivers project success in the context of relevant factors associated with the project and project environment.

To measure the impact of these factors on fit, an objective measure of fit is needed. This is discussed next.

\subsubsection{Measuring SDM fit}

To evaluate the degree of fit or misfit (that is, the level of appropriateness of the methodology for a given project), success criteria are needed to judge the level of success or failure of the methodology (Cooke-Davies, 2002). Indeed, SDM fit is about the choice of the SDM that best helps the successful completion of the project (Perrin, 2008). 
Cockburn (2007) uses the notion of 'methodologically successful projects' to determine projects that have been executed with an appropriate methodology. Although he gives details about the criteria to evaluate methodological success, some of them are difficult to measure and their significance has not been tested. To measure SDM fit, the current study will employ the notion of 'project success'. Indeed, if the project is perceived to be successful, it can reasonably be considered that the SDM used was appropriate. For the rest of this study, it is therefore considered that SDM fit is a necessary condition, though not sufficient condition, of project success

The notion of project success is hard to define and measure because of important differences between notions of success in different project types and industries (Highsmith, 2010; Shenhar, Tishler, Dvir, Lipovetsky, \& Lechler, 2002). Despite much research on project success, there is no universal way to evaluate the success of a project (Shenhar \& Dvir, 2007).

As discussed previously, traditional approaches usually measure success as conformance to scope, schedule, and cost. However, the use of these variables from the iron triangle is often criticized because it is too narrow and does not consider the return on investment, the value delivered to the organization, and commercial success (Jugdev \& Muller, 2005; Mohagheghi, 2008; Shenhar et al., 2002). On the other hand, agile approaches measure success in terms of response to change and value delivered to the customer. Therefore there are no consistent measures available across different project management communities. Moreover, project success may also differ according to the perspective of the assessor and different people will have different opinions on the success of the project (Freeman \& Beale, 1992). In particular, Huisman and Iivari (2006) showed that there is a difference between the Information System (IS) manager and the system developers in the perception of the benefits and problems associated with SDMs.

As Mohagheghi (2008, p. 14) explains, "one common challenge of any evaluation is to choose evaluation criteria". Table 2-2 presents four studies on methodology selection, each of which employ different measures of success to determine fit. Few of these studies included a rationale for the selection of success criteria. 
Shenhar et al. (2002), based on previous research (Dvir \& Shenhar, 1992), consider that project success can be measured by three dimensions: meeting design goals (functionality, time, budget); benefit to the customer; and commercial success and future potential. The first two dimensions were found to be the most important (Lipovetsky, Tishler, Dvir, \& Shenhar, 1997; Papke-Shields, Beise, \& Quan, 2010, p. 658). These dimensions are the most appropriate for measuring project success in the current study as they encapsulate both traditional and agile views.

\begin{tabular}{|c|c|c|}
\hline Study & Measure & Comment \\
\hline Chow and Cao (2008) & $\begin{array}{cl}\text { 'Project Success' } \\
-\quad \text { Quality } \\
-\quad \text { Scope } \\
-\quad \text { Time } \\
-\quad \text { Cost }\end{array}$ & $\begin{array}{l}\text { This study focuses on the } \\
\text { critical success factors in agile } \\
\text { software projects }\end{array}$ \\
\hline $\begin{array}{l}\text { Hardgrave, Wilson and } \\
\text { Eastman (1999) }\end{array}$ & $\begin{array}{l}\text { 'System success' } \\
-\quad \text { User satisfaction }\end{array}$ & $\begin{array}{l}\text { This study focuses on the } \\
\text { success of the prototyping } \\
\text { development methodology }\end{array}$ \\
\hline $\begin{array}{l}\text { Misra, Kumar, and } \\
\text { Kumar (2009) }\end{array}$ & $\begin{array}{ll}\text { 'Success' } \\
-\quad \text { Reduced delivery schedule } \\
-\quad \text { Increased return on } \\
\text { investment } \\
-\quad \text { Increased ability to meet the } \\
\text { current customer } \\
\text { requirement } \\
-\quad \text { Increased flexibility to meet } \\
\text { changing customer } \\
\text { requirements } \\
\text { - Improved business processes }\end{array}$ & $\begin{array}{l}\text { This studies focuses on the } \\
\text { success of agile software } \\
\text { development projects }\end{array}$ \\
\hline $\begin{array}{l}\text { Ratbe, King and Kim } \\
(2000)\end{array}$ & $\begin{array}{c}\text { 'Application System Success' } \\
-\quad \text { User satisfaction } \\
-\quad \text { System utilization }\end{array}$ & $\begin{array}{l}\text { This is a survey of both system } \\
\text { developers and users }\end{array}$ \\
\hline
\end{tabular}

Table 2-2: How previous studies on methodology selection or implementation measured success

Shenhar and Dvir (2007) suggest that an additional dimension has an impact on the development team. This is developers' response to the project and the methodology. Developers have an important role in the successful deployment of a methodology; they may resist a methodology that they regard as not useful (Riemenschneider et al., 2002). This third dimension also appears useful in the current study of SDM fit. 


\subsubsection{Software development methodology fit}

\subsubsection{SDM fit models}

This section reviews the research on SDM fit in order to find "objective" contingency factors, that is, those that predict SDM fit as previously defined. In 1985, Burns and Dennis published a study on the selection of an appropriate development methodology. They compared traditional approaches with iterative prototyping approaches, and presented a contingency approach based on project size and project uncertainty. In 2000, Ratbe et al. also produced research in this area. Their study did not include agile methodologies (agile as a term to describes methodologies was only coined in 2001), but included the prototyping approach. They distinguish three contingency variables: uncertainty, complexity, and the experience of the system user.

Key research that includes both traditional and agile methodologies has been produced by Boehm and Turner (2003, 2004). They build on Cockburn (2002) and other studies to describe a risk-based approach. Methodology selection, engineering and tailoring are based on an assessment of environmental, agility-oriented, and plandriven risk.

The risk associated with an inappropriate choice of project methodology is reduced by first assessing project factors to ascertain how well the project fits with either the agile or the plan-driven approach. Methodology selection is determined by an assessment of five critical factors (need for personnel supervision, criticality, project size, culture, and certainty), which are measured on a scale from pure plan-driven to pure agile (Figure 2-7). They developed these factors from a study of the home ground characteristics of traditional and agile approaches. The collective match on all five project factors determines the profile of the project (typically illustrated by superimposing a radar plot on Figure 2-7). The use of 'critical' factors and a riskbased approach offers insightful guidelines for the project managers who approach software development from within the confines of a single, favourite methodology, and therefore lack an appreciation of how to achieve a successful project. 
Boehm and Turner (2003) support their model by describing projects where the project factors do not favour a pure methodology, and where risks are reduced by including practices associated with the opposite (complementary) methodology. Thus hybrid methodologies are developed. The best-fit methodology may also evolve over time. This is why it is recommended that project development and the methodology initially chosen should be continually monitored (Boehm \& Turner, 2003). However, there are benefits and disadvantages associated with a change in management approach during the project (Wysocki, 2009).

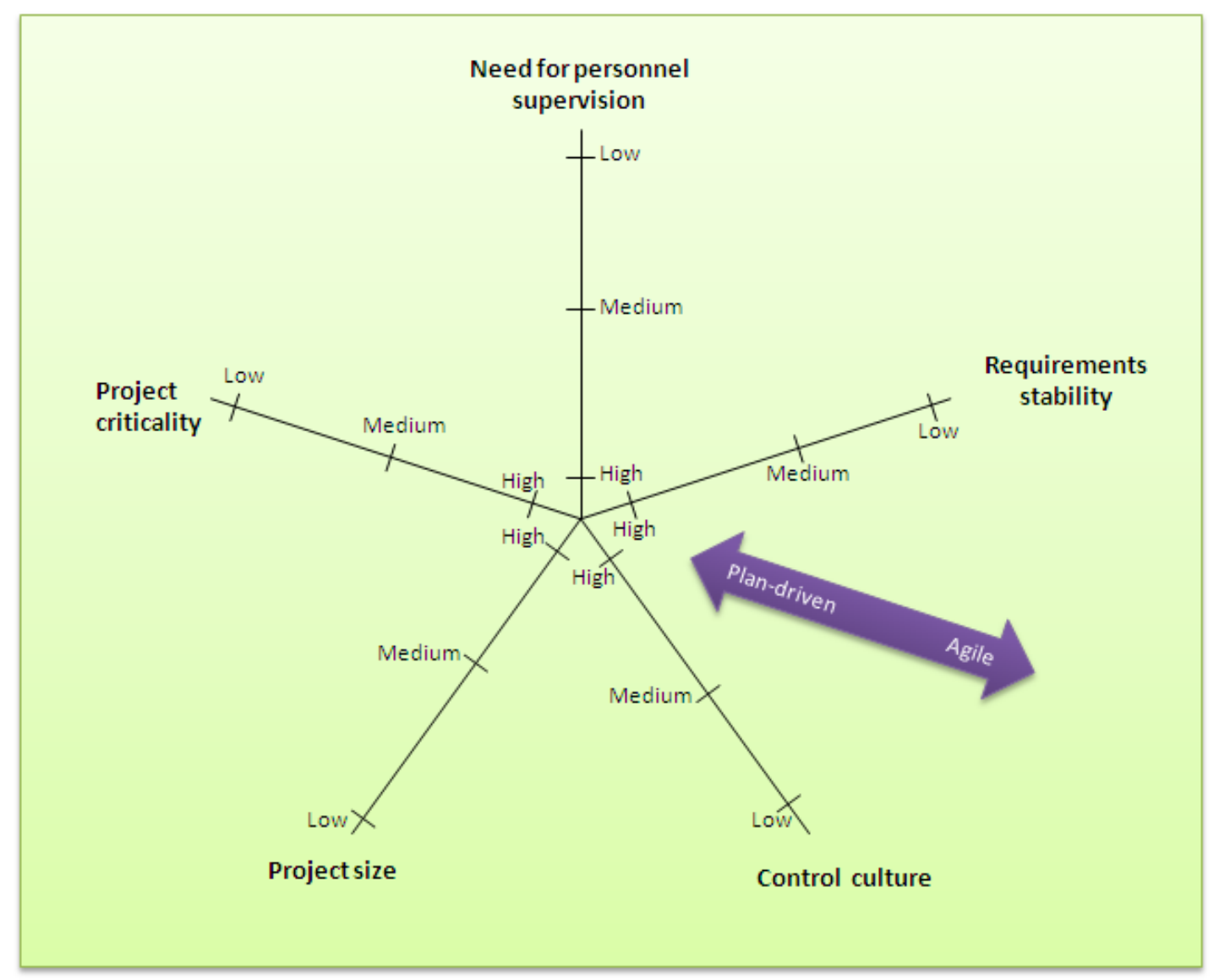

Figure 2-7: Factors that discriminate between agile and plan-driven methodologies (Adapted from Boehm \& Turner, 2003, p. 59)

\subsubsection{Review of Boehm and Turner's contingency factors}

The five project factors described in Boehm and Turner $(2003,2004)$ and presented in Figure 2-7 are further investigated to show the conditions under which the methods are most likely to succeed (Turner \& Boehm, 2003). Reading anti-clockwise 
from the top of Figure 2-7, the factors are: need for personnel supervision, project criticality, project size, control culture, and requirements stability.

Need for personnel supervision

Traditional projects need highly skilled staff at the beginning of the project (during the project definition phase), and then junior or lower-skilled staff can do the assigned work by following pre-established plans. Naturally, highly skilled staff members also perform very well on all phases of traditional projects.

On the other hand, agile projects need a high level of senior and highly skilled people throughout the entire project in order to continuously adapt to change. The talents and skills of individuals are of paramount importance in agile development; individual competency is considered a critical factor in project success (Cockburn \& Highsmith, 2001). Boehm (2002) reminds us, however, that 49.99 percent of the world's software developers are below median and therefore recruiting only the top people may be difficult.

In summary, Boehm and Turner (2003) associate a high-level of personnel supervision with traditional methodologies and a low level of personnel supervision with agile methodologies.

\section{Project criticality}

Project criticality is a factor positively related to the potential damage that the project can cause if there is a defect. Cockburn (2007) divides criticality into four categories defined in terms of the loss caused by defects in the operational product:

1. Loss of comfort where a defect only has a very minor impact

2. Loss of discretionary monies where a defect can be fixed pretty easily because of good backup procedures. According to Cockburn (2007), most projects' criticality is at this level.

3. Loss of essential monies where the company may go bankrupt because of a defect in the system. 
4. Loss of life where defects can be catastrophic, such as in nuclear power stations or flight control systems.

Boehm and Turner (2003) added another category to distinguish between the loss of a single life and the loss of several lives.

When criticality increases, the controls in place should be tighter and the tolerances should decrease. Thus highly critical projects require controls and rigidity that are provided by traditional approaches. On the other hand, these approaches may be too rigid for low criticality projects, which will be better managed by agile methodologies.

\section{Project size}

Boehm and Turner (2003) measure this factor by the number of people working on the project. Charvat (2003) notes that project size and methodology are connected by a positive feedback loop. In other words, with more people, more coordination is needed. Thus a heavier methodology is required, whereas a small project needs a lighter methodology. Highsmith (2002, p. 358) points out that about 60 percent of the world's software projects have 10 or fewer people.

As agile projects rely on tacit knowledge rather than documentation, their scalability is perceived to be limited. For a team of $\mathrm{N}$ members, there are $\mathrm{N}(\mathrm{N}-1) / 2$ communication paths. Thus agile is thought to work best on small projects (fewer than 10 people). In contrast, plan-driven approaches tend to be comprehensive and communication is generally unidirectional from one entity (for example, report or contract) to people. Traditional approaches may find it hard to tailor down to small projects (Boehm \& Turner, 2003). Finding the right balance is therefore important in correctly coordinating the team members without reducing their productivity (Charvat, 2003).

However, the importance of this factor in the choice between agile and traditional methodologies is questionable. Some agilists argue that agile methodologies can be scaled up to accommodate projects comprising many team members while retaining the agile philosophy of the methodology (Highsmith, 2010). Hence a 500-person 
team can be agile because it is based on agile values, even though the level of documentation and coordination has been increased over that associated with a smaller project based on agile values. Nevertheless, Dybå \& Dingsøyr (2009), in a study of 36 research papers on agile, found that agile methodologies are usually not the best choice for large projects. Moreover, Leffingwell (2007) explains that agile methodologies have originally been defined and recommended to small team environments as ready access to, and interaction and collaboration with, customers are the defining rules.

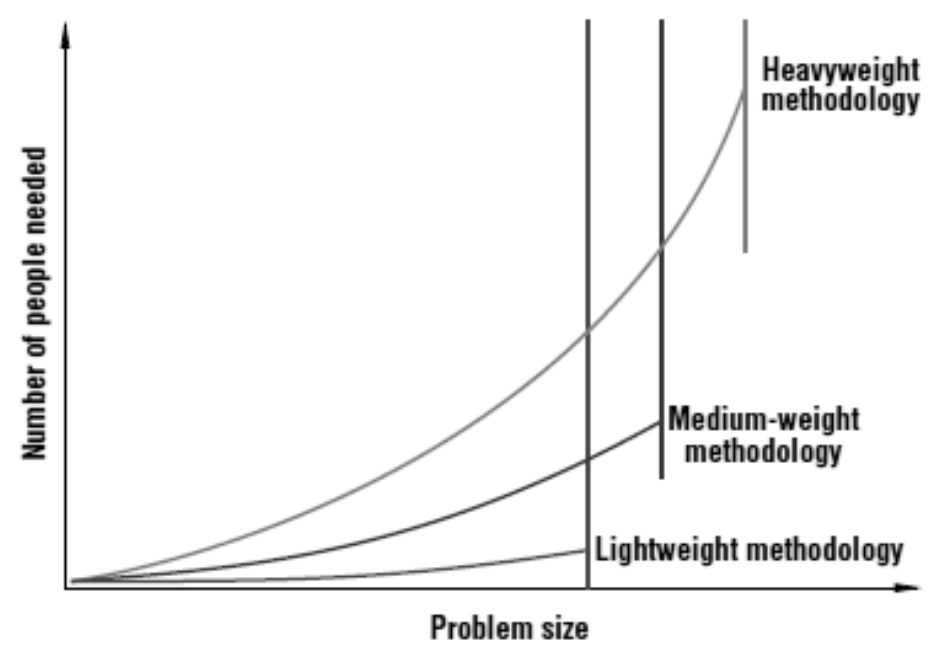

Figure 2-8: Problem size, number of people needed, and methodology choice (Reproduced from Cockburn, 2007, p. 198)

Finally, the graph above (Figure 2-8) shows that there is a limit to the size of the problem that can be solved with a given number of people. Light methodologies cannot solve very big problems. However, they require less people than heavy methodologies on small projects.

\section{Control culture}

Culture is defined by Charvat (2003) as "the personal philosophies of the people involved in these projects". Highsmith (2010) explains that agile methodologies thrive in innovative cultures as in start-up companies. Staff members are empowered and feel comfortable with freedom. On the other hand, traditional methodologies will be more appropriate in well-established companies with a control culture. People in 
these organizations feel comfortable with clear policies and procedures that clearly define their role in the project (Boehm \& Turner, 2003). In Figure 2-7, this axis represents freedom versus order.

Some people tend to be resistant to the prescribed approach of developing software and all project workers are unlikely to feel comfortable with the same approach. Thus if the methodology does not match the culture of the team members, the project is more likely to fail. Turner and Boehm (2003) consider that this factor may be the most significant challenge to the integration of a new SDM in a company. Migration to agile methodologies in companies poses issues, in particular because of the culture in place.

\section{Requirements stability}

The requirements stability component is related to the stability of the requirements provided to the development team. It was originally named by Boehm and Turner (2003) as 'dynamism' and was measured as the number of requirements changes per month. If the requirements change rate is high, then agile methodologies are recommended since they have been specifically developed to address the problems of rapid change. Iterative delivery helps reduce uncertainty and leads the project through uncertainty (Pixton et al., 2009). Traditional approaches plan for every task in advance and many of them will not be executed because of numerous changes. As a consequence, time is wasted if the plan needs to be reworked. Agile methodologies avoid that issue by just-in-time planning.

In the case of a low requirements change rate, the traditional approach and its big, up-front design work best. As there is no or little change during the project, the plan does not need to be modified and can thus be optimized. Future features are prepared in the design and all the pieces are designed to fit well together. Agile methodologies may also work on projects that have a low level of change. There is, however, in that case, a lack of optimization and initial planning that can slow down the project. The principle of simplicity may lead to potentially expensive reworking because some features are not prepared early in the design, therefore causing reworking and a waste of time. 


\subsubsection{Current research in SDM fit}

Three recent research publications that build on the above stream of research on SDM fit (Highsmith, 2010; Pixton et al., 2009; Wysocki, 2009) are briefly reviewed.

Highsmith (2010) consolidates the critical factors in SDM fit into three categories (Table 2-3). The project factors (complexity and uncertainty) should dominate the choice of the methodology (Highsmith, 2010). Managing uncertainty is best done with agile and flexible practice, while complexity requires structure. Further, Pixton et al. (2009) argue that understanding these two key factors (uncertainty and complexity) help indentify better ways to manage the project. Wysocki shows graphically (Figure 2-9) what the best development life cycles are according to the level of complexity and uncertainty. Several previous researchers also based their contingency theory on uncertainty and complexity (Howell, Windahl, \& Seidel, 2010; Little, 2005; Shenhar, 2001).

\begin{tabular}{ll}
\hline Critical elements in SDM fit & Their main components \\
\hline Project factors & Complexity (problems associated with managing multiple, \\
& complicated but tractable issues): team size, team \\
& distribution, mission criticality, and domain knowledge gaps. \\
& Complexity requires structure and discipline \\
& Uncertainty (problems associated with making sense of \\
& dynamic situations): market uncertainty, technical \\
& uncertainty, and project duration. Uncertainty requires \\
& agility and flexibility \\
& Structured, conformance-to-plan cultures. \\
Cultural factors & Thriving on order \\
& versus \\
Agile, flexible, collaborative cultures. \\
Thriving on chaos \\
Even if this is to be taken into consideration in the choice of a \\
methodology, this should not be the primary factor. In fact, \\
once a methodology is chosen, it can generally be tailored \\
for compliance.
\end{tabular}

Table 2-3: Contingency factors in SDM fit (Adapted from Highsmith, 2010)

Highsmith (2010) argues that project cultural factors are second most important. We note that the cultural factors defined by Highsmith (2010) are not those from the general enterprise environment. Instead they are specifically implicated in software development methodological choice and are described in terms similar to the 
definition of the 'culture' that constitutes one of the five critical project factors defined by Boehm and Turner (2003).

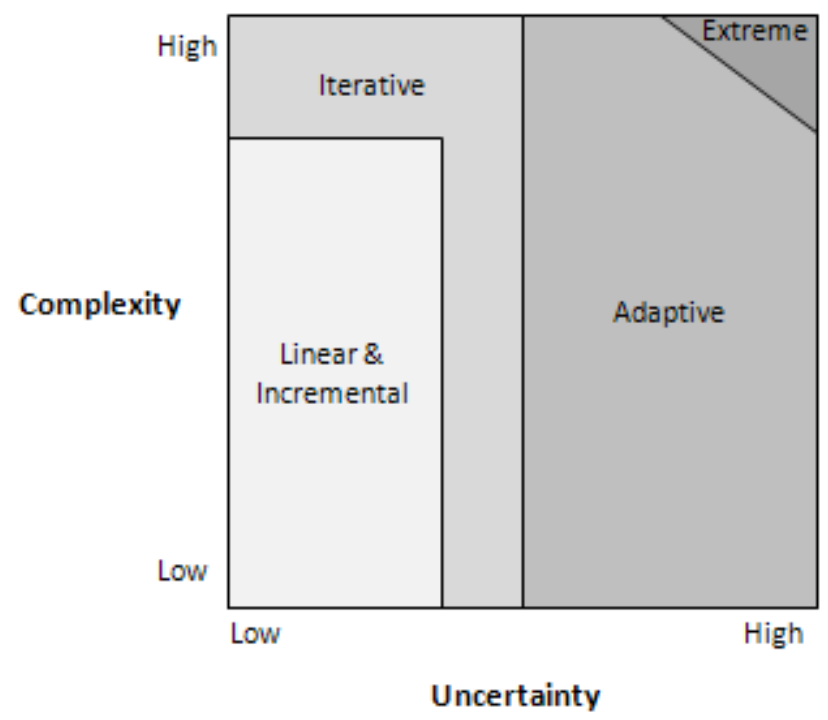

Figure 2-9: The contemporary software development landscape (Reproduced from Wysocki, 2006, p. 37)

\subsubsection{Summary of contingency factors}

In summary, all three of these recent publications emphasize the role played by two project factors - complexity and uncertainty - in determining the best-fit methodology. Complexity - a factor not directly included in the five Boehm and Turner (2003) critical factors - is arguably present through its project size and project criticality components. Uncertainty - making sense of dynamic situations - is related to previous research on dynamism or requirements stability. In particular, Wysocki (2009) argues that the presence of uncertainty is the most important factor although not the only one in determining the need for agile project management.

In addition to these studies on SDM fit, many studies have been published on the success factors of agile methodologies. Indeed, the recent rise in popularity of agile SDMs has attracted the attention of many researchers. Dybå and Dingsøyr (2008) identified 1,996 studies on agile software development published up to 2005. The factors identified in these studies are relevant in the sense that they show under which conditions agile methodologies succeed, and therefore, fit a given project. 
In the table below, 8 project environment and 13 project factors identified by studies on SDM fit and agile methodologies are summarized. The uncertainty and complexity concepts discussed previously do not directly appear in this table, but are present through their constituent factors (like team size and project criticality for the complexity concept, for example). Some factors (such as organization size) are likely to directly or indirectly affect other factors. SDM fit literature is contiguous with the project success literature, and the SDM literature, including Agile which has recently attracted a large number of articles (Misra et al. 2009). It is not possible to identify all the contingency factors identified in the SDM fit literature because the SDM literature lacks clear boundaries, and because cause and effect are frequently indirect. Conversely variables in SDM fit papers are often studied for purposes other than SDM fit. In the interest of both focus and parsimony a relatively limited number of the contingency factors in SDM fit are listed in Table 2-4. There is no agreement on the relative importance of these 21 items, nor how to measure them, or project success.

\begin{tabular}{|c|c|}
\hline Factor & Literature \\
\hline \multicolumn{2}{|l|}{ Project Environment Factors } \\
\hline $\begin{array}{l}\text { Compliance and governance } \\
\text { factors }\end{array}$ & Highsmith (2010) \\
\hline Corporate culture & Misra et al. (2009) \\
\hline Market uncertainty & Highsmith (2010); Pixton et al. (2009); Wysocki (2009) \\
\hline National culture & Misra et al. (2009) \\
\hline Nature of the contract & Koch (2005) \\
\hline Organization size & Charvat (2003); Cockburn (2007); Highsmith (2010) \\
\hline $\begin{array}{l}\text { Top management support for one } \\
\text { approach }\end{array}$ & Ratbe et al. (2000) \\
\hline Training/collaborative learning & Charvat (2003); Livermore (2008); Misra et al. (2009); \\
\hline \multicolumn{2}{|l|}{ Project Factors } \\
\hline $\begin{array}{l}\text { Co-location of the project team } \\
\text { members }\end{array}$ & $\begin{array}{l}\text { Cockburn (2000); Highsmith (2010); Koch (2005); Pixton et al. } \\
\text { (2009); Wysocki (2009) }\end{array}$ \\
\hline $\begin{array}{l}\text { Culture of the project team / } \\
\text { Empowerment through } \\
\text { internalized/qualitative controls }\end{array}$ & $\begin{array}{l}\text { Boehm and Turner (2003); Highsmith (2010); Koch (2005); } \\
\text { Misra et al. (2009); Strode, Huff, and Tretiakov (2009) }\end{array}$ \\
\hline $\begin{array}{l}\text { Customer commitment, } \\
\text { collaboration, and involvement }\end{array}$ & $\begin{array}{l}\text { Ceschi, Sillitti, Succi, and De Panfilis (2005); Chow and Cao } \\
\text { (2008); Koch (2005); Misra et al. (2009); Wysocki (2009) }\end{array}$ \\
\hline $\begin{array}{l}\text { Personnel skills, and team } \\
\text { maturity }\end{array}$ & $\begin{array}{l}\text { Boehm and Turner (2003); Chow and Cao (2008); Koch } \\
\text { (2005); Misra et al. (2009); Pixton et al. (2009); Ratbe et al. } \\
\text { (2000); Wysocki (2009) }\end{array}$ \\
\hline
\end{tabular}




\begin{tabular}{ll}
\hline Factor & Literature \\
\hline Project cost & Charvat (2003); Ratbe et al. (2000); Wysocki (2009) \\
\hline Project criticality & $\begin{array}{l}\text { Boehm and Turner (2003); Charvat (2003); Cockburn (2000, } \\
\text { 2007); Highsmith (2010); Koch (2005); Pixton et al. (2009) }\end{array}$ \\
\hline Project duration & $\begin{array}{l}\text { Highsmith (2010); Pixton et al. (2009); Ratbe et al. (2000); } \\
\text { Wysocki (2009) }\end{array}$ \\
\hline Project size (man hours) & Burns and Dennis (1985) \\
\hline $\begin{array}{l}\text { Project uncertainty / } \\
\text { Requirements stability }\end{array}$ & $\begin{array}{l}\text { Boehm and Turner (2003); Burns and Dennis (1985); } \\
\text { Highsmith (2010); Koch (2005); Pixton et al. (2009); Ratbe et } \\
\text { al. (2000); Shenhar (2001); Wysocki (2009) }\end{array}$ \\
\hline $\begin{array}{l}\text { Proportion of the organization } \\
\text { affected }\end{array}$ & $\begin{array}{l}\text { Ratbe et al. (2000); Wysocki (2009) } \\
\text { Team Size }\end{array}$ \\
\hline Boehm and Turner (2003); Charvat (2003); Cockburn (2000, \\
\hline 2007); Highsmith (2010); Koch (2005); Pixton et al. (2009)
\end{tabular}

Table 2-4: Summary of contingency factors identified in the literature

\subsubsection{Summary}

Few models have been developed for choosing between agile and traditional SDMs. In this context, 'model' means:

The selection, measurement and prioritization of the issues (Table 2-1) and contingency factors (Table 2-4) so as to identify critical factors in SDM fit.

Boehm and Turner's model (2003) is the only academic model identified in this area. Other models have been developed by a practitioner (Little, 2005) or agile proponents (Cockburn, 2000; Highsmith, 2010). Yet no model has emerged as generally accepted and there is a lack of empirical evidence (Howell et al., 2010; Koch, 2005). Moreover, these models prioritize project factors over project environment factors. 


\subsection{Conclusion}

\subsubsection{Research gaps}

The review of SDMs, SDM fit and project success yielded two conclusions.

The first conclusion, that one size does not fit all project environments, is reflected in diverse studies on methodology selection and fit. The degree of alignment between various Project Environment factors and SDM is expected to influence both SDM fit and project success. The second conclusion, that one size does not fit all projects, is reflected in the research by Boehm and Turner (2003) on SDM fit. To achieve SDM fit and Project Success, Project factors must also be investigated during methodology selection.

An analysis of previous research revealed that critical factors in methodology selection and fit have been identified through experience and case study research, but no agreement has been reached. The purpose for the research reviewed above is primarily exploratory and descriptive, rather than analytical or predictive (Collis \& Hussey, 2003, p.10).

Some of the research summarized in Table 2-4 develops operational definitions and related numerical measures. However, measures of the concepts of Project Environment factors and Project factors; measures of SDM on a scale from pure plan-driven to pure agile; and measures of Project Success have not been expressed in numerical form and deployed together as the basis of quantitative research on methodology selection and fit. As a consequence, the research stream, although mature, retains a qualitative and descriptive flavour that eschews quantitative measures and predictive models. A quantitative model that employs quantitative measures of these concepts, and seeks to determine the strength of the relationships among them, has yet to be proposed and investigated. Empirical research based on such a model may reduce at least some of the many things we do not yet know about SDM selection and project success. The current research targets the five research gaps listed in Table 2-5. 
Gap \#1. The role of Project Environment in determining a SDM that fits

Gap \#2. The role of Project Factors in determining a SDM that fits

Gap \#3. The role of Project Environment in determining Project Success

Gap \#4. The role of SDM fit in determining Project Success

Gap \#5. The role of Project Factors in determining Project Success

Table 2-5: Main research gaps

\subsubsection{Description of the research model}

Based on the literature review and the gaps identified above, a model in two parts is proposed (Figure 2-10 \& Figure 2-11). In this model, the relationships (H1 to H5) correspond to the five gaps identified in Table 2-5.

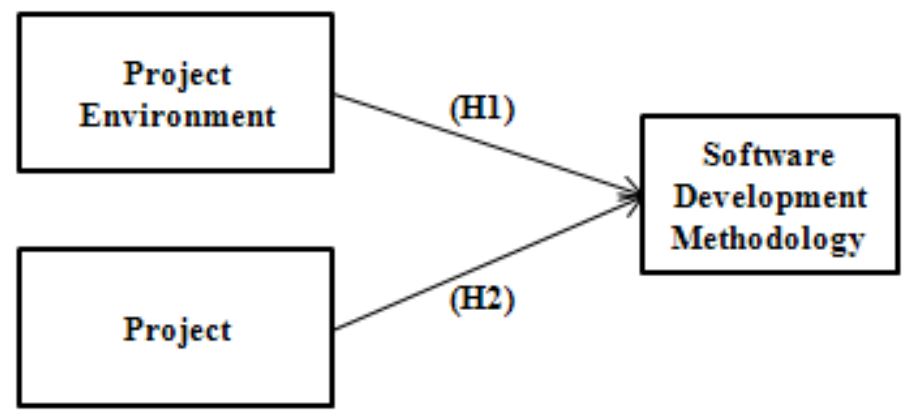

Figure 2-10: SDM fit research model

The first part of the model (Figure 2-10) represents the effect of Project and Project Environment in determining a $S D M$ that fits. This model can be evaluated by studying projects that have achieved SDM fit.

Then by applying this model of SDM fit to all the projects, a measure of SDM fit can be obtained for each of them. This measure of fit constitutes one of the independent variables used in the second part of the model, which evaluates the impact of Project Environment, SDM fit and Project, on Project Success (Figure 2-11).

The purpose of the conceptual development of this model, and its empirical evaluation is to develop a theory of SDM fit that can be tested quantitatively via a survey of a sample of project workers involved in software development. 


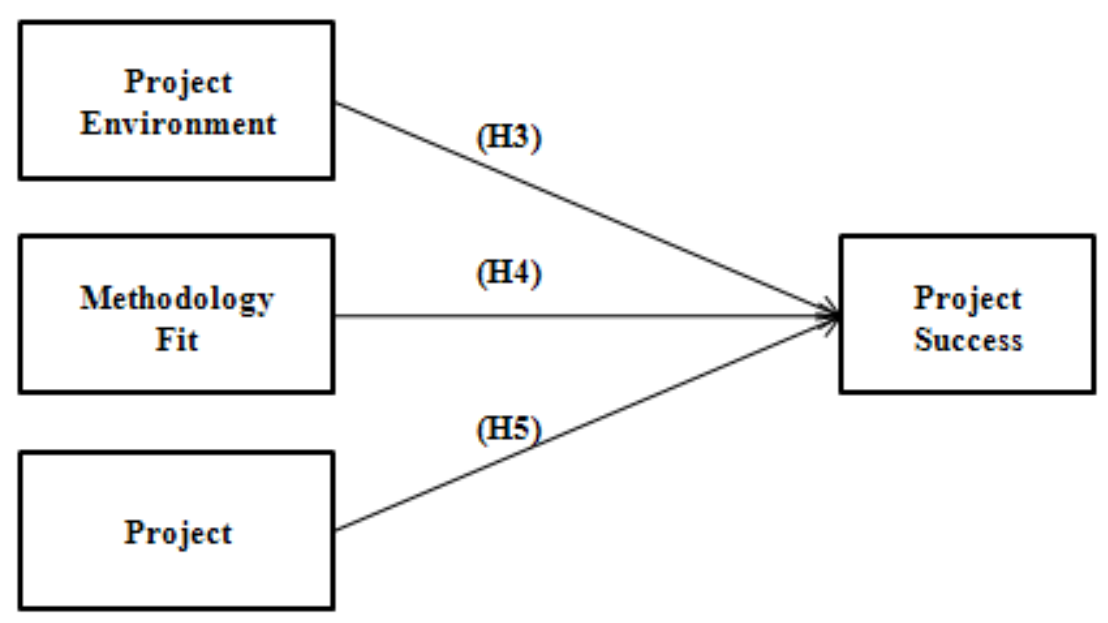

Figure 2-11: Project success research model

The study first identifies the most important factors associated with the Project Environment and Project in predicting the SDM that achieves SDM fit. Following this, the degree to which these factors and SDM fit predict the degree of Project Success achieved is measured.

\subsubsection{Hypotheses}

Firstly, this research aims at determining the critical contingency factors in SDM fit (links H1 and H2 in the research model). SDM fit was defined earlier (p. 23) as the choice of a methodology that delivers project success in the context of relevant factors associated with the project and project environment. Thus our hypotheses for the first part of the model are the following:

\section{H1: Project environment factors influence the choice of a SDM that fits}

H2: Project factors influence the choice of a SDM that fits.

Secondly, this research aims at determining to what extent project environment factors, SDM fit and project factors impact project success (links H3, H4, and H5 in the research model). The hypotheses are the following:

H3: Project environment factors influence project success

H4: SDM fit influences project success

H5: Project factors influence project success. 


\subsubsection{Summary}

This chapter distinguished traditional from agile SDMs. A comparison of these methodologies was presented in Table 2-1. Then SDM fit was defined as the choice of a methodology that delivers project success in the context of relevant factors associated with the project and project environment. Several models of SDM fit, including Boehm and Turner (2003), were reviewed and contingency factors mentioned in the literature were summarized (Table 2-4). This table clearly shows that there is no agreement among researchers on these factors and statistical evidence for particular factors is lacking. Finally, research gaps were highlighted and a research model was developed to address these gaps. 


\section{CHAPTER 3: METHODOLOGY}

This chapter starts by providing operational definitions for the key constructs that are included in the research model. It then informs the reader about the research philosophy and methodology used to gather empirical data to test the model. It also justifies the instruments chosen for data collection. Finally, ethical considerations, credibility and generalization issues are addressed.

\subsection{Operationalization of the Research Model}

\subsubsection{Description of the five clusters}

The variables from the five clusters presented in the research model (Figure 2-10 \& Figure 2-11) are described and defined below.

\subsubsection{Independent variable cluster 1: Project Environment}

Project Environment includes variables that are not specific to the project but that are related to the external environment of the project. The current study considers that the project context may have an impact on the choice of a SDM. As there is no proven and tested list of project environment factors, a list of variables and their operational definitions has been developed (Table 3-1) from issues (Table 2-1) and the seven project environment contingency factors (Table 2-4) identified in the literature review, feedback from a related conference publication (Sheffield \& Lemétayer, 2010), and the researcher's informed intuition. Face-to-face interviews with project workers helped identify the most important of these variables. The selected variables were then measured on a 5-point Likert scale and organized in factors by exploratory factor analysis. 


\begin{tabular}{|c|c|}
\hline Variable & Operational definition \\
\hline \multicolumn{2}{|l|}{ External environment variables } \\
\hline Economic sector & $\begin{array}{l}\text { Client organization is part of government vs. private } \\
\text { sector }\end{array}$ \\
\hline Governance and compliance factors & $\begin{array}{l}\text { Compliance to regulatory requirements or contractual } \\
\text { obligations }\end{array}$ \\
\hline Market uncertainty & $\begin{array}{l}\text { The environment within which the organization operates } \\
\text { is stable vs. unstable }\end{array}$ \\
\hline Power distance & $\begin{array}{l}\text { Power relations \& national culture - Acceptance of large } \\
\text { vs. small power differentials (tall vs. flat hierarchy) }\end{array}$ \\
\hline \multicolumn{2}{|l|}{ Organizational variables } \\
\hline Level of entrepreneurship & Conservative vs. entrepreneurial company \\
\hline $\begin{array}{l}\text { Methodology supported by top } \\
\text { management }\end{array}$ & $\begin{array}{l}\text { SDM supported by top management (plan-driven vs. } \\
\text { agile) }\end{array}$ \\
\hline Nature of the contract & Fixed price vs. flexible/incremental budgeting \\
\hline Size of the organization & $\begin{array}{l}\text { Size of the organization in which the project was } \\
\text { conducted (big company vs. small company) }\end{array}$ \\
\hline \multicolumn{2}{|l|}{ People/community asset variables } \\
\hline $\begin{array}{l}\text { Project manager's certifications or } \\
\text { training }\end{array}$ & $\begin{array}{l}\text { The highest certifications and training the project } \\
\text { manager has in this methodology. }\end{array}$ \\
\hline $\begin{array}{l}\text { Project manager's experience with one } \\
\text { methodology }\end{array}$ & $\begin{array}{l}\text { The methodology with which the project manager has } \\
\text { most experience }\end{array}$ \\
\hline Project workers' certifications or training & $\begin{array}{l}\text { The highest certifications and training the project } \\
\text { workers have in one methodology }\end{array}$ \\
\hline $\begin{array}{l}\text { Project workers' experience with one } \\
\text { methodology }\end{array}$ & $\begin{array}{l}\text { The methodology with which project workers have most } \\
\text { experience }\end{array}$ \\
\hline
\end{tabular}

Table 3-1: Project environment variables

\subsubsection{Independent variable cluster 2: Project}

These variables are related to the characteristics of the project itself. As shown in the previous chapter, there is no full and final agreement on what these variables actually are. A list of all the project variables and their operational definition was developed (Table 3-2) based on Boehm and Turner (2003), the 13 project contingencies identified in the literature, feedback from conference attendees and the researcher's informed intuition. Project variables in Table 2-4 were listed in alphabetical order, as they were derived from many different schemes. In the following, project variables are organized according to five dimensions of a particular scheme, that popularized by Boehm and Turner (2003). In Figure 2-7, reading anti-clockwise from the top, 
these factors are: need for personnel supervision, project criticality, project size, control culture, and requirements stability. Interviews with project workers were conducted to select the most important variables to be measured in the survey. The selected variables were measured on a 5-point Likert scale.

Variable

Operational definition (and link to Boehm \& Turner, 2003)

\section{Boehm and Turner (2003) Category 1. Personnel: (low-> plan-driven)}

Education level of the team members

Experience level of the team
The level of education of the team members (this reduces need for supervision)

Experience of the project team members (this reduces need for supervision)

\section{Boehm and Turner (2003) Category 2. Project Criticality: (high -> plan-driven)}

Impact of failure to deliver the product

Impact of lack of timely delivery (Urgency)

Project criticality
The level to which an inability to deliver the product/software at all would impact the client members (this increases criticality)

The level to which an inability to deliver the product/software on time (within the window of opportunity) would impact the client members (this increases criticality)

The level to which a defect in the product/software would impact the client members (this increases criticality)

\section{Boehm and Turner (2003) Category 3. People Management (high->plan-driven)}

Co-location of the project team members

Project cost

Project duration

Project size

Proportion of the organization affected

Team size
Project team members work at the same site vs. Project team members work in different sites

Total cost of the project (this increases project size)

The duration of the project in man months (this increases project size)

Estimation of the total amount of uninterrupted labour required to complete the project (man years) (this increases project size)

Proportion of the organization affected by the new product / software (linked via organization size to the number of stakeholders) (this increases project size)

Peak number of project team members working on the project (this increases project size)

Boehm and Turner (2003) Category 4. Culture of the project team (low->plan-driven)

Customer adaptability
Flexibility of the customer in adopting different methodologies policies (increased flexibility increases opportunity for procedural empowerment and decreases control culture) 


\section{Variable}

Operational definition (and link to Boehm \& Turner, 2003)

Customer commitment

The level of engagement / collaboration the customer is willing to put in the project policies (a higher level increases opportunity for procedural empowerment and decreases control culture)

Procedural empowerment determined roles and following fixed policies (if so, this determined roles and following fixed policies (if so, this reduces procedural empowerment and increases control culture)

Boehm and Turner (2003) Category 5. Dynamism: (low->plan-driven)

Project uncertainty

Technological uncertainty
The user requirements are unstable (this reduces requirements stability)

The technology used is well known by the project team vs. it is totally new

Table 3-2: Project variables

\subsubsection{Dependent variable 1: SDM}

These variables determine what approach has been selected on a planning spectrum from pure plan-driven (Adaptive) to pure agile (Prescriptive). These variables come from the agile manifesto (Figure 2-3), which clearly contrasts the two categories of methodologies. They were measured on a 5-point Likert scale from pure plan-driven (Strongly disagree) to pure agile (Strongly agree). In addition to these variables, an extra question evaluated the life cycle used on the project in terms of the concepts presented in sections 2.2.2 and 2.3.2 (linear, incremental, iterative or adaptive) to add assurance that the SDM is correctly measured on the spectrum from pure plan-driven to pure agile (Table 3-3).

\begin{tabular}{|c|c|}
\hline Individuals over Processes & $\begin{array}{l}\text { Individuals and interactions were valued more than processes and } \\
\text { tools }\end{array}$ \\
\hline $\begin{array}{l}\text { Working code over } \\
\text { documentation }\end{array}$ & $\begin{array}{l}\text { Working software was valued more than comprehensive } \\
\text { documentation }\end{array}$ \\
\hline Collaboration over contract & $\begin{array}{l}\text { Close customer collaboration was valued more than strict } \\
\text { adherence to a predetermined contract }\end{array}$ \\
\hline Change over plan & $\begin{array}{l}\text { Initiating and responding to change was valued more than strict } \\
\text { adherence to a predetermined plan }\end{array}$ \\
\hline Development life cycle & $\begin{array}{l}\text { Software development life cycle (linear, incremental or } \\
\text { iterative/adaptive) }\end{array}$ \\
\hline
\end{tabular}

Table 3-3: SDM variables 


\subsubsection{Intermediate variable: SDM fit}

In contrast to all the other variables in the model, SDM fit is a latent variable that was derived from a statistical model rather than directly measured by the survey. This measure was based on the difference between what the SDM fit Model predicted and the actual methodology used. A low difference implied a high SDM fit and vice versa.

\subsubsection{Dependent variable 2: Project Success}

Finally, this cluster of variables measures the project success based on Dvir and Shenhar (1992), Papke-Shields et al. (2010), Shenhar et al. (2002), Shenhar and Dvir (2007). All these variables are measured on a 5-point Likert scale from strongly disagree to strongly agree.

\begin{tabular}{ll}
\hline \multicolumn{1}{c}{ Meeting design goal } & \\
\hline Time & The project was completed on time or earlier \\
\hline Budget & The project was completed within or below budget \\
\hline Functionality & The project met the customer's requirement \\
\hline Quality & The project delivered a good working product \\
\hline \multicolumn{1}{c}{ Benefits to customer } & The product addresses a recognized need \\
\hline Addresses a need & The product is used by the customer \\
\hline Product is used & The product satisfied the customer \\
\hline Customer is satisfied & \\
\hline Impact on the project team & The project team was highly satisfied \\
\hline Team is satisfied & The team would work the same way again \\
\hline Team would work the same way again &
\end{tabular}

Table 3-4: Project success variables

\subsection{Research Philosophy}

This research project was conducted from a positivist perspective based on the ontological assumption that reality is external and objective (Easterby-Smith, Thorpe, \& Jackson, 2008). This perspective is appropriate when the research aims to describe a situation through observation and measurement (O'Leary, 2004). In this 
research, the causal relationships between independent variables (contingency factors) and the selected SDM is investigated to explain SDM fit and project success. This study is hypothesis-driven and empirical. In positivist studies, it is important to ensure that the data gathered is as objective as possible and accurately represents the phenomenon observed. Positivist research findings are not biased by the researcher and his beliefs, as the researcher is independent of the situation being studied. Reliability and reproducibility are important in positivist research. Reliability and validity issues are specifically addressed at the end of this chapter. As in many positivist studies, a quantitative approach was chosen and data from a large sample was collected in order to obtain statistically significant findings that can be generalized to the population studied.

\subsection{Research Instruments}

To fill in the research gaps and test the research model, the main research instrument was a web-based survey. But first, semi-structured interviews were conducted in order to select the most important constructs for the two independent variable clusters and improve the validity of the survey.

The author and his supervisor wrote a conference paper (Sheffield \& Lemétayer, 2010). This paper presents an initial overview of the literature on the topic of SDM fit as well as the research model. This paper was presented at the Project Management Institute (PMI) conference in Melbourne, Australia (22-24 February 2010). The researcher sought feedback and participants for the survey. The paper generated discussion amongst the 60 people who attended the session. During the conference, 125 names and email addresses for the survey were collected, as well as some valuable feedback, which enabled the researcher to refine the constructs before the beginning of the interviews. 


\subsubsection{Semi-structured interviews}

\subsubsection{Card sort technique}

These interviews allowed a conceptual exploration of the variables affecting SDM fit. First, the goal was to ensure that the constructs that would be measured in the survey were aligned with the reality of IT project management in organizations. Second, interviews were intended to improve the validity of the questionnaire by talking to professionals in IT project management. Interviews focused on the Project and Project Environment clusters as those were the clusters in which there was the most uncertainty regarding the concepts that should have been included.

A card sort technique was used to collect data from interviewees (Faiks \& Hyland, 2000). On each card, a concept was written (i.e. a variable that may affect SDM fit) that needed to be organized by the respondent into various categories. The card sort technique delivered a rank ordered list of candidate variables.

This approach is a reliable and inexpensive method to identify trends and patterns that emerge from the opinions of diverse IT professionals (Rugg \& McGeorge, 1997). For example, this technique is often used by information architects in the design of websites because it reveals users' mental models (Faiks \& Hyland, 2000). Other advantages of the card sort technique are that it is simple and quick to execute (Spencer \& Warfel, 2004). One limitation, on the other hand, is that card sorting implies a clear-cut distinction between adjacent categories, while in reality these boundaries are generally fuzzy (Rugg \& McGeorge, 1997).

\subsubsection{Implementation}

The twelve project environment variables (Table 3-1) and sixteen project variables (Table 3-2) selected in the research model were each written on a card. On the back of the card, an operational definition was provided so as to assist accurate interpretation of the variables by the interviewees. For recording purposes, all the cards were randomly numbered. Cards were separated in two sets: Project Environment factors and Project variables. Participants were given cards from each 
set in a random order and had time to go through each of them before the beginning of the sorting procedure. All the interviews were face-to-face and each participant was interviewed separately. The length of each interview was between 30 and 60 minutes. A snowball sampling technique was used to find participants.

There are two primary methods for performing card sorts: open card sorting and closed card sorting. In the first case, participants sort cards into groups that they feel appropriate. In the second case, participants are asked to sort cards into pre-specified groups (Spencer \& Warfel, 2004). The latter method was used in this study so as to ensure the homogeneity of the data, and thus allow comparison between participants.

Interviewees were first asked to sort the given set of cards, based on their own experience, into two piles, one being most important variables, and the other being less important variables in methodology selection. They were prompted to do so by the following question: "What variables do you think have an impact on the selection of the SDM for a given project?"

Because our list of variables may not be exhaustive, blank cards were made available and participants were offered the opportunity to add new variables. Next, interviewees were asked to rank the important variables' pile in groups ranked by importance. As participants sorted the cards, the researcher asked them to comment on their choices in order to get a better understanding of the reality of methodology selection. This procedure was executed firstly with the project environment variables and secondly with the project variables.

The recording of the sorting session was facilitated by the unique number written on each card. A form prepared in advance (Appendix C) was filled in quickly by the researcher to record the ranking choices. Moreover, the card numbering system helped when making notes of comments about specific variables. Most participants illustrated their choices by examples from their experience. Some of their comments are reported confidentially in this report.

At the end of the interview, participants were presented with a copy of our conference paper (Sheffield \& Lemétayer, 2010) to give them an explanation of this research study and present them with the research model, on which they were welcome to give feedback. 
After each interview, an email was sent to the interviewee to thank him/her for participating in the study. This was also the opportunity to ask for further contacts as part of the snowball sampling process.

\subsubsection{Data analysis}

The role of the data analysis was to determine what variables were perceived as having the most influence in methodology selection. This was a way to ensure that the right constructs would be measured in the upcoming survey.

This analysis was conducted using an Excel spreadsheet to determine the cards that were the most often selected. Each variable was given voting points according to its ranking by the participants. Variables selected as most important got five points. The second most important variables got four points, and so forth. Variables that were not selected got zero points. For each variable, the voting points from all interviews were then added up and the variables were ranked by their total number of points. This gives an accurate snapshot of the data, and the variables most often cited. The analysis of the data gathered from the interviews is reported in the next chapter.

\subsubsection{Ethical considerations}

Human Ethics Committee approval was obtained from Victoria University of Wellington before the interviews were conducted. Each interviewee received an information sheet (see Appendix A) and was asked to sign a research agreement (see Appendix B) ensuring the confidentiality of the interview and that the reporting would be in non-attributable form. Participants had the right to withdraw at any time prior the beginning of data analysis, but nobody asked to withdraw. Questions asked were not sensitive and it was not intended to collect strategic or confidential information from interviewees. Rather, the researcher tried to elicit how methodologies are selected in practice and what variables are the most important from the interviewee's own experience.

The findings in this report and other publications are only presented in an aggregated, non-identifiable form. Thus from the findings of this report, it is not 
possible to identify any individual or organization. Interviews were neither audiorecorded nor transcribed. Some notes of the key comments from interviewees were taken during the interviews. They are reported in a confidential form in this report to illustrate some of the key findings. Notes and data were kept in locked files accessible only to the researcher and his supervisor. All this material will be destroyed two years after the end of the study. The delay is to allow verification of the data for latter publications in academic journals.

\subsubsection{Resources}

The amount of resources needed to conduct the interviews was fairly low. All the interviews took place in Wellington. Some of them were conducted at Victoria University and others in cafés. In the latter situation, interviewees were offered a drink. Interview analysis was conducted with Microsoft Excel, which is available on university computers.

\subsubsection{Survey}

\subsubsection{Description}

This study aims at evaluating 1) the critical factors in matching SDM to variables in the Project and Project Environment and 2) the role of these factors and SDM fit in determining Project Success. To achieve this, an online survey was conducted. The advantage of a web-based survey is that it is convenient for software project managers who have easy access to a computer and the internet. Moreover, it prevents transcription errors and allows reaching a large number of participants all around the world in a very short period of time. Finally, it ensures anonymity as the respondent does not supply identifying information such as an email address.

The targeted participants were project workers involved in software development projects. A non-probability sampling method was used for this research as it was not possible to randomly select practitioners from the population being studied. In particular, a quota sampling technique was used to make sure that both agile and 
non-agile projects were approximately equally represented. To achieve this, the potential respondents were not all contacted at the same time. Respondents were contacted progressively according to the kind of data needed.

Several channels were employed to refine the sample and obtain respondents. It was intended to obtain a sample of at least one hundred participants. The people contacted to participate in the survey were:

- A group of 125 project workers who volunteered to participate in the survey at the PMI conference in Melbourne

- A group of 229 project workers, who became signatories of the agile manifesto in 2009 or 2010 . They were contacted directly from the contact details they left on the agile manifesto website

- 126 agile user groups throughout the world

- 8 Java, .NET, or PRINCE2 user groups

- The members of the Australian Institute of Project Management.

The targeted respondents were contacted by email only once. As the survey was anonymous, it was impossible to know who had responded and who had not. Thus targeted reminders were not sent. The survey remained online for three weeks in total (from April 29 to May 15, 2010).

The present study employs an individual project as the fundamental unit of analysis. Thus each respondent was asked to fill in the survey for only the last project he/she worked on. A function provided by the Qualtrics Research Suite prevented participants from completing the survey more than once.

To ensure the clarity of the survey, a pilot test was conducted among five practitioners. Their feedback contributed to the improvement of the layout of the questionnaire and its wording. The pilot testers were particularly asked to check any ambiguous or misleading questions which could lead to misinterpretation and therefore biased or invalid data. 


\subsubsection{Survey questions}

The survey questions and scales were developed prior the beginning of the interviews. Whenever possible, these questions were based on instruments used in previous studies, particularly Misra et al. (2009) and Strode (2005). This was important to ensure content validity, that is, that the survey questions measured correctly the constructs they were intended to measure. However, the study was primarily exploratory in that new measures had to be devised for 19 of the 34 questions.

The interviews' findings then helped define a parsimonious set of key concepts (Appendix F) so as to get a short but effective survey instrument. The findings were particularly valuable because input from project workers was gathered prior to the beginning of the survey on a larger scale. Thus in the survey there were one or two questions per construct as defined previously. Constructs were grouped in clusters as defined in the research model. The survey was short (taking only five to eight minutes to complete) and simple so as to increase the response rate by reducing the effort the participants had to make. All the constructs in the research model were measured on a Likert scale from 1 to 5 , or converted to such a scale. The final survey questions, selected and refined according to the interview findings, are presented in Appendix E.

There were five groups of questions in the survey corresponding to the four clusters described in the research model (in Figure 2-10 \& Figure 2-11, excluding SDM fit) plus demographic questions. The latter were questions about the experience of the respondent in software development, the organization and industry in which the project was conducted, and the position of the respondent in the project.

\subsubsection{Data analysis}

The goal of data analysis was to test the two parts of the research model and therefore highlight the relationships between the factors studied. The aim was to determine the most important factors in SDM fit and project success as described previously. 
Multivariate statistical methods were used to identify the contribution of each variable in the research model. To conduct this kind of analysis, the first step was the development of a conceptual model to express hypotheses about the relationships among variables (Easterby-Smith et al., 2008). The model presented in the previous chapter includes both observed variables, which are directly measured (for example, criticality), and latent variables, which are not measured directly but inferred from observed variables (for example, project success).

Shenhar et al. (2002) explain that multivariate methods have often been used to study the concept of fit in contingency theory. For the current research, a p-value (i.e. significance level) of .05 was used to evaluate the degree to which the model was supported.

In this study, data analysis included:

- Descriptive statistics

- Factor analysis

- Correlation analysis

- Linear multiple regression analysis.

\section{Descriptive statistics}

Descriptive statistics first describe, in a general way, the data gathered through the survey. It presents simple summaries of the variables measured with the mean, standard deviation, minimum and maximum of each item. In addition, it provides some background information on the sample through an analysis of the demographic questions.

\section{Factor analysis}

An exploratory factor analysis was then performed on measures of the project and project environment to evaluate if some of the variables measured the same underlying concept. This kind of analysis identifies multidimensional constructs and its purpose is to reduce the number of variables in the model. 
An exploratory factor analysis was also undertaken on the SDM and project success clusters. This enables the construct validity of these variables to be evaluated. If, as expected, the observed variables in each cluster measure the same underlying construct, then there should be only one factor identified by the factor analysis.

The technique used was a principal component analysis, which is appropriate for exploratory research.

\section{Correlation analysis and linear multiple regression analysis}

Correlation analysis looked at the associations between sets of variables. It resulted in two sets of values: (i) the Pearson's coefficient of correlation and (ii) the values of significance. In this study, the correlation tests were conducted at level $p<.05$. In other words, only hypotheses supported at $p<.05$ were accepted. Although the correlation coefficients inform us of the degree of relationship between the independent variables and the dependent variable, the correlation analysis does not the measure the causality. The causality may, however, be inferred by other tests such as multiple regression analysis.

A multiple regression analysis is appropriate for the current study as it explores the hypothesized causal relationships between multiple independent variables and a dependent variable (Wagner, 2010). Regression coefficients within a regression model or equation convey the relative contribution of independent variables to the estimation or prediction of a dependent variable of interest, say the extent of SDM fit or the likelihood of project success. The correlation coefficient $\mathrm{R}^{2}$ provides an aggregated measure of how well a regression model or equation makes such estimates, and is often referred to as a measure of fit between estimated and actual values.

As the research model is divided into two parts, the correlation and regression analyses were conducted twice, once for each part.

First, project environment and project were the independent variables and SDM was the dependent variable. These relationships are represented by $\mathrm{H} 1$ and $\mathrm{H} 2$ in the research model (Figure 2-10). As it aims at determining the important factors in 
SDM fit, the analysis was conducted on successful projects. It is indeed considered that when a project is successful, there is SDM fit (SDM fit being a necessary condition of project success). A correlation test on these projects therefore revealed the important factors in SDM fit.

Based on the preliminary correlation analysis, factors to be included in the multiple regression analysis were chosen. The regression analysis tested the following model/equation:

$$
y=a_{0}+\sum_{i=1}^{j} a_{i} x_{i}+\beta
$$

$y$ is the $S D M$ utilized on a scale from pure plan-driven to pure agile. $x_{i}$ are the dependent factors identified by the factor analysis. The number of factors that were included $(j)$ was determined by the preliminary correlation analysis. $a_{0}$ is a constant and $\beta$ is the residual, which should ideally follow a normal distribution. The analysis determined $a_{i}$ for each $x_{i}$, which informed us of the relative predictive importance of the associated factors in the choice of SDM.

The regression model was used to calculate, for each project, a score for the SDM predicted to be appropriate for the project circumstances. The absolute difference between that predicted score and the empirically observed score for the SDM-in-use, i.e., the residual term in the regression equation was then used as a proxy measure for what we define as "SDM fit", that is, as a measure of SDM fit. A large absolute value indicates poor fit and a small absolute value indicates a good fit ('a methodology that fits').

We may find projects that present a high fit measure but little success. This is because SDM fit is a necessary but not sufficient condition to obtain project success. These projects have failed for reasons unrelated to SDM fit.

In the second part of the analysis, project environment, SDM fit and project were the independent factors and project success was the dependent variable. These relationships are represented by $\mathrm{H} 3, \mathrm{H} 4$, and $\mathrm{H} 5$ in the second part of the research model (Figure 2-11). The role of all these variables in project success was first evaluated by a correlation analysis and then a linear multiple regression analysis. 
Insomuch as project environment, SDM fit and project precede project success, this analysis revealed whether these factors have a causal link with project success and to what extent these links explains the variability of project success.

The regression analysis determined the impact of the independent variables on Project Success, according to the following equation:

$$
z=a_{0}+\sum_{i=1}^{j} a_{i} x_{i}+\beta
$$

$z$ is Project Success (a one-dimensional factor) on a scale from very unsuccessful to very successful. $a_{0}$ is a constant and $\beta$ is the residual. The number of factors that were included $(j)$ was determined by the preliminary correlation analysis. $x_{i}$ are the independent factors, and $a_{i}$ are their associated coefficients. The goal is to evaluate these coefficients to determine the important factors that impact project success.

\subsubsection{Ethical considerations}

Human Ethics Committee approval, separate from that granted for the interviews, was obtained from Victoria University of Wellington for the survey. The survey was anonymous and the final results only presented in an aggregated form. Thus it is impossible to identify any company and project from the data collected or reported. Further, raw data were only accessible to the researcher and his supervisor and stored in a password-protected file, which was deleted at the end of the study. Targeted respondents were contacted by email to participate in the study. An information sheet was attached to the email (see Appendix D).

\subsubsection{Resources}

As the survey was conducted online, the cost was very low, and all the resources needed were already at the disposal of students at Victoria University of Wellington. The Qualtrics Research Suite was used to design and conduct the survey. The use of emails to contact the respondents did not add any cost to the project either. After the data gathering, SPSS (Statistical Package for the Social Sciences) also available on 
University computers was used to analyse the research data. Consequently, there was no extra cost associated with the use of this questionnaire instrument.

\subsection{Credibility of the Research and Generalizability}

The two aspects of credibility of the research - reliability and validity - are both discussed below, as well as the generalizability issue.

\subsubsection{Reliability}

A study is reliable if the same results are obtained when the study is repeated several times (Collis \& Hussey, 2003). For the survey, it can be difficult to get an unbiased sample. But by using a quota sampling, diverse opinions from different people representing the target population of project workers was collected. To measure reliability, a survey can be administered twice to the same person or to two different persons who worked on the same project. This option is not possible for the survey as it was anonymous. Another option to measure is to use several questions in the survey that measure the same construct. As this study is exploratory many variables were measured by a single question. However, reliability was tested for variables such as project success that were measured by several questions. In the current research, reliability is estimated by internal consistency, and measured by the Cronbach's $\alpha$ method. It evaluates the inter-item consistency reliability of a composite scale, which is the correlation of items within a construct. For exploratory studies, it is agreed that a coefficient alpha of 0.6 could be deemed acceptable (Straub, Boudreau \& Gefen, 2004). This test was performed to measure the reliability of the constructs measured by several items in the survey.

\subsubsection{Validity}

A study is valid if the findings accurately measure and represent the situation being studied (Collis \& Hussey, 2003). The responses to a questionnaire may be highly 
reliable but not valid if the questions do not measure what they intend to measure. Several types of validity can be distinguished:

- Construct validity, meaning that the constructs are real and reliable and that their measurement by the instrument accurately represents the reality (Straub et al., 2004). The construct validity was checked by an extensive literature review, preliminary interviews and a pilot test, which allow confidence in the constructs.

- Internal validity, meaning that the model explains correctly the causal relationships between the variables. To ensure this, the research model was designed based on the literature review and was then refined by interviews with project workers. Nevertheless, the reality is complex and there are many variables that may influence the independent variables. The current research aims to indentify some of the most important of them.

- External validity, related to the generalizability of the findings is discussed in the paragraph below.

\subsubsection{Generalizability}

A study is generalizable if the research results can be applied to the population from which the sample has been drawn (Collis \& Hussey, 2003). We should be careful in the generalization of the results of this study since the participants were not entirely randomly selected. The key constructs were carefully defined from the literature and checked by project workers. Each community was represented by an approximately equal number of participants. The statistical analysis of the findings, presented in the following chapter, informed us of the degree of confidence we can have in the model. However, while generalizability is critically important in descriptive and predictive research, it plays a less important role in exploratory research.

\subsection{Conclusion}

This chapter developed two research instruments to identify the critical factors in SDM fit. First, interviews of project workers were designed to identify key variables 
in SDM fit and to generate and refine a set of measurement items. Second, a survey of software development project workers was developed to provide statistical evidence to support the critical factors. The data analysis procedure was also presented in this chapter. The findings and data analysis is presented in the next chapter. 


\section{CHAPTER 4: DATA ANALYSIS AND RESULTS}

The fourth chapter presents the execution of the research instruments as presented in chapter three. It first describes the data gathered through interviews in order to refine the set of variables to be measured in the survey. Then the data collected from the questionnaires is presented and studied. Statistical analyses of survey data are then performed and presented. Lastly, the limitations of the tests conducted are acknowledged and a summary of the key findings is presented.

\subsection{Analysis of Interview Data}

\subsubsection{Profile of the interviewees}

In total, eight interviews were conducted in Wellington between mid-March and mid-April 2010. While the participants were primarily project managers, academics having an expertise and/or experience in project management and software development were also interviewed. The table below (Table 4-1) shows their classification by project management community of practice and economic sector (government, private, and education).

\begin{tabular}{ccccc}
\hline & PRINCE2 & PMI & Agile & Academics/Experts \\
\hline $\begin{array}{c}\text { Government } \\
\text { sector }\end{array}$ & 1 & 1 & 1 & \\
Private sector & 1 & 1 & 1 & 3 \\
\hline Education sector & & & & \\
\hline
\end{tabular}

Table 4-1: Interviewees' profile

Note: One of the interviewee had both PRINCE2 and PMI certifications and had worked on both PRINCE2 and PMI projects. He is counted in both corresponding columns in the table, which explains why the total of the table is 9.

All the practitioners who participated in the study had significant experience in project management and software development. Despite the fact that they belonged to different communities of practices, a pretty high level of agreement between interviewees was found. In the two next sections, the project environment and project 
variables listed in Table 3-1 and Table 3-2 respectively are ranked in order of their importance in influencing the choice of SDM. A summary of interviewee's verbal explanations is also reported. Interviewees will be named I1 to I8 to preserve their confidentiality.

\subsubsection{Project environment cluster}

\begin{tabular}{ll}
\hline Name of the variable & Number of points \\
\hline Methodology supported by top management & 30 \\
\hline Level of entrepreneurship & 27 \\
\hline Market uncertainty & 24 \\
\hline Economic sector & 18 \\
\hline Governance and compliance factors & 16 \\
\hline Power relations & 15 \\
\hline Project workers' experience with one & 12 \\
\hline methodology & 10 \\
\hline Project manager's experience with one & 10 \\
methodology & 9 \\
\hline Size of the organization & 7 \\
\hline Nature of the contract & 7 \\
\hline Project manager's certifications or training & \\
\hline Project workers' certifications or training & \\
\hline
\end{tabular}

Table 4-2: Ranked project environment variables

Table 3-1 listed (in alphabetical order) three groups of project environment variables: external environment variables, organizational variables, and people/community asset variables). In total, the three categories contained 12 project environment variables. Each variable was given voting points by interviewees according to its importance in determining the best-fit SDM and the points were added up to obtain the ranked list presented in Table 4-2. Project environment variables prompted considerably more comments from participants than did the next cluster (project variables). This suggests that, in spite of the focus on project factors in the literature, the environment is in practice often an important deciding factor in methodology selection. Each of the three categories of variables is briefly discussed in the following paragraphs for the purpose of generating a refined set of survey questions. 


\subsubsection{External environment variables}

\begin{tabular}{ll}
\hline Name of the variable & Number of points \\
\hline Market uncertainty & 24 \\
\hline Economic sector & 18 \\
Governance and compliance factors & 16 \\
\hline Power relations & 15 \\
\hline
\end{tabular}

Table 4-3: Ranked external environment variables

Market uncertainty was included in the survey. It is seen as an important contingency variable for interviewees because it informs the level of planning that can be used. The more uncertainty there is, the less appropriate plan-driven methodologies are, according to I2.

Economic sector was included in the survey. The economic sector makes a difference for many interviewees although several examples of agile projects within the government sector have been mentioned. The economic sector is actually believed to have an important impact on the attitude towards risk discussed previously. All interviewees in the government sector mentioned the high level of risk aversion in this sector, which therefore requires appropriate SDMs. The government sector is also associated with conservatism. It seems that the priority for the government is to get the product delivered whatever the duration and cost of the project (I5), whereas the private sector puts a high priority on time to market. The economic sector variable will therefore not be directly included in the survey as it is best represented by other project environment variables (like entrepreneurship and attitude towards risk). It was, however, tested in the survey (though inclusion as a demographic question) to evaluate if there is any major difference between the two groups.

Governance and compliance factors was not included in the survey. Interviewees think that governance and compliance variables can be important in project management but these do not necessarily influence the choice of plan-driven or agile methodologies. Furthermore, governance is a complex construct difficult to accurately measure in a survey. Most of the respondents linked governance to executive and top management buy-in. Further, I5 explains that guidelines can be 
interpreted and both development approaches can be tailored to fulfil compliance variables.

Power relations was included in the survey in the modified form of power distance. Interviewees saw power relations as influencing the empowerment of the project team and their ability to make important decisions. Not all of the interviewees had worked in diverse cultural environments. Those that had not were therefore unable to speak to the importance of this variable. I4, however, stated that agile methodologies are harder to implement in Asian cultures, because there is a high power distance and project team members are not empowered. He illustrated that concept by the following rhetorical questions: "Who am I allowed to talk to? Am I able to have a conversation with the CIO in the corridor?". The response to these questions is directly linked to the culture of the country in which the project is conducted. Hofstede's Power Distance Index was used to measure that concept (Hofstede \& Hofstede, 2005). It measures, on a scale from 1 to 120, the national culture and respect for authority. New Zealand has a low score of 22, whereas Malaysia has a high score of 104. These measures are in accordance with I4's description of doing work in both Malaysia and New Zealand.

As a result of the rankings and related discussion on the four variables in the external environment category, market uncertainty was included as an independent variable, economic sector was included as a demographic variable, and power relations was included as power distance. The remaining variable (governance and compliance factors) was excluded.

\subsubsection{Organizational variables}

\begin{tabular}{ll}
\hline Name of the variable & Number of points \\
\hline Methodology supported by top management & 30 \\
Level of entrepreneurship & 27 \\
Size of the organization & 10 \\
Nature of the contract & 9 \\
\hline
\end{tabular}

Table 4-4: Ranked organizational variables 
Methodology supported by top management is the variable that has received the largest number of points from interviewees and was included in the survey. They believe that without executive support and buy-in to the methodology used, it is very difficult to conduct the project. Executives have much control and power and can stop the project if needed. Project managers highlighted the need to generate the buyin of top management. Related to this variable, interviewees also often talked about organizational politics. Interviews stated that organizational politics has a much more important influence on how the project is conducted than the actual characteristics of the project. I5 explained that in the government sector, decisions may come directly from the minister who may impose the same approach as in previous projects that were successful, although the projects characteristics may be very different.

Level of entrepreneurship received the second largest number of points from interviewees and was included in the survey. Interviewees also emphasized the effect of the organizational culture and particularly the attitude towards risk. The latter is partly reflected by the level-of-entrepreneurship variable, but it was decided to add another measure (level of risk-taking willingness) to the survey to get a more accurate description of this construct. This variable evaluates whether the organization is risk averse, or whether it encourages risk-taking behaviour. Organizations that encourage risk-taking behaviour will more easily accept more "adventurous" methodologies (I2). On the other hand, I3 explains that when the organization is risk-averse, nobody wants to change the established norms and the way software is developed.

One may think that entrepreneurship is strongly correlated to the size of the organization but I1 mentions examples of big organizations in which there are processes in place to support innovative behaviour. Nevertheless, some interviewees found that large organizations have difficulties in adopting agile methodologies as they already have so many processes in place. However, I2 knows organizations where both agile and waterfall projects are running at the same time. As a consequence size of the organization was not included in the survey as a contingency (independent) variable. However it will be included as a demographic variable and is therefore available for analysis.

The nature of the contract has little importance for interviewees. Some of them have worked on agile projects with fixed price contracts. In that case, the scope becomes 
the adjusting variable in the iron triangle (cost, scope and time). As a consequence nature of the contract was not included in the survey.

As a result of the rankings and related discussion on the four variables in the organizational category, two existing variables (methodology supported by top management and level of entrepreneurship) were included in the survey along with a new variable (level of risk-taking willingness). The remaining two variables (size of the organization and nature of the contract) were excluded.

\subsubsection{People /community asset variables}

\begin{tabular}{ll}
\hline Name of the variable & Number of points \\
\hline $\begin{array}{l}\text { Project workers' experience with one } \\
\text { methodology }\end{array}$ & 12 \\
$\begin{array}{l}\text { Project manager's experience with one } \\
\text { methodology }\end{array}$ & 10 \\
Project manager's certifications or training & 7 \\
\hline Project workers' certifications or training & 7 \\
\hline
\end{tabular}

Table 4-5: Ranked people/community asset variables

Unanimously, practitioners rejected the four variables related to project manager's and workers' experience and training with one methodology as important contingency variables. They explained that the project manager and workers are selected by the top management to match the requirements of the project. Project managers further explained that they do not have the power to make decisions about the SDM. Moreover, several of them emphasized that they have been assigned to a project that had already started and therefore all the decisions about the methodology had already been made. I6 also claimed that organizations do not listen to their project workers; their opinions on which methodology to select is usually not considered. Additionally, project managers and workers are replaceable and their training and experience will only influence the project they will be assigned to but not the SDM that should be used. If it is not possible to find the appropriate project manager or workers that match the methodology, then it is still possible to train them, even though it may take some time at the beginning of the project. 
Nevertheless, I2 stated that there was a limited supply of agile project professionals in Wellington, which may prevent some organizations from selecting that kind of methodology. This may explain why some interviewees gave a small number of voting points to these four variables as they may have a limited influence. I8 also mentions the existence of projects in small organizations where the project manager gets full responsibility and has the power to make the choice of a methodology.

The influence of the project management methodology (Figure 2-1) on the software development approach was not included in the cards because it is a broad construct encompassing many factors. For this reason it is not included in Table 2-4. However it was mentioned by several interviewees. Some of them believed that even though PRINCE2 or PMI, for example, claim to be usable with any system development life cycle, it is not always a good idea to combine PRINCE2 and an agile life cycle because of value conflict. For example I3, who manages projects via the traditional project management methodology, explained that he is not comfortable with agile because if requirements and specifications are always changing and he cannot keep up with reports and documentation.

In these discussions, interviewees were employing the concept of project management methodology as a shorthand notation or proxy for the types of attitudes, skills and knowledge (that collectively received a significant number of votes) that they associated with particular SDMs. It is likely that survey respondents will do likewise. This creates a dilemma for the researcher - should a popular concept (project management methodology) be included in the study when it is a broad term that both links and conflates project environment and project factors (Figure 2-1) and may be employed by survey respondents as a proxy for the dependent variable, SDM?

The dilemma was resolved by including in the survey project management methodology as a project environment factor, but analysing it separately from other variables. In addition, a somewhat related concept, experience of the respondent (in software development generally) was included as a demographic variable. 


\subsubsection{Project cluster}

\begin{tabular}{|ll}
\hline Name of the variable & Number of points \\
\hline Procedural empowerment & 26 \\
\hline Project uncertainty & 25 \\
\hline Project cost & 23 \\
\hline Customer commitment & 22 \\
\hline Co-location of the project team members & 19 \\
\hline Technological uncertainty & 18 \\
\hline Customer adaptability & 18 \\
\hline Experience level of the team & 18 \\
\hline Project criticality & 16 \\
\hline Project size & 15 \\
\hline Project duration & 13 \\
\hline Impact of lack of timely delivery & 13 \\
\hline Education level of the team members & 11 \\
\hline Team size & 9 \\
\hline Proportion of the organization affected & 7 \\
\hline Impact of failure to deliver the product & 7 \\
\hline
\end{tabular}

\section{Table 4-6: Ranked project variables}

The 16 project variables in Table 3-2 are grouped into five categories (criticality, personnel, dynamism, culture of the project team, and people management). Each variable was given voting points by interviewees according to its importance in determining the best-fit SDM and the points were added up to obtain the ranked list presented in Table 4-6. Each category of project variables is briefly discussed in the following paragraphs for the purpose of generating a refined set of survey questions.

\subsubsection{Criticality}

\begin{tabular}{ll}
\hline Name of the variable & Number of points \\
\hline Project criticality & 16 \\
Impact of lack of timely delivery & 13 \\
Impact of failure to deliver the product & 7 \\
\hline
\end{tabular}

Table 4-7: Ranked criticality variables 
Project criticality was included in the survey. In this cluster, the project criticality variable appears slightly more important than impact of lack of timely delivery which was excluded along with impact of failure to deliver the product. However, no single variable from this category was selected as having the highest importance by interviewees. They all considered that these variables can each inform the methodology but are not key deciders. Respondent I2 explains that project criticality mainly informs the level of risk management needed rather than the choice of a methodology. In addition, I1 disagrees with the literature and argues that agile methodologies are better for highly critical projects as they help reduce defects in the final product. In summary, interviewees felt that the second and third variables did not significantly determine choice of SDM.

\subsubsection{Personnel}

\begin{tabular}{ll}
\hline Name of the variable & Number of points \\
\hline Experience level of the team & 18 \\
Education level of the team members & 11 \\
\hline
\end{tabular}

Table 4-8: Ranked personnel variables

Experience level of the team was included in the survey, and education level of the team members was excluded. Referred to as team maturity by certain interviewees, experience level of the team is seen as much more important than education level. Team members can be trained relatively quickly but developing experience takes longer. I2 explains that if a team lacks experience, it is more appropriate to choose a methodology with more structure and more pre-identified processes (i.e. a traditional methodology) to correctly guide the project team members.

\subsubsection{Dynamism}

\begin{tabular}{ll}
\hline Name of the variable & Number of points \\
\hline Project uncertainty & 25 \\
Technological uncertainty & 18 \\
\hline
\end{tabular}

Table 4-9: Ranked dynamism variables 
Project uncertainty and technological uncertainty were both included in the survey. Interviewees agreed that the more uncertainty there is in a project the more appropriate agile methodologies are because their iterative approaches allow change during the development process. On the other hand, I1 explains that if the project uncertainty is very low, then a linear approach is sufficient. I4 warns us, however, that it is not always appropriate to respond to change. In particular, over-responding to change is very expensive and the product may not always need to respond to all the changes.

Similarly, technological uncertainty has an impact on methodology selection. I2 explains that if the technology is well known, there will be fewer unexpected difficulties and conformance to plan will be easier.

\subsubsection{Culture of the project team}

\begin{tabular}{ll}
\hline Name of the variable & Number of points \\
\hline Procedural empowerment & 26 \\
Customer commitment & 22 \\
Customer adaptability & 18 \\
\hline
\end{tabular}

Table 4-10: Ranked culture variables

Procedural empowerment was included in the survey. Participants agreed that the culture of the project team, and procedural empowerment in particular, are important elements in SDM fit. Interviewees explained that the culture must be aligned with procedures that enable the project team to work in a way that suits them. For the project team to respond rapidly to change (either in actual requirements or perceived requirements) they need to be granted the power of decision making over how to proceed (procedural empowerment).

Customer commitment was included in the survey. The customer who is part of the project team in agile projects is seen as very important in the choice of a SDM. I1 recalls an agile project that he declined because the project owner did not want to make somebody available at least half-time to the project team. Without customer commitment and support, agile projects are jeopardized. 
Interviewees also think that the customer adaptability in selecting various approaches is important, though I3 claims that customers are often not adaptable at all. I8 explains that, in certain situations, the way the software is developed is not fully disclosed to the project owner, which leaves some margin for the project team to tailor their SDM. Thus, customer support and commitment are perceived as more important than customer adaptability. Accordingly customer adaptability was replaced in the survey by a new variable customer support.

\subsubsection{People management}

\begin{tabular}{ll}
\hline Name of the variable & Number of points \\
\hline Project cost & 23 \\
\hline Co-location of the project team members & 19 \\
\hline Project size & 15 \\
\hline Project duration & 13 \\
\hline Team size & 9 \\
\hline Proportion of the organization affected & 7 \\
\hline
\end{tabular}

Table 4-11: Ranked people management variables

Project cost (measured in \$) was included in the survey as an integral component of project size (measured in man years). Certain interviewees see the project cost as unimportant in terms of SDM selection. Their argument is that the investment can be split in parts of a reasonable size so that agile methodologies may be used on large projects. On the other hand, other interviewees rate this variable as highly important since it determines the level of processes and control in place for the whole project, and thus has an important influence on the SDM. Project cost is the variable that represents best the size of the project according to most interviewees. I3 explains that, in his organization, if the budget is less than NZD 50,000 then agile methodologies can be used, otherwise too much coordination and control is required when mounted in a project environment not already amenable to agile methodologies.

Co-location of the project team members was included in the survey. Co-location is a requirement for agile methodologies, but some agile projects have been successfully managed with teams located in different places (I1). Nevertheless, co-location is still 
an important deciding variable for the interviewees. I5 emphasizes the importance of this variable in terms of relationship building and communication, which is paramount to agile methodologies.

Project duration and team size (measured in number of team members) were excluded from the survey. Interviewees concurred that both concepts can be subsumed under project cost and project size, and that additional measures were unnecessary.

Finally, proportion of the organization affected was excluded from the survey. This concept informs us on the diversity of stakeholders (I6). However according to the interviewees, this does not have much influence on the choice of a methodology.

In summary, nine variables from the project cluster were included in the survey (one of which has two components). These are named P1-P9 in Table 4-12.

\subsubsection{Conclusion}

To conclude, the main finding from these interviews is that organizations often do not think objectively about methodology selection; rather this decision is imposed from top management or the same methodology is used on every project. Power relations or organizational politics are, interestingly, considered by most interviewees to have an important influence in practice although they might be problematic in their influence on the selection of the SDM that fits other aspects of the project environment and project. Hence, most participants discussed this aspect. The past habits variable (i.e. the way software is generally developed in a particular organization) has also been brought up by several participants to explain the way organizations usually conduct their projects, without being conscious of the need to undertake a SDM selection process.

A few interviewees explained how they recognize if and when the SDM is appropriate for a given project. I4 explains that when the wrong approach is adopted, there are non-stop problems, which prevent the project from being completed on time and within budget. I5 mentioned that when the project team does not like the methodology, they may spend more time fighting the methodology than doing actual 
work. This confirms the importance of including team members' attitude towards the methodology in measuring success.

All in all, interviews helped to reduce the number of relevant variables from 28 (in Table 3-1 \& Table 3-2) to 16 . The most important variables in each category have been selected on the basis of both the number of points allocated (quantitative) and interviewee's comments (qualitative). The variables selected to be measured in the survey are listed in the following table (Table 4-12).

In appendix F, an operational definition is provided for each of these variables as well as their polarity.

\begin{tabular}{|c|c|c|c|}
\hline \multicolumn{2}{|c|}{ Project Environment Variables } & \multicolumn{2}{|c|}{ Project Variables } \\
\hline $\begin{array}{l}\text { PE1 } \\
\text { man }\end{array}$ & $\begin{array}{l}\text { Methodology supported by top } \\
\text { ment }\end{array}$ & P1 & Project criticality \\
\hline PE2 & Level of entrepreneurship & $\mathbf{P 2}$ & Experience level of the team \\
\hline PE3 & Level of risk-taking willingness & P3 & Technological uncertainty \\
\hline PE4 & Market uncertainty & P4 & Project uncertainty \\
\hline PE5 & Power distance & P5 & Procedural empowerment \\
\hline PE6 & Project Management Methodology & P6 & Customer commitment \\
\hline \multirow[t]{3}{*}{ PE7 } & Economic sector & P7 & Customer support \\
\hline & & P8 & Project Size \\
\hline & & P9 & $\begin{array}{l}\text { Co-location of the project team } \\
\text { members }\end{array}$ \\
\hline
\end{tabular}

Table 4-12: Dependent variables to be measured in the survey

\subsection{Analysis of Survey Data}

\subsubsection{Refinement of the survey}

As a result of the interviews, the survey was refined via a three-step process. First, operational definitions were created for the 16 variables identified in Table 4-12. These are enshrined in the survey questions (Appendix E) and variables (Appendix F), which describe their polarity, that is, the direction of their expected impact on choice of SDM on a scale from pure plan to pure agile. Second, the web-based survey instrument was developed for the Qualtrics site and feedback received from 
people with experience in survey design and development, or experience in the field of software development, or both. Third, a pilot was conducted among project management practitioners. Their feedback resulted in the rewording of certain questions to avoid ambiguity or bias in the response. The final set of questions, variables, associated codes and polarities are presented in Appendix E. The survey contains 16 independent variables that are clustered to form the two groups in the research model: project environment and project. Each independent variable, except project size, was measured by a single question and each represented a separate construct or dimension of a construct.

\subsubsection{Preparation of the data}

Before the data analysis started, data needed to be prepared. First, certain variables needed to be coded on a scale from 1 to 5 . This is the case for project cost, project size and country (which will be translated into the Power Distance for that country). For the project cost, the amount was first converted into US dollars and then to the categorization below (Table 4-13). Similarly, the project size was converted to man months and then to a scale from 1 to 5 (Table 4-13). For each country, Hofstede's Power distance Index (PDI) was used. It gives a score on a scale from 1 to 120 , which was then converted to a scale from 1 to 5 .

\begin{tabular}{lll}
\hline Code & Project cost in USD & Project size in man hours \\
\hline $\mathbf{1}$ & Less than 100,000 & 1 to 10 \\
$\mathbf{2}$ & 100,000 to $1 \mathrm{M}$ & 11 to 50 \\
$\mathbf{3}$ & $1 \mathrm{M}$ to $10 \mathrm{M}$ & 51 to 100 \\
$\mathbf{4}$ & $10 \mathrm{M}$ to $100 \mathrm{M}$ & 101 to 500 \\
$\mathbf{5}$ & More than $100 \mathrm{M}$ & $501+$ \\
\hline
\end{tabular}

Table 4-13: Codes used for the project cost and project size variables

The project management methodology was also coded on a scale from 1 to 5. Plandriven project management methodologies like PRINCE2 were coded 1. PMI (PMBOK) as a broad middle-of-the-road methodology was coded 3. Agile project management methodologies were coded 5. Other approaches, such as 'in-house project management methodologies' were included in the analysis only if there was 
sufficient indication on their position along the plan-to-agile spectrum. Finally, the economic sector was coded on a two-point scale (1- Private Sector, 2- Public sector).

As described in the following paragraphs, a new variable was created by factor analysis for SDM and project success. For these variables, no missing values were found because of a feature in Qualtrics that forces the participant to respond to the questions. While, participants had the opportunity to respond "Don't Know" or "Not Applicable", all respondents answered each SDM item, and nearly all respondents answered each project success item.

Finally, three scales needed to be reversed so that all the independent variables had the same polarity (i.e. the same linear relationship with the Software Development Methodology variable): power distance, criticality, and project size. Thus all the independent project environment and project variables, and SDM, were measured on a scale from 1- Pure plan-driven to 5- Pure agile. Project Success was measured on a scale from 1-Unsuccessful to 5-Successful.

21 responses were excluded from the database before analysis. Two of them were dismissed because the methodology changed during the course of the project. The rest were incomplete responses (for example, participants who responded 'don't know' to the key questions), which prevented these projects from being included in the analysis. In total, 106 responses of the 127 surveys received were selected for initial data analysis.

It is difficult to estimate the response rate as participants were asked to forward the survey to colleagues; these forwards were untracked. In addition, links to the survey were published by certain participants on blogs or websites. The total number of emails that were sent to invite participation in the survey is 452. Nearly all participants completed the survey within four to seven minutes.

\subsubsection{Descriptive statistics}

Data collected in the first part of the survey give background information on the participants and the projects being analysed. They are presented in Table 4-14. 
This table shows that there is a good variety of projects included in the database. The majority of the respondents $(66 \%)$ have more than 10 years of experience in software development (DEM1). For future research, the scale could be adapted to better represent the distribution of years of experience.

There was a large variation in organization size (DEM2). The most represented category is that of organizations which have more than 5,000 employees. This can be explained by the fact that a majority of the participants were from the United States of America (Table 4-15), where there are some very big organizations. For future research, the scale could be adapted to better represent the distribution of the organizations' size.

Projects were conducted in a large number of different industries (DEM3). The Finance/Insurance industry is the one most represented followed by the IT services industry and the government sector. Economic sector was derived from industry by separating out government (coded 1) from all other industries, all of which are from the non-government or private sector (coded 5). By this measure most projects occurred in the private sector.

Participants had a variety of positions in the project (DEM4). Of the respondents, $43 \%$ were project managers (either agile or traditional).

The variable from the Project section (P8.1: Cost) revealed that the smallest project cost USD 2,500 (2 man months) and the biggest cost USD 790 million (1,260 man years). As suggested by one of the pilot testers, not all respondents knew the total cost of the project they worked on. This question was answered by only $54 \%$ of the respondents. This shows that all the people involved in a project do not always know all the characteristics of the project. $70 \%$ of the respondents that did not answer the question were team members or team leaders. $28 \%$ were project managers and perhaps some of these did not want to disclose the cost of the project. Another reason might be that there is not always a clear separation between projects that are interconnected. It is therefore more difficult in these cases to evaluate the cost of a single project.

The variable from the Project Environment section (PE6: Project management methodology) revealed that a little more than half $(52 \%)$ of respondents identified 
that the project management methodology (PE6) was Agile Project Management. PRINCE2 accounted for 9.5\%, and 15\% PMI (PMBOK) 15\%. This suggests that the agile community is over-represented in the sample, which may not be inappropriate for an exploratory study conducted at a time when considerable research is focussed on Agile. In addition, while PMI (PMBOK) and PRINCE2 are more specific project management methodologies, Agile Project Management is broader and may vary a lot from one project to another. It is likely that more than a few of these variations are included in the 55 projects managed via Agile. The commonality in the agile projects is that they are all based on the agile philosophy, which provides a measure of the degree of alignment between the project management and SDMs (Figure 2-1).

\begin{tabular}{|c|c|c|c|}
\hline $\begin{array}{l}\text { DEM1: Experience of the } \\
\text { respondent }\end{array}$ & $N=106$ & $\begin{array}{l}\text { DEM2: Organization size (Number } \\
\text { of employees) }\end{array}$ & $N=106$ \\
\hline Less than 1 year & 0 & 1 to 10 & 7 \\
\hline 1 to 2 years & 4 & 11 to 50 & 19 \\
\hline 2 to 5 years & 9 & 51 to 100 & 10 \\
\hline 5 to 10 years & 23 & 101 to 500 & 21 \\
\hline more than 10 years & 70 & 501 to 1,000 & 5 \\
\hline \multirow{17}{*}{$\begin{array}{l}\text { DEM3: Industry } \\
\text { Finance/Insurance } \\
\text { Manufacturing } \\
\text { Education } \\
\text { Pharmaceutical/Healthcare } \\
\text { Computer related } \\
\text { Construction/Utilities/Engineering } \\
\text { Marketing/Retail } \\
\text { Government } \\
\text { IT Services/Vendors } \\
\text { Transportation } \\
\text { Real Estate/Legal Services } \\
\text { Aerospace } \\
\text { Media/Publishing } \\
\text { Other (includes Mining, Research, } \\
\text { Telecommunication, Energy \& Non- } \\
\text { profit) }\end{array}$} & \multirow{2}{*}{$\begin{array}{l}N=106 \\
18\end{array}$} & 1,001 to 5,000 & 19 \\
\hline & & More than 5,000 & 25 \\
\hline & 9 & DEM4: Position of the respondent & $N=106$ \\
\hline & \multirow{3}{*}{$\begin{array}{l}7 \\
6 \\
7\end{array}$} & Traditional Project Manager & 29 \\
\hline & & Agile Project Manager & 17 \\
\hline & & Traditional Team Leader & 12 \\
\hline & \multirow{3}{*}{$\begin{array}{l}4 \\
4 \\
11\end{array}$} & Agile Team Leader & 9 \\
\hline & & Team member: Developer/tester & 21 \\
\hline & & Architect & 4 \\
\hline & 13 & Other (includes delivery, & 14 \\
\hline & \multirow{2}{*}{$\begin{array}{l}3 \\
7\end{array}$} & development or testing manager & \\
\hline & & & \\
\hline & \multirow{2}{*}{$\begin{array}{l}4 \\
5\end{array}$} & PE6: Project Management & $N=106$ \\
\hline & & Methodology & \\
\hline & \multirow[t]{3}{*}{8} & PRINCE2 & 10 \\
\hline & & PMI (PMBOK) & 15 \\
\hline & & Agile Project Management & 56 \\
\hline P8.1: Project cost (USD) & $N=57$ & None & 8 \\
\hline Less than 100,000 & 8 & Don't Know & 8 \\
\hline 100,000 to $1 \mathrm{M}$ & 18 & Other (includes mainly in-house & 9 \\
\hline $1 \mathrm{M}$ to $10 \mathrm{M}$ & 21 & methodology) & \\
\hline $10 \mathrm{M}$ to $100 \mathrm{M}$ & 7 & & \\
\hline More than $100 \mathrm{M}$ & 3 & & \\
\hline
\end{tabular}

Table 4-14: Characteristics of the survey sample 
In section 4.1.2.3, it was suggested that participants' responses to questions about software development methodology (SDM) and project management methodology (PE6) are likely to be conflated, and that project management methodology should be analyzed separately. The relevant descriptive statistics will be briefly investigated at this point. Of the 21 projects based on a linear/waterfall SDM, eight (38\%) were associated with PRINCE2, six (29\%) were associated with PMI (PMBOK), and none $(0 \%)$ were associated with an agile project management methodology. Of the 24 projects based on an iterative and incremental SDM, one (4\%) was associated with PRINCE2, six (25\%) were associated with PMI (PMBOK), and six (25\%) were associated with an agile project management methodology. Of the 61 projects based on an agile (iterative, incremental and adaptive) SDM, none (0\%) were associated with PRINCE2, two (3\%) were associated with PMI (PMBOK), and 50 (82\%) were associated with an agile project management methodology.

While 106 responses were obtained to Question 14 (SDM2), only 81 responses to Question 7 (PE6: Project management methodology) could be mapped onto a continuum from PRINCE2 to agile. The remaining 25 responses indicated an inhouse project management methodology or an informal or unknown methodology. Of the 81 responses, 10 (12\%) projects used PRINCE2, 15 (19\%) used PMI (PMBOK), and $56(69 \%)$ used agile. This suggests that, as outlined in sections 2.1.2, 2.1.3, and 4.1.2.3, alignment between SDM and project management methodology is the norm. Lack of alignment may compromise project success. The fact that no linear/waterfall SDMs were associated with an agile project management methodology, and no agile SDMs were associated with PRINCE2, suggests respondents are describing projects in which SDM and project management methodology are aligned, or, as discussed in 4.1.2.3, conflated. The descriptive statistics support the intent to separate out project management method (PE6) from both project environment and SDM variables and the relationship between them.

Participants were from 22 different countries. The most represented country was the USA (36\%), followed by New Zealand (18\%) and Australia (15\%). Unfortunately all of these countries have a low PDI. The lack of responses from countries with a high PDI suggests that sampling restrictions prevent this variable from contributing to the variance. That is, this particular sample cannot test hypotheses regarding PDI. 
Data Analysis and Results

\begin{tabular}{lll|lll}
\hline Country & Frequency & Percentage & Country & Frequency & Percentage \\
\hline Argentina & 1 & .9 & Netherlands & 1 & .9 \\
\hline Australia & 16 & 15.1 & New Zealand & 19 & 17.9 \\
\hline Canada & 4 & 3.8 & Singapore & 1 & .9 \\
\hline Chile & 1 & .9 & South Africa & 2 & 1.9 \\
\hline Denmark & 1 & .9 & Spain & 1 & .9 \\
\hline France & 5 & 4.7 & Sweden & 1 & .9 \\
\hline Germany & 2 & 1.9 & United Kingdom & 4 & 3.8 \\
\hline India & 3 & 2.8 & United States of & 38 & 35.8 \\
\hline Iran & 1 & .9 & America & .9 \\
\hline Luxembourg & 1 & .9 & Viet Nam & 1 & .9 \\
\hline Malaysia & 1 & .9 & .9 & Temen & 1 \\
\hline Mexico & 1 & & & 106 \\
\hline
\end{tabular}

Table 4-15: Participants by country

Descriptive statistics were then performed on the main variables to describe the data collected by the survey. The mean, standard deviation, minimum and maximum of each variable are represented in the table below. In this table, all the variables are represented on a scale from 1- very low to 5- very high. Variables marked with ${ }^{*}$ will be reversed before data analysis (correlation and regression analyses) so that all the variables have the same polarity with the SDM (Table 4-16).

All the variables were measured on a scale from 1 to 5 ; the middle value is therefore 3. Most of these variables have a mean value close to 3 , a minimum of 1 and maximum of 5. All but one of the variables (PE5: power distance) has a standard deviation greater than 1 , suggesting a moderate degree of variability in the data.

Descriptive statistics for project environment and project variables are reported in Table 4-16. The very high mean for economic sector (PE7) reflects a sample in which 11 respondents were in the public sector and 95 were from the private sector. Respondents, who were disproportionately from the agile community, on average agreed that that the project management methodology was agile (PE6). Most respondents agreed that the experience level of project team members was high (P2), but are on the whole not very empowered (P5). Most respondents also agreed that project uncertainty (P4) was high (i.e. requirements were not very stable), that 
customer commitment (P6) was high, and that technological uncertainty (P3) was low (i.e. technology was well known by the project team).

Respondents were predominantly from USA, New Zealand and Australia, and the average power distance calculated for the sample is therefore low (PE5). Their responses indicate a low level of risk-taking willingness (PE3) and top management support for a more traditional and plan-driven approach (PE1).

\begin{tabular}{llllll}
\hline $\begin{array}{l}\text { Variable (From 1- very low to 5- very high, except } \\
\text { otherwise stated) }\end{array}$ & N & Mean & $\begin{array}{c}\text { Std } \\
\text { Dev }\end{array}$ & Minimum & Maximum \\
\hline $\begin{array}{l}\text { PROJECT ENVIRONMENT } \\
\text { PE1: Methodology supported by top }\end{array}$ & 104 & 2.62 & 1.33 & 1 & 5 \\
management (from 1-plan-driven to 5- agile) & & & & & \\
\hline PE2: Level of entrepreneurship & 106 & 3.27 & 1.26 & 1 & 5 \\
\hline PE3: Level of risk-taking willingness & 105 & 2.54 & 1.14 & 1 & 5 \\
\hline PE4: Market uncertainty & 106 & 2.68 & 1.31 & 1 & 5 \\
\hline PE5: Power distance* & 106 & 2.34 & 0.52 & 1.57 & 4.46 \\
\hline PROJECT & & & & & 5 \\
\hline P1: Project criticality* & 106 & 2.75 & 1.07 & 1 & 5 \\
\hline P2: Experience level of the team & 106 & 3.62 & 1.28 & 1 & 5 \\
\hline P3: Technological uncertainty & 106 & 2.28 & 1.14 & 1 & 5 \\
\hline P4: Project uncertainty & 106 & 3.45 & 1.24 & 1 & 5 \\
\hline P5: Procedural empowerment & 106 & 2.48 & 1.03 & 1 & 5 \\
\hline P6: Customer commitment & 106 & 3.45 & 1.20 & 1 & 5 \\
\hline P7: Methodology supported by the customer & 103 & 2.89 & 1.15 & 1 & 5 \\
\hline from 1-plan-driven to 5- agile) & 100 & 2.66 & 1.04 & 1 & 5 \\
\hline P8: Project size* & 106 & 3.33 & 1.46 & 1 & 5 \\
\hline P9: Co-location of the project team members & & & & 5 \\
\hline
\end{tabular}

* These scales will be reversed for data analysis

Table 4-16: Summarized descriptive statistics for the independent variables

Descriptive statistics for SDM and project success variables are reported in Table 4-17. The four software development variables have very similar statistics. Their means, which are greater than 3 , shows that on average an agile philosophy was more often adopted.

On average, projects were fairly successful. Time and budget score lower means, which indicate that, relative to other measures of project success, a few more projects 
tend to be completed after the deadline or at a higher cost than expected. The completion-on-budget question was answered 'Not Applicable' for seven projects. The latter were all agile projects, perhaps because there is not always a preestablished budget for this kind of project.

Finally, the project success variables associated with the more direct impact on the project team also have a slightly lower mean than the other measures of project success. The lower mean shows that project teams are not always satisfied with the way they worked, although they have delivered a good product. One of the survey respondents explained that stress can be one of the elements that reduce team satisfaction.

\begin{tabular}{lccccc}
\hline \multicolumn{1}{c}{ Variable } & N & Mean & $\begin{array}{c}\text { Standard } \\
\text { Deviation }\end{array}$ & Minimum & Maximum \\
\hline SOFTWARE DEVELOPMENT METHODOLOGY (1- plan-driven, & 5- agile) & & \\
\hline SDM1.1: Individuals over Processes & 106 & 3.31 & 1.13 & 1 & 5 \\
\hline $\begin{array}{l}\text { SDM1.2: Working code over } \\
\text { documentation }\end{array}$ & 106 & 3.58 & 1.19 & 1 & 5 \\
\hline SDM1.3: Collaboration over contract & 106 & 3.42 & 1.26 & 1 & 5 \\
\hline SDM1.4: Change over plan & 106 & 3.40 & 1.28 & 1 & 5 \\
\hline SDM2: Development life cycle & 106 & 3.75 & 1.60 & 1 & 5 \\
\hline PROJECT SUCCESS (1- unsuccessful, 5- successful) & & & & \\
\hline PS1.1: Time & 105 & 3.36 & 1.20 & 1 & 5 \\
\hline PS1.2: Budget & 99 & 3.34 & 1.22 & 1 & 5 \\
\hline PS1.3: Functionality & 105 & 4.13 & 1.02 & 1 & 5 \\
\hline PS1.4: Quality & 106 & 4.26 & 0.89 & 1 & 5 \\
\hline PS2.1: Addresses a need & 105 & 4.37 & 0.71 & 2 & 5 \\
\hline PS2.2: Product is used & 103 & 4.50 & 0.74 & 1 & 5 \\
\hline PS2.3: Customer is satisfied & 103 & 4.17 & 0.87 & 2 & 5 \\
\hline PS3.1: Team is satisfied & 106 & 3.73 & 1.06 & 1 & 5 \\
\hline PS3.2: Team would work the same & 102 & 3.75 & 1.24 & 1 & 5 \\
way again & & & & & \\
\hline
\end{tabular}

Table 4-17: Summarized descriptive statistics for the dependent variables 


\subsubsection{Test of reliability}

For testing the internal consistency of the project success, SDM, and project size variables, Cronbach's alpha test was employed. The test gave a value well above .8 for all the variables, which indicates that there is no problem with the internal consistency of these variables. Their constituent items can be considered as measuring the same factor. Exploratory, descriptive, and predictive research may be supported by these findings (Table 4-18).

\begin{tabular}{lll}
\hline Variable & Number of items & Cronbach's alpha \\
\hline Project Size (P8.1 and P8.2) & 2 & .873 \\
SDM (SDM1.1 to SDM2) & 5 & .877 \\
Project Success (PS1.1 to PS3.2) & 9 & .906 \\
\hline
\end{tabular}

Table 4-18: Reliability analysis

\subsubsection{Factor analysis}

The factors identified by the following analyses were used for the rest of data analysis. From this point onwards scales have been reversed for items PE5, P1, and P8. All the factors were normalized (i.e. they have a mean of 0 and standard deviation of 1). When a normalized independent factor has a value below 0 , it indicates the choice of a more plan-driven approach, while a value greater than 0 indicates the choice of a more agile approach. Project success is greater than 0 for successful projects and below the threshold of 0 for unsuccessful projects. In other words, the mean is used to distinguish successful from unsuccessful projects.

\subsubsection{Exploratory factor analysis of the project environment variables}

For the reasons discussed in section 4.2.3 (descriptive statistics) Project Management Methodology (PE6) and Economic Sector (PE7) and were excluded from this analysis of the project environment variables. Thus only PE1 to PE5 were included in the factor analysis. Three factors were identified. These explain $76.4 \%$ of the variability in the five Project Environment variables. 
The three factors obtained (PE_FAC1 to PE_FAC3) needed to be renamed to represent the construct they measure. PE_FAC1, which includes the attitude towards risk, the level of entrepreneurship and the methodology supported by executives, was named organizational culture (on a scale from conservative culture to entrepreneurial culture). The two other factors are mainly loaded by one variable each. Thus PE_FAC2 was named market uncertainty and PE_FAC3 low power distance. The scale of the power distance variable was reversed before this analysis because it was hypothesized that a low power distance would influence the choice of an agile approach. Consequently, the resulting factor PE_FAC3 represents power distance on a scale from high power distance to low power distance (Table 4-19).

\begin{tabular}{|c|c|c|c|}
\hline $\mathrm{X}$ & $\begin{array}{c}\text { PE_FAC1 } \\
\text { Organizational } \\
\text { culture (from } \\
\text { conservative to } \\
\text { entrepreneurial) }\end{array}$ & $\begin{array}{c}\text { PE_FAC2 } \\
\text { Market } \\
\text { uncertainty }\end{array}$ & $\begin{array}{c}\text { PE_FAC3 } \\
\text { Low power } \\
\text { distance }\end{array}$ \\
\hline $\begin{array}{l}\text { PE1: Methodology supported by top } \\
\text { management (from plan-driven to agile) }\end{array}$ & .748 & .246 & .050 \\
\hline PE2: Level of entrepreneurship & .746 & -.175 & -.096 \\
\hline PE3: Level of risk-taking willingness & .797 & .008 & .223 \\
\hline PE4: Market uncertainty & -.030 & .948 & -.230 \\
\hline PE5:Power distance* & -.158 & .197 & .949 \\
\hline
\end{tabular}

* This scale was reversed for data analysis.

Table 4-19: Factor analysis of the project environment variables

\subsubsection{Exploratory factor analysis of the project variables}

This analysis reduced the nine project variables to three factors. These three factors explain $56.6 \%$ of the variability in the nine project variables.

The first factor emphasizes empowerment of the team and methodology supported by the customer, which both load above .75 . These two variables arguably represent two dimensions of the project team empowerment: i) empowerment by executives; and ii) empowerment by the customer. Customer commitment in the face of project uncertainty is also fairly highly loaded into this factor (both load above .50). Finally technological uncertainty and co-location load in this factor above .40. The first factor therefore represents various concepts that measure a unique underlying 
construct. For the purpose of the current research, this factor was simply named empowerment of the project team.

The second factor emphasizes project criticality and project size, both of which load above .75 . These two variables represent the complexity of the project. Both of these variables had their scale reversed before the analysis. Therefore, all these complexity variables are measured from high complexity to low complexity, which is why the factor is named low project complexity. The last factor is chiefly loaded by the experience level of the team and consequently uses the same name (Table 4-20).

\begin{tabular}{lccc}
\hline \multicolumn{1}{c}{ Factors } & $\begin{array}{c}\text { P_FAC1 } \\
\text { Empowerment } \\
\text { of the project } \\
\text { team }\end{array}$ & $\begin{array}{c}\text { P_FAC2 } \\
\text { Low project } \\
\text { complexity }\end{array}$ & $\begin{array}{c}\text { P_FAC3 } \\
\text { of the project } \\
\text { team }\end{array}$ \\
\hline P1: Project criticality* & .195 & .785 & .056 \\
\hline P2: Experience level of the team & .068 & -.225 & .698 \\
\hline P3: Technological uncertainty & .461 & -.350 & -.396 \\
\hline P4: Project uncertainty & .582 & -.050 & -.398 \\
\hline P5: Procedural empowerment & .765 & -.051 & .022 \\
\hline P6: Customer commitment & .557 & .016 & .524 \\
\hline $\begin{array}{l}\text { P7: Methodology supported by the } \\
\text { customer (from plan-driven to agile) }\end{array}$ & .769 & .020 & .089 \\
\hline P8: Project size* & .139 & .754 & -.178 \\
\hline $\begin{array}{l}\text { P9: Co-location of the project team } \\
\text { members }\end{array}$ & .479 & .116 & .007 \\
\hline
\end{tabular}

* These scales were reversed for data analysis

Table 4-20: Factor analysis of the project variables

\subsubsection{Exploratory factor analysis of the SDM variables}

As expected, the factor analysis of the SDM variables revealed only one component, which explains $68.3 \%$ of the variability of the five SDM variables. This component gives approximately equal weight to all the variables. The loadings are represented in the table below (Table 4-21). SDM_FAC1 represents the SDM used on the project from plan-driven to agile. It was therefore named Agility. 


\begin{tabular}{lc}
\hline \multicolumn{1}{c}{ Factors } & $\begin{array}{c}\text { SDM_FAC1 } \\
\text { Variables }\end{array}$ \\
\hline SDM1.1:
\end{tabular}

Table 4-21: Factor analysis of the SDM variables

\subsubsection{Exploratory factor analysis of the project success variables}

A factor analysis showed that one component explains $61 \%$ of project success. This factor gives high loadings to all nine project success variables. Even PS3.2 with the lowest loading has a loading greater than .60. The new factor, PS_FAC1, was named Project Success (Table 4-22).

\begin{tabular}{lc}
\hline Variables & $\begin{array}{c}\text { PS_FAC1 } \\
\text { Project Success }\end{array}$ \\
\hline PS1.1: Time & .702 \\
PS1.2: Budget & .747 \\
PS1.3: Functionality & .824 \\
PS1.4: Quality & .885 \\
PS2.1: Address a need & .801 \\
\hline PS2.2: Product is used & .819 \\
PS2.3: Customer is satisfied & .850 \\
PS3.1: Team satisfaction & .759 \\
PS3.2: Team would work the same way & .601 \\
\hline
\end{tabular}

Table 4-22: Factor analysis of the project success variables

\subsubsection{Correlation analyses on the whole database}

Several correlation tests were first performed on the whole database to get a better understanding of the relationships between the variables and to evaluate the factors that most influence the selection of SDMs in practice, regardless of fit and success. These analyses do not provide findings that answer the research questions but they do provide information on the kind of data present in the database. 


\subsubsection{Correlation analysis among the independent variables}

First a correlation analysis was conducted between the independent variables (i.e. project and project environment). The findings are reported below (Table 4-23).

\begin{tabular}{|c|c|c|c|c|c|c|}
\hline & PE_FAC1 & PE_FAC2 & PE_FAC3 & P_FAC1 & P_FAC2 & P_FAC3 \\
\hline $\begin{array}{l}\text { PE_FAC1: Organizational } \\
\text { culture (from conservative to } \\
\text { entrepreneurial) }\end{array}$ & 1 & & & & & \\
\hline $\begin{array}{l}\text { PE_FAC2: Market } \\
\text { uncertainty }\end{array}$ & .000 & 1 & & & & \\
\hline $\begin{array}{l}\text { PE_FAC3: Low power } \\
\text { distance }\end{array}$ & .000 & .000 & 1 & & & \\
\hline $\begin{array}{l}\text { P_FAC1: Empowerment of } \\
\text { the project team }\end{array}$ & $.483^{* *}$ & .186 & .047 & 1 & & \\
\hline $\begin{array}{l}\text { P_FAC2: Low project } \\
\text { complexity }\end{array}$ & .048 & $-.305^{* *}$ & .067 & .000 & 1 & \\
\hline $\begin{array}{l}\text { P_FAC3: Experience level of } \\
\text { the team }\end{array}$ & .033 & -.046 & .033 & .000 & .000 & 1 \\
\hline
\end{tabular}

**. Correlation is significant at the .01 level (2-tailed).

Table 4-23: Correlation analysis between the independent variables

Two significant correlations between the independent factors are revealed by this test. The first one is between the organizational culture and empowerment of the project team. Thus in organizations with an entrepreneurial culture, project teams tend to be more empowered; while in organizations with a conservative culture, project teams tend to be less empowered.

The next significant correlation is between the complexity of the project and market uncertainty. This means that when market uncertainty is high, projects are usually more complex; and when market uncertainty is low, projects are usually less complex.

Another correlation test performed on all the variables (PE1 to PE5, P1 to P9 and DEM2, instead of the factors) highlighted the same relationships. In addition, the organization size is significantly correlated with the project management methodology $(r=.333, p<.01)$, the level of entrepreneurship $(r=-.314, p<.01)$ and the level of risk-aversion $(r=.295, p<.01)$. This indicates that the bigger the 
organization, the less entrepreneurial they are and the more they use a plan-driven approach.

There is also a significant correlation between the methodology favoured by the customers and their level of commitment to the project $(r=.462, p<.01)$. In other words, customers who favour an agile approach are more willing to commit themselves to the project.

Finally, a correlation was found between project uncertainty and market uncertainty $(r=.292, p<.01)$.

\subsubsection{Correlation analysis between the independent variables and SDM}

A correlation analysis on the whole database between the independent variables and the SDM (from plan-driven to agile) was then conducted. It shows the variables that significantly correlate with the choice of the methodology selected. These findings, however, do not show which variables are important in SDM fit, but only those that have the most correlation with the SDM choice in practice. The variables that significantly correlate with SDM are in bold in the table below (Table 4-24).

\begin{tabular}{lll}
\hline Factors & $\begin{array}{l}\text { Correlation } \\
\text { coefficients }\end{array}$ & $\begin{array}{l}\text { Significance } \\
\text { (1-tailed) }\end{array}$ \\
\hline $\begin{array}{l}\text { PE_FAC1: Organizational culture } \\
\text { (from conservative to entrepreneurial) }\end{array}$ & $.561^{* *}$ & .000 \\
\hline PE_FAC2: Market uncertainty & .039 & .347 \\
PE_FAC3: Low power distance & .082 & .204 \\
\hline P_FAC1: Empowerment of the project team & $.663^{* *}$ & .000 \\
\hline P_FAC2: Low project complexity & .084 & .207 \\
\hline P_FAC3: Experience level of the team & .040 & .350 \\
\hline
\end{tabular}

**. Correlation is significant at the .01 level (1-tailed).

Table 4-24: Correlation analysis between the independent factors and SDM

In practice, two factors correlate with the choice of a SDM: organizational culture and empowerment of the project team.

In addition, a correlation analysis with all of the original independent variables instead of the factors confirms these findings. The SDM seems to be most influenced 
by what approach top management and customers support. A significant negative correlation $(r=-.189, p=.026)$ was also found between the organization size and the SDM utilized. The polarity of the variables is such that a large organization is associated with a less agile approach, and a small organization is associated with a more agile approach.

\subsubsection{Analysis of the SDM fit model}

This analysis aims at finding a model of SDM fit as well as determining what the key factors in SDM fit are. It is conducted on successful projects only (PS_FAC1 > 0) as it is considered that those projects present a high level of SDM fit. The correlations reported are therefore not between an independent variable and SDM, but between an independent variable and an SDM that fits. The number of projects considered successful is 46 out of the total of 106 projects that were included in the database.

\subsubsection{Correlation analysis}

The test was 1-tailed because the relationship between the independent factors and the SDM (from plan-driven to agile) is predicted to be in a particular direction.

\begin{tabular}{lll}
\hline Variables & $\begin{array}{l}\text { Correlation } \\
\text { coefficients }\end{array}$ & $\begin{array}{l}\text { Significance } \\
\text { (1-tailed) }\end{array}$ \\
\hline $\begin{array}{l}\text { PE_FAC1: Organizational culture } \\
\text { (from conservative to entrepreneurial) }\end{array}$ & $.514^{* *}$ & .000 \\
\hline PE_FAC2: Market uncertainty & .235 & .056 \\
\hline PE_FAC3: Low power distance & .031 & .417 \\
\hline P_FAC1: Empowerment of the project team & $.802^{* *}$ & .000 \\
\hline P_FAC2: Low project complexity & .117 & .216 \\
\hline P_FAC3: Experience level of the team & .117 & .216 \\
\hline **.COrrelation is significant at the 01 level (1-tailed) &
\end{tabular}

**. Correlation is significant at the .01 level (1-tailed).

Table 4-25: Correlations of the independent factors with SDM (SDM fit research model)

Table 4-26 exhibits three correlations between an independent variable and an SDM that fits. Firstly, empowerment of the project team is strongly and positively correlated with an SDM that fits. An empowered project team is associated with an 
agile SDM that fits, and an un-empowered project team is associated with a plandriven SDM that fits.

Secondly, organizational culture is strongly correlated with an SDM that fits. An entrepreneurial culture is correlated with an agile SDM that fits, while a conservative culture is correlated with a plan-driven SDM that fits.

Thirdly, market uncertainty is negatively correlated with choice of an SDM that fits. An uncertain market is correlated with an agile SDM that fits, and a certain market is correlated with a plan-driven SDM that fits. However the significance value of $p=$ .056 , while less than the value $(p=.01)$ sometimes adopted for exploratory research, is slightly greater than the .05 threshold adopted by this study. Therefore it cannot be concluded that there is a significant correlation between market uncertainty and an SDM that fits.

In summary, correlations exist between an SDM that fits and two factors derived from the independent variables:

Project environment factors:

- Organizational culture. This factor includes three project environment variables: the methodology supported by top management, level of entrepreneurship, and level of risk-taking willingness

Project factors:

- Empowerment of the project team. This factor includes six project variables: methodology supported by the customer, procedural empowerment, project uncertainty, customer commitment, co-location of the project team members, and technological uncertainty.

On the other hand, the following factors do not significantly account for nor contribute to variability in SDM fit, and therefore may have limited impact of SDM fit:

Project environment factors:

- Market uncertainty $(\mathrm{p}=0.56)$

- Power distance (few responses were received from other than low power distance countries). 
Project factors:

- Complexity. This factor includes 2 project variables: project criticality, and project size

- Experience level of the team.

Therefore, correlation analysis of the SDM fit model provides support for the following hypotheses:

H1: Project environment factors influence the choice of SDM that fits.

\section{Supported}

H2: Project factors influence the choice of SDM that fits. Supported

In brief, both the project characteristics and the project environment have an impact on the selection of the SDM that fits.

Interestingly, organization size, which is correlated with SDM (per the test on the whole database), is not correlated with an SDM that fits (per the test on successful projects).

Project management methodology was subject to a separate correlation analysis. It is expected to span both project environment and project, but is not expected to be strongly associated with particular variables or strongly load on factors derived from each. Project management methodology was not included in the hypotheses and indeed does not appear in any factor. However it shows a very strong correlation $(r=$ .905 and $p=.000$ ) with an SDM that fits, indicating respondent belief that the alignment between the project management methodology level and SDM that fits is important to achieving SDM fit.

\subsubsection{Multiple regression analysis}

The multiple regression analysis explores the causal relationships between multiple independent variables and a dependent variable. This method delivers the relative 
predictive importance of the independent variables and an overall measure of predictive power $\mathrm{R}^{2}$.

To determine the best predictors of SDM, a regression analysis on SDM_FAC1 was then conducted. The factors identified by the correlation analysis (Table 4-25) were selected as candidates for the regression analysis.

P_FAC1 (empowerment of the project team) and PE_FAC1 (organizational culture) are the two predicting factors of SDM_FAC1 for successful projects (Table 4-26).

\begin{tabular}{|c|c|c|c|c|c|}
\hline & \multicolumn{2}{|c|}{ Unstandardized Coefficients } & \multicolumn{3}{|l|}{$\begin{array}{l}\text { Standardized } \\
\text { Coefficients }\end{array}$} \\
\hline & B & Std. Error & Beta & $\mathrm{t}$ & Sig. \\
\hline Constant & .001 & .073 & & .021 & .984 \\
\hline $\begin{array}{l}\text { P_FAC1: empowerment of } \\
\text { the project team }\end{array}$ & .515 & .084 & .508 & 6.110 & .000 \\
\hline $\begin{array}{l}\text { PE_FAC1: organizational } \\
\text { culture }\end{array}$ & .311 & .082 & .315 & 3.782 & .000 \\
\hline
\end{tabular}

Table 4-26: Regression coefficients (SDM fit research model)

Both factors have a significance value (i.e. .000) that is less than .05, which indicates that they are good, significant predictors of SDM_FAC1. This model, that contains two predictors, explains more than 50\% of the variability of the SDM (Table 4-27).

\begin{tabular}{llll}
\hline $\mathbf{R}$ & R Square & $\begin{array}{l}\text { Adjusted R } \\
\text { Square }\end{array}$ & $\begin{array}{l}\text { Std. Error of the } \\
\text { Estimate }\end{array}$ \\
\hline .715 & .512 & .501 & .667 \\
\hline
\end{tabular}

Predictors: Constant, P_FAC1. Dependent Variable: SDM_FAC1

Table 4-27: Regression model summary (SDM fit research model)

Based on the standardized coefficients, the model of SDM fit can be summarized by the following equation:

$$
S D M \_F A C 1=.508 P \_F A C 1+.315 P E \_F A C 1
$$

A unit increase in empowerment of the project team produces a .508 increase in the measure/score for SDM that fits. Similarly, a unit increase in organizational culture produces a .315 increase in the measure/score for SDM that fits. 
The regression model is represented in the figure below. The standardized coefficients are reported in Figure 4-1.

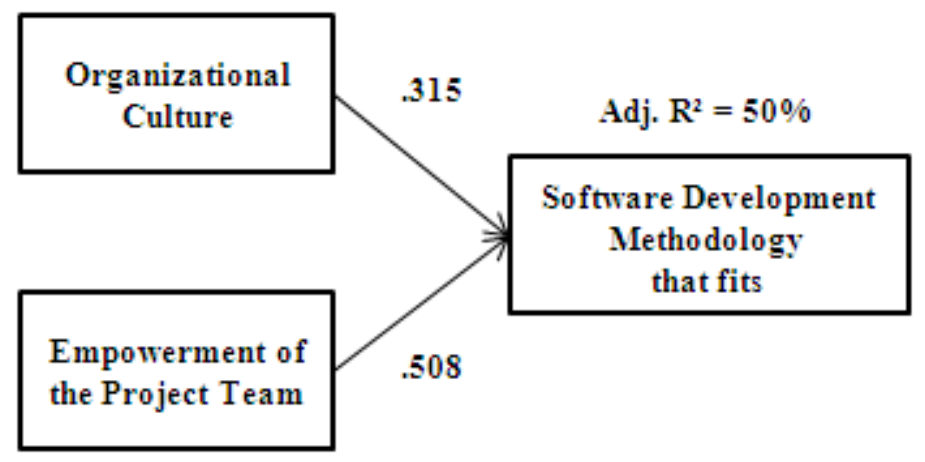

Figure 4-1: Regression analysis summary (SDM fit research model)

To conclude the analysis of the first part of the model, P_FAC1 (empowerment of the project team) and PE_FAC1 (organizational culture) are both contingency factors in SDM selection. While the appropriate statistical analysis has yet to be performed, the regression coefficients suggest that that P_FAC1 (.508) may prove a more influential measure than PE_FAC1 (.315).

\subsubsection{Analysis of the project success model}

This analysis determines the importance of project environment, SDM fit and project on project success. For this analysis, SDM fit is when the SDM matches what the model of fit presented above predicts. Thus a measure of fit was derived by applying the model of SDM fit developed above to all the projects and comparing SDM (the methodology used) and SDM that fits (the methodology predicted).

\subsubsection{Correlation analysis}

First, a correlation analysis was conducted between project success (PS_FAC1) and the independent factors presented in the project success research model.

This test could have arguably been conducted with a 1-tailed significance for certain of the variables, including SDM fit for example. However, the results of the more conservative 2-tailed significance test are reported for all factors. 
This test results reported in Table 4-28 exhibit three significant correlations with project success. The first involves the experience level of the project team. When more experienced teams work on a project, the project is more likely to be successful. Secondly, projects conducted in an environment where market uncertainty is low are more likely to be successful. Thirdly, and more importantly, SDM fit is significantly and positively correlated with measures of project success, and makes a statistically significant contribution to measures of project success, thus supporting our hypotheses that SDM fit has impact on project success.

\begin{tabular}{lll}
\hline & $\begin{array}{l}\text { Correlation } \\
\text { coefficients }\end{array}$ & $\begin{array}{l}\text { Significance } \\
\text { (2-tailed) }\end{array}$ \\
\hline $\begin{array}{l}\text { PE_FAC1: Organizational culture } \\
\text { (from conservative to entrepreneurial) }\end{array}$ & .092 & .379 \\
PE_FAC2: Market uncertainty & $-.246^{*}$ & .017 \\
PE_FAC3: Low Power distance & .136 & .193 \\
FIT: SDM fit & $.216^{*}$ & .042 \\
\hline P_FAC1: Empowerment of the project team & -.038 & .725 \\
P_FAC2: Low complexity & .118 & .272 \\
\hline P_FAC3: Experience level of the team & $.365^{* *}$ & .000 \\
\hline *. Correlation is significant at the .05 level (2-tailed). \\
**. Correlation is significant at the .01 level (2-tailed).
\end{tabular}

Table 4-28: Correlations with project success (project success research model)

Therefore support has been found for the following hypotheses:

\section{H3: Project environment factors influence project success. Supported \\ H4: SDM fit influences project success. Supported \\ H5: Project factors influence project success. Supported}

Project environment, SDM fit, and project all have an impact on project success. Nevertheless, as shown above, all the variables included in these clusters do not have an influence on project success. The project environment factor that influences project success is market uncertainty and the project factor that influences project success is the experience level of the team.

In addition, a correlation analysis conducted with the original variables instead of the factors showed that technological and project uncertainty are also significantly 
negatively correlated with project success (respectively $r=-.222$, $p=.031$ and $r=-.295$ and $p=.004)$. Thus the more stable the project is in terms of market, technology and project requirements, the more likely it is to be successful.

Finally, a correlation analysis was conducted between measures of SDM (not SDM fit) and project success to evaluate if one approach delivers more project success than the other in all situations. In other words, it tested whether one size fits all. No correlation was found between these two factors $(r=.165, p=.110)$, which confirms that one size does not fit all. The use of one particular approach regardless of the project and its environment will not increase project success.

\subsubsection{Multiple regression analysis}

A stepwise linear regression confirmed the importance of three previously identified factors in predicting project success. A stepwise regression procedure was applied to find the model that best predicted the dependent variable, that is, the model with the highest $\mathrm{R}$ square. The stepwise procedure only enters one independent variable at a time in the model, until there is no remaining variable. Only the variables that significantly contribute to the prediction of the dependent variable $(p<.05)$ are selected to be entered in the model, while others are excluded. The experience level of the project team is significantly correlated with the project success, and its associated regression Beta coefficient signals a higher contribution to measures of project success, and therefore to predicted levels of success than do other variables (Table 4-29).

\begin{tabular}{|c|c|c|c|c|c|}
\hline & \multicolumn{2}{|c|}{ Unstandardized Coefficients } & \multirow{2}{*}{\multicolumn{3}{|c|}{$\begin{array}{l}\text { Standardized } \\
\text { Coefficients }\end{array}$}} \\
\hline & & & & & \\
\hline & B & Std. Error & Beta & $\mathbf{t}$ & Sig. \\
\hline Constant & .243 & .169 & & 1.442 & .153 \\
\hline $\begin{array}{l}\text { P_FAC3: Experience level of the } \\
\text { team }\end{array}$ & .391 & .100 & .376 & 3.929 & .000 \\
\hline PE_FAC2: Market uncertainty & -.217 & -0.96 & -.215 & -2.245 & .027 \\
\hline FIT: SDM fit & .303 & -142 & .204 & -2.135 & .036 \\
\hline
\end{tabular}

Table 4-29: Regression coefficients (project success research model)

This model of project success can be summarized by the following equation. 


$$
P S \_F A C 1=.376 P \_F A C 3-.215 \text { PE_FAC2 }+.204 \text { FIT }
$$

A unit increase in experience level of the team produces a .376 unit increase in project success A unit increase in market uncertainty produces a .215 unit decrease in project success. Finally, a unit increase in SDM fit produces a .204 unit increases in project success.

This model, that contains three predictors, explains almost a quarter of the variability in measures of project success (Table 4-30).

\begin{tabular}{llll}
\hline $\mathbf{R}$ & R Square & $\begin{array}{l}\text { Adjusted R } \\
\text { Square }\end{array}$ & $\begin{array}{l}\text { Std. Error of the } \\
\text { Estimate }\end{array}$ \\
\hline .491 & .242 & .214 & .902 \\
\hline
\end{tabular}

Predictors: Constant, P_FAC3, PE_FAC2, and FIT. Dependent Variable: PS_FAC1

Table 4-30: Regression model summary (project success research model)

The regression model can be summarized as follows (Figure 4-2):

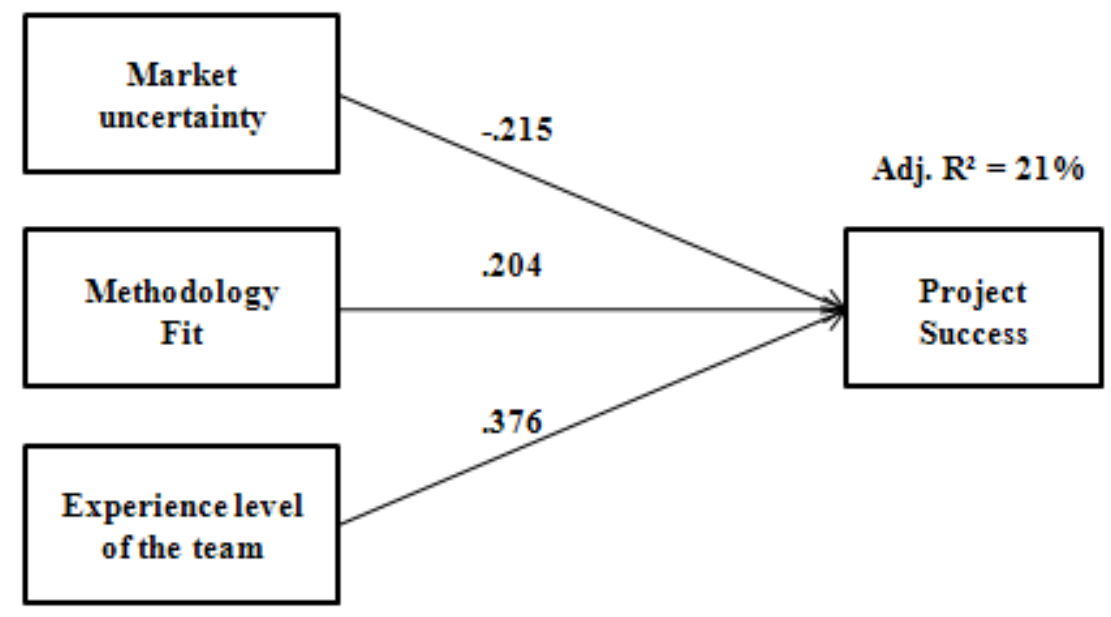

Figure 4-2: Regression analysis summary (project success research model)

\subsubsection{Limitations of data analysis and validity}

The exploratory factor analysis, correlation and regression tests used to conduct the data analysis have their limitations and one could argue that a structural equation modelling (SEM) approach would have been more appropriate. However, this is an exploratory study in which many constructs are measured by a single variable, a situation not very well suited for SEM. In addition, SEM employs confirmatory 
factor analysis, which requires theoretical support to justify the relationships in the SEM model between variables and factors. Because this research is exploratory, there is no support for a confirmatory factor analysis on the independent variables.

Internal validity was addressed by the use of questions from existing instruments whenever possible, but strong internal validity for this study cannot be claimed as this study is exploratory. External validity was addressed by a relatively large sample. However, there is a possible bias due to the overrepresentation of three countries in the sample. A larger, random sample could be employed in future research to improve external validity.

\subsection{Conclusion}

Survey data analysis showed that empowerment of the project team and organizational culture are the two key contingency factors in SDM fit. These findings concur with the results of analysis of interview data which showed that their constituent variables were regarded as highly important by project managers when selecting a SDM.

Further, this study shows that one size does not fit all. The choice of a particular SDM, regardless of the project and project environment, does not improve project success. On the other hand, SDM fit was found to have an impact on project success, along with the experience level of the team and market uncertainty.

These findings are interpreted and discussed in the next chapter. 


\section{CHAPTER 5: DISCUSSION}

The fifth chapter interprets and discusses the data analysis presented in the previous chapter by using logical justification, previously published literature, and responses obtained through the open-ended survey question at the end of the survey. Each factor is discussed before explaining the implication for project management researchers and practitioners.

\subsection{Project Environment}

This first cluster contains the factors representing the environment of the project. This dimension has often been excluded from studies on SDM fit (for example, Burns \& Dennis, 1985; Boehm \& Turner, 2003). Although considered as less important than project factors by researchers, the current study shows that some of the factors in this category play a critical role in SDM fit. In particular, integrating agile approaches and philosophies into more conservative project environment seems difficult. The project environment factors and their associated variables (Table 5-1) are discussed in the next paragraphs. An operational definition of all the variables can be found in Appendix F.

\begin{tabular}{llll}
\hline $\begin{array}{l}\text { Factor 1: } \\
\text { Organizational culture }\end{array}$ & $\begin{array}{l}\text { Factor 2: Market } \\
\text { Uncertainty }\end{array}$ & $\begin{array}{l}\text { Factor 3: Low Power } \\
\text { Distance }\end{array}$ & $\begin{array}{l}\text { Other Project } \\
\text { Environment Factors }\end{array}$ \\
\hline $\begin{array}{l}\text { Methodology } \\
\text { supported by top } \\
\text { management }\end{array}$ & Market uncertainty & Power distance & $\begin{array}{l}\text { Project management } \\
\text { methodology }\end{array}$ \\
$\begin{array}{l}\text { Level of } \\
\text { entrepreneurship }\end{array}$ & & Economic sector \\
$\begin{array}{l}\text { Level of risk-taking } \\
\text { willingness }\end{array}$ & & Organization size \\
\hline
\end{tabular}

Table 5-1: Project environment factors and their associated variables

\subsubsection{Organizational culture}

The organizational culture varies from conservative cultures (at the low-end of the scale) for plan-driven methodologies to entrepreneurial cultures (at the high-end of the scale) for agile methodologies. In the current study, organizational culture is a 
function of the level of entrepreneurship and risk-taking willingness of the organization, as well as the methodology supported by top management. Actually, executive support is reflected in the culture of the organization. In conservative organizations, executives prefer more traditional approaches and are less willing to take risks.

The current study found significant statistical support for the correlation between organizational culture and SDM fit (see section 4.2.7.1). This finding is consistent with Strode et al. (2009) who found a relationship between low formality organizations and the use of agile methodologies. It is also consistent with Iivari and Huisman (2007), who found a positive relationship between the hierarchical rational organizations and the deployment of traditional methodologies.

One aspect of the organizational culture is the way the organization responds to change. An organization that is responsive to change will easily adapt to the agile style of software development, which is based on flexibility and responsiveness. On the other hand, a rigid organization will feel more comfortable with a similar style of development, that is, a traditional approach. In summary, an entrepreneurial, agilefriendly environment is a critical factor in the choice of an agile approach (as in Chow \& Cao, 2008), while a conservative environment is important in the choice of a traditional approach.

Organizational culture has an important impact on how the business is run and particularly on the way projects are managed. Nerur, Mahapatra, and Mangalaraj (2005) explain that the organizational culture has an impact on the behaviour and action of people. This is confirmed by the current study, which shows that the organizational culture impacts on the level of team members' empowerment. In other words, the environment in which the project is conducted influences the empowerment and the culture of the project team.

Culture has many facets but this study only focuses on some of them. Although culture can be changed, such change is slow and requires a long term effort (Koch, 2005). Thus this study considers that culture, reflected in people's mindsets, is static and cannot be easily changed to suit a particular project. That is why this factor is 
often described as one of the main challenges in adopting agile methodologies (Nerur et al., 2005). The three dimensions included in this factor are discussed next.

\subsubsection{Methodology supported by top management}

Executive support is cited in the Standish Group report (2001) as the most influential factor on project success. This study found significant statistical support for the correlation between top management support for a particular approach (from plandriven to agile) and SDM fit. This finding provides statistical support for the commonsense idea that the approach favoured by executives influences the way the whole project is conducted.

The influence of top management can be explained by the power they have over the project. They may, for example, decide to terminate a project at any time or give it more resources.

In both the interviews and the survey, this variable sparked off many reactions about the politics involved in a project. For example, a project manager explained he was forced to abandon the scrum methodology on his project and replace it by a plandriven approach for political reasons, though the project was showing signs of success. The consequence was a failure to deliver anything. Executives had to recognize their 'mistake' and, because of the lack of results, again allow the use of scrum. Another project manager explained that the implementation of certain agile principles was not allowed in his project, which he claims undermined the success of his project.

Consequently, executive support is essential and getting the buy-in of top management for an approach is important in project success. This factor should therefore not be neglected. In many cases however, instead of the project manager, it is the executives who choose which methodology will be used on the project.

This factor may, in certain cases, be used as a variable of adjustment by project managers who wish to implement a certain methodology. Supporting agile methodologies is, however, a major cultural shift for traditional executives, who want to know prior to their own commitment, the delivery dates, cost, and 
functionality that will be delivered. With agile methodologies, they have little critical information about such factors. Thus efforts may need to be spent by the project manager before the beginning of the project to generate executive buy-in to the most appropriate SDM for a particular project. In that regard, a decision-tool based on the factors identified in this study may be helpful.

\subsubsection{Level of entrepreneurship}

The level of entrepreneurship is one of the many dimensions representing the culture of the organization. Highsmith (2002) explained that agile methodologies thrive better in leadership and collaboration cultures whereas traditional methodologies thrive better in command and control cultures. It seems very difficult for agile people to perform well in rigid or conservative organizations. This claim is supported by the current study which found significant statistical correlation between level of entrepreneurship and SDM fit.

Although previous researchers have not used the level of entrepreneurship as a contingency factor, this study shows that it plays an essential role. Agile methodologies fit entrepreneurial organizations, while traditional methodologies perform better in a conservative environment where there are usually many control procedures in place.

\subsubsection{Level of risk-taking willingness}

The current study found that the level of entrepreneurship is significantly and positively correlated with level of risk-taking willingness. This supports the literature in Table 2-4 which treats the two variables as closely related. Level of risk-taking willingness represents the willingness of organizations to thrive on chaos rather than on order. Agile approaches require the organization to be willing to take some risks as there is more uncertainty related to agile projects than plan driven projects. Thus organizations that are willing to take risks are able to better accept project uncertainty and iterations provided by agile methodologies. 
On the other hand, organizations that are risk-averse want a detailed plan and schedule of the whole project to avoid any unexpected and detrimental events. This level of comfort is provided by plan-driven methodologies which plan up-front for the whole project. In some public organizations, the level of risk-aversion may be so sufficiently high to cause major delays the beginning of the project. For instance, an interviewee explained that in his organization, it may take up to several years to get the project and plan accepted by executives because his organization does not want to take any risk.

\subsubsection{Market uncertainty}

This factor has been cited as one of the sources of uncertainty by several authors (Highsmith, 2010; Pixton et al., 2009; Wysocki, 2009), who believe that it influences the choice of a methodology. In the current study however, it cannot be concluded that this factor plays any significant role in methodology fit (the significance value $p$ is slightly higher than .05 , see Table $4-25)$. In the database, there are many examples of successful linear projects with a market uncertainty ranging from very stable to very unstable. Similarly, there are many successful agile projects with a market uncertainty ranging from low to high.

However as reported in section 4.2.6.2, this factor is correlated with project uncertainty (i.e., requirement stability) which is one of the key contingency factors.

\subsubsection{Power distance}

Power distance partly reflects the national culture and its influence on how the project is conducted. Misra et al. (2009) found that the societal culture factor had a significant bearing on agile project success. This factor, however, was not measured using the power distance index of Hofstede employed by the current study.

The findings of the current research do not show that this factor has any statistically significant association with or observable impact on SDM fit. Although some countries with a very high power distance were represented in the database, most countries had a low or very low power distance, which makes the sample unsuitable 
for testing related theory. In fact, nearly $70 \%$ of the respondents were from three countries (USA, Australia and New Zealand). Consequently, we cannot draw any conclusions on this factor, and further research is needed.

\subsubsection{Other project environment variables}

Several other project environment variables were considered by the current research. They are discussed below.

\subsubsection{Project management methodology}

The software development methodology (measured on a spectrum from plan-driven to agile) was highly correlated with the project management methodology (for example, PRINCE2, PMI...) stated by the respondents (see section 4.2.7.1). This suggests that to achieve SDM fit, a strong alignment between the project management and software development levels is needed (see Figure 2-1). This finding is drawn from 81 of the respondents (76\%) as 25 of the projects (24\%) were managed with an in-house methodology. As participants did not describe the inhouse methodology, it was not possible to evaluate what kind of methodology it was.

PRINCE2, a prescriptive project management methodology, was only used on plandriven development projects, which supports the interviewees' contention that it is too prescriptive to be combined with agile SDMs. PMI (PMBOK), which is a descriptive, rather than prescriptive, project management methodology, was successfully used on both plan-driven and agile projects.

Although project management methodology is correlated with SDM fit this factor is important in SDM fit, it cannot be used to decide which SDM should be used. It should rather be selected or adapted to match the project needs in the same manner as the SDM is selected. Therefore, the choice of both the project management and SDMs should be made at the same time to ensure that they are compatible. It is particularly important for organizations to make sure that these methodologies are aligned, so that information can flow well in both directions between them. 


\subsubsection{Economic sector}

Projects from many different industries (see Table 4-14) were analysed. Only 11 of them were conducted in the government sector while the rest (i.e. 95) was conducted in private organizations.

The public sector is traditionally known to be conservative and risk-averse, which is an indicator of plan-driven approaches. Out of the 11 projects conducted in the public sector, seven were managed with a traditional methodology. An agile methodology was used on the four other projects. Interviews and surveys both showed that agile methodologies are not incompatible with at least some public projects but must be carefully tailored to suit the organizational environment.

Public organizations in the studied sample were very risk-averse (scoring 4 out of 5 on average) and not very entrepreneurial (scoring 2 out of 5 on average), which confirms the interviewees' assertions. The number of public organizations in the sample is, however, too small to draw any conclusions regarding that factor. However, the public and private sectors differ in their level of entrepreneurship and risk-aversion, which were found to be significant in SDM fit.

\subsubsection{Organization size}

In the current study, the analysis of survey current study found no significant statistical correlation between organization size and SDM fit. This supports the views of interviewees that organization size plays no significant role in SDM fit. The analysis on the whole survey dataset revealed that although it is not a contingency factor in SDM fit, it is significantly correlated to the SDM in practice. It means that, in practice, bigger organizations tend to choose more prescriptive approaches, and smaller organizations (often more entrepreneurial) prefer agile methodologies. As bigger organizations have more procedures and processes in place, this naturally leads them towards traditional approaches. However, the size of the organization should not be considered as an important factor when selecting a methodology. Other more important project environment factors like the level of entrepreneurship should be considered instead. 


\subsection{Project}

Project factors have attracted the most attention of researchers. For example, Boehm and Turner (2003) focused on the home ground of project characteristics within which each methodology performs very well, without considering the project environment. However, the current study found statistical correlations that support only two of their five critical project variables (namely, project uncertainty and procedural empowerment). These factors are presented as requirements stability and control culture in Figure 2-7.

As expected from what was revealed in the interviews, most of the variables in this cluster were found to be significantly correlated with SDM fit. A summary of the project factors and the associated variables measured in this study is provided in the table below (Table 5-2). An operational definition of these variables can be found in Appendix F.

\begin{tabular}{lll}
\hline $\begin{array}{l}\text { Factor 1: } \\
\text { Empowerment of the Project Team }\end{array}$ & $\begin{array}{l}\text { Factor 2: } \\
\text { Complexity }\end{array}$ & $\begin{array}{l}\text { Factor 3: } \\
\text { Experience Level of the Team }\end{array}$ \\
\hline $\begin{array}{l}\text { Technological uncertainty } \\
\text { Project uncertainty }\end{array}$ & Project size & Experience level of the team \\
\hline $\begin{array}{l}\text { Procedural empowerment } \\
\text { Customer commitment }\end{array}$ & \\
\hline $\begin{array}{l}\text { Customer support } \\
\text { Co-location of the project team } \\
\text { members }\end{array}$ & \\
\hline
\end{tabular}

Table 5-2: Project factors and their associated variables

All these factors and variables are discussed below.

\subsubsection{Empowerment of the project team}

This first factor, which encapsulates two of the five project factors included in Boehm and Turner's model (2003), is clearly an important factor in SDM fit (see Figure 4-1). It includes various variables, which are discussed below. 


\subsubsection{Technological uncertainty}

On the average, those who responded to the survey were experienced and perceived low levels of technological uncertainty (Table 4-16). Nevertheless, the analysis of survey data revealed that respondents perceived technological uncertainty to be significantly and positively correlated with SDM. The latter finding is consistent with the literature summarised in Table 2-4 which identifies technological uncertainty as an important factor in SDM fit (e.g., see Tortamış, 2004). It is a potential source of change or risk during the software development process, which is a key discriminator of traditional SDMs. A well-known technology is unlikely to be a major source of change. Therefore, it is suitable for traditional methodologies. On the other hand, a new technology may not go as planned and will require the project team to adapt as they learn. This gradual learning is suitable for agile approaches as they use short iterations and constant feedback.

\subsubsection{Project uncertainty}

This factor is related to the ability to understand the requirements in advance and the probability that the requirements will change during the development process. It also depends on the client's ability to correctly communicate the system functionalities or requirements. The analysis of survey data revealed that respondents perceived project uncertainty to be significantly and positively correlated with SDM.

One of the most critical differences between traditional and agile approaches is the way they handle change. The traditional approach attempts to minimize change, while the agile approach embraces it. Traditional methodologies predict and control while agile methods adapt and innovate (Vinekar, Slinkman, \& Nerur, 2006). Responding to change is one of the key principles of the agile manifesto. Change can sometimes happen faster than a plan can be changed, which is the kind of situation that agile methodologies handle very well. Thus, as suggested by many researchers such as Boehm and Turner (2003), this study demonstrates that project uncertainty has an essential role in methodology selection and fit. This factor was already found 
to be a contingency factor between prototyping and traditional methodology by Burns and Dennis (1985).

As reflected in the content of Table 2-1, agile SDMs were developed to respond to change and uncertainty in requirements and to reduce the cost of change throughout the project (Cockburn \& Highsmith, 2001). The current study provides statistically significant findings that this is indeed the case. Agile methodologies accommodate change by employing a rapid iterative development process. In addition, they first implement features that deliver the most value. As a result, if this principle is correctly followed and if the customer is knowledgeable and committed, project uncertainty should not be a major issue. On the other hand, traditional approaches for projects with low uncertainty have the advantage of optimizing the development process.

Although project uncertainty is important in SDM selection, the team has to find the right balance when responding to change. An interviewee agreed with Boehm's statement (2002) that over-responding to change can be costly and may in certain cases lead to project failure.

\subsubsection{Procedural empowerment}

Procedural empowerment was briefly discussed in section 4.1.3.4 (Culture of the project team). Both the interview and survey findings support the idea that empowerment is an important factor in the project category. This important aspect of the project team culture was already emphasized by the literature (Boehm \& Turner, 2003; Misra et al., 2009).

It is essential for the successful implementation of agile methodologies that project team members are empowered and most importantly that they feel comfortable being so. If they do not feel comfortable that way or if it contradicts the organizational culture, projects may not be better managed by an agile approach. In other words, project team members' culture has to match the level of procedural empowerment experienced by team members. Without project team members' procedural empowerment, many agile methodologies cannot be successfully implemented. 
While procedural empowerment is important in both traditional and agile projects, it is more important in agile projects. That is, compared to a traditional project, an agile project is more likely to fail in the absence of procedural empowerment, and more likely to succeed in the presence of procedural empowerment.

Besides, the selection of a SDM does not only have an impact on the level of empowerment of the project team members but also on the decision-making authority. The latter should be located at different levels according to the approach chosen. In agile organizations, executives must be willing to share decision-making authority. While top management remains accountable for business-level decisions, team members take accountability for all the technical issues (Williams \& Cockburn, 2003). On the other hand, with traditional approaches, the decision-making authority belongs to the project board. This is one of the main reasons why it is often a challenge for traditional organizations to move to agile methodologies.

In summary, this factor should be carefully considered when selecting a methodology. Not only should the right level of empowerment be given to the team, but the team members should also feel comfortable being empowered or following pre-established procedures. This factor can be adjusted by selecting different team members when possible to better match the methodology chosen.

\subsubsection{Customer commitment}

Customer commitment, also referred to as customer collaboration or customer involvement, is the level to which the customer is willing to invest in the development process. This factor is not only one of the agile manifesto principles, it is also considered to be one of the most important contingency factors. If the customer does not commit himself to the project, agile methodologies are unlikely to succeed, and plan-driven methodologies will be more appropriate as they require little customer involvement after the initial specification phase. In the current study, the analysis of survey data revealed that respondents perceived customer commitment to be significantly and positively correlated with SDM. 
Thus while the involvement of the customer in the development process is an important characteristic of agile methodologies (Cockburn \& Highsmith, 2001), it is less a problem for traditional SDMs. The latter actually depend on the ability of the customer to specify the full requirements up-front. If the customer is not able to articulate his needs clearly, this can threaten the whole project.

One of the survey participants mentioned a particular agile project, which was successful though stressful for the development team. The customer was not fully committed to the project and could not make up his mind about the requirements and their priority. Similar examples were given by interviewees. The examples align with the idea that in agile projects the customer should not only be committed but also knowledgeable, representative, and empowered, as discussed by Boehm (2002). As explained by Tortamış (2004), without a good level of commitment from the customer, agile projects will suffer and may fail. This entails an important amount of responsibility on the customer or its representative, which he may be unwilling to take on.

Many agile methodologies recommend a full-time customer presence on site, working with the project team. However, such a person may not be available, which can prevent the implementation of agile projects (Highsmith, 2004). Further, the issue of customer qualification has not been addressed by this study although it may be an equally important factor that would need to be investigated in further research.

In brief, the availability of customer representatives must be considered when deciding between agile and traditional SDMs. If the customer is able to provide an on-site representative who is knowledgeable and empowered, then agile methodologies are most suitable. On the other hand, if the client can only give a formal set of requirements at the beginning of the project, then a traditional approach is more appropriate. This finding support previous studies such as Chow and Cao (2008) and Vinekar et al. (2006). 


\subsubsection{Methodology supported by the customer}

Similarly to top management support, user or customer support for the chosen development approach is essential. In the current study, the analysis of survey data revealed that respondents perceived the methodology supported by the customer to be significantly and positively correlated with SDM.

This factor is closely related to the customer's culture (Vinekar et al., 2006). Indeed, a customer who is uncomfortable with flexible budgets and schedules, and prefers specific features, deadlines, and costs, is unlikely to support agile methodologies. Another reason why a customer would not support an agile approach is because he does not want to or cannot commit a person full-time to the project. On the contrary, if the customer organization has an adaptive, flexible, and entrepreneurial culture, he may not want to support a rigid traditional approach that requires up-front detailed and formal specification.

The survey comments provided several examples of clients suspicious of agile methodology because of its apparent lack of discipline. Most of them eventually bought into agile having seen good outcomes. Thus, even though it is an important factor in methodology, customer support can be altered to match the methodology chosen by the project team.

\subsubsection{Co-location of the project team members}

In the current study, the analysis of survey data revealed that respondents perceived the methodology supported by the customer to be significantly and positively correlated with SDM. This statistical result supports the claim that co-location of the project team members influences the choice of the most appropriate methodology. Some authors consider co-location to be essential in agile methodologies to reduce the cost of moving information between people and thus being able to respond more effectively to change (Cockburn \& Highsmith, 2001).

In the case of big projects, co-location is not always possible. The Forrester report (West \& Grant, 2010) explains that dividing labour within projects is one of the 
means to overcome that potential obstacle when an agile approach is chosen. Thus, the project is divided in smaller projects and a release team then aggregate the work of all the sub-teams. This is a sort of "scrum of scrum", where the members of a subteam are co-located, but overall all the project members do not need to be in the same place.

\subsubsection{Complexity}

This factor, which encapsulates two of the five factors included in Boehm and Turner's model (2003), was not found to be significant in SDM fit. Findings about these two factors (project criticality and project size) are briefly discussed below.

\subsubsection{Project criticality}

Analysis of survey data revealed that participants did not perceive project criticality to be significantly correlated with SDM. This statistical result does not support the research conducted by Boehm and Turner (2003) and others who argue that highly critical projects are better managed with traditional approaches as they bring discipline and rigour to the development process. The survey did not confirm this claim and interviewees only gave a medium importance to this variable. This is in agreement with Strode (2005), who found that this variable has no importance in the success of agile project.

One might argue that the lack of statistical evidence for this variable comes from the lack of highly critical projects in the studied database. However, in general, the majority of projects are on average not very critical, and highly critical projects are fairly rare. Although, a few highly critical and successful agile projects were included in the database, the three most critical projects were managed with a traditional plan-driven methodology.

A different perspective on project criticality can justify the use of agile approaches to address critical issues (Turk, France \& Rumpe, 2002). Agile methodologies may reduce the chances of a defect to occur, by continuous testing and pair programming of the most critical features among other practices. In addition, continuous feedback 
from the customer may help to refine critical features that were little understood earlier. Finally, one can maintain that project criticality mostly impacts the level of risk management rather than the selection of a SDM. All the methodologies can arguably be tailored to adapt to an increased level of risk.

\subsubsection{Project size}

Analysis of survey data revealed that participants did not perceive project size to be significantly correlated with SDM. Project size, a proxy to measure the degree of complexity of a project (Burns \& Dennis, 1985), is a controversial factor in the literature. This study supports the claim that project size is not an important factor in methodology selection despite what certain researchers argue (Boehm \& Turner, 2003; Charvat, 2003). This finding supports recent conclusions of research by Livermore (2008), Misra et al. (2009) and the Forrester Report (West \& Grant, 2010) that found that size is not a key decider in methodology selection.

While Boehm and Turner (2004) report difficulties in using agile approaches for large development teams, others have reported success (Harrison, 2003). For example, in the current study, the biggest successful project was in fact an agile project. It had a cost of USD 790 million and a size of 1,260 man years. The customer was very committed, the requirements were very unstable, the team members were highly empowered, and the approach was supported by both executives and the customer. In other words, the agile philosophy was retained despite the imposing size of the project. Nevertheless, the survey participant who reported this project expressed some difficulties in scaling up agile practices to such a big project.

In very large projects, agile methodologies still need small teams to succeed, but a big project team can be divided into several small project teams manageable with an agile approach. At the upper level, the project management methodology will need to be able to manage and coordinate all the teams. Karlstöm and Runeson (2005) suggest that combining agile methodologies with traditional, stage-gate project management methodologies help coordinate development teams. Creating small subteams is essential to foster collaboration and communication between project team 
members. This is why agile methodologies encourage small teams and small numbers of teams per projects (Coram \& Bohner, 2005).

Thus, although agile methodologies can be applied to any suitable projects without consideration of its size, agile practices need to be tailored and scaled up. Therefore, as suggested during interviews, size informs how the methodology should be tailored to a particular project. One of the risks, though, of applying agile methodologies to a large project though is staff turnover. When the project has a long duration, it is more likely that the project will have a large amount of staff turnover, which means a loss of knowledge. The length of the project was not considered in this study but could be an area of investigation for further study.

On the other hand, it is less difficult to apply traditional methodologies to large projects. Authors, on the contrary, express difficulty in applying these methodologies to small projects as they are too rigid and cumbersome. An actual example of a small successful project in the database managed with a waterfall approach, cost USD 15,000 and had a size of three man months. The team members were very experienced, the requirements very stable and the criticality very low. Therefore, similarly to agile methodologies, it was a question of correctly tailoring the approach to the size of the project to find the right balance of rigidity and flexibility.

In summary, even though plan-driven methodologies scale better to larger projects, agile methodologies can also be scaled up to big projects. Project size is therefore not a contingency factor in SDM fit. It should be noted that although project size does not appear to be an important contingency factor, co-location of the team members is, as it is essential to facilitate communication between team members.

\subsubsection{Experience level of the team}

Analysis of survey data revealed that participants did not perceive experience level of the team to be significantly correlated with SDM. Many researchers consider the experience level of the team (or competence) as a necessary condition in the adoption of an agile methodology. This study did not identify any significant contribution of 
this factor to SDM fit. However, as discussed in a later paragraph, the experience level of the team directly contributes to project success regardless of the SDM used.

This factor is actually complex and in the survey only the average level of experience of the team was measured. It can be hypothesized that the experience of the team leader and/or project manager in particular has a more significant impact on the team, which was not measured in this survey.

Boehm and Turner's (2003) personnel factor is also more complex than the way experience level of the team was measured in the current study. They do not claim that agile projects need experienced people while plan-driven projects only need less experienced people. They assert instead that agile projects need a minimum ratio of experienced personnel over less experienced ones. As far as traditional projects are concerned, they argue that any level of experience can easily be accommodated. Thus the experience level as such is not a key contingency factor in methodology selection. It is rather a prerequisite factor for agile methodologies although this is not confirmed by the present study. In the database, $20 \%$ of the successful agile projects were conducted with a team that had, on average, little experience.

Agile methodologies actually emphasise the role of people (Cockburn \& Highsmith, 2001). Agile methodologies not only need good people, but also people with good interpersonal skills to improve communication among team members. In addition, agile methodologies need generalist team members, while traditional methodologies are based on specialist team members. This distinction was not addressed by the survey and needs further research.

To sum up, the findings of the current study do not support the contention that experience level of the team is an important contingency factor in methodology selection. More important than the skill level, agile teams need agile people; that is, people who can easily adapt themselves, who do not need to be told what to do, and who are good communicators. 


\subsection{SDM and SDM Fit}

The survey showed that most development teams do not use a methodology in its pure form. This result is similar to the Forrester report that shows that $27 \%$ of organizations that have adopted agile methodologies stick to a particular agile methodology while others mix it with other agile or non-agile methodologies (West \& Grant, 2010).

Consequently, SDM fit is not only about selecting one category of methodology but finding the right balance between agile and plan-driven methodologies that best fit the project. In other words, different degrees of agility are needed for different projects. This confirms Boehm's affirmation (2002, p. 69) that “organizations must carefully evolve toward the best balance of agile and plan-driven methodologies that fits their situation".

Besides, the SDM does not need to be fixed throughout the development process; it may change. A few survey participants gave examples of projects where the SDM changed during the project. This can be the result of major changes in the characteristics of the project or its environment. In other cases, the methodology changes because of a lack of delivery with the current approach. According to the interviews and survey findings, the decision to change methodology often comes from executives.

The initial choice of a methodology is also very often made by top management and most project managers that were interviewed for this study complained that they did not have the ability to choose the best methodology. The methodology is instead imposed without logical justification. Therefore, there is a need to rationalize the methodology selection process to be able to justify the choice of a particular approach.

Finally, this study supports the contention that one size does not fit all and that a contingency approach is an appropriate approach to choosing the methodology that best fits. Each project requires a SDM that fits its characteristics and environment. Methodologies work well in specific circumstances, not in any circumstances. There is, therefore, room for both traditional and agile methodologies to coexist. But in 
fact, this study demonstrates that there are not only two types of projects; the traditional or the agile, but instead many methodologies, which vary on a wide spectrum from plan-driven to agile.

\subsection{Project Success}

There is a philosophical difference between agile and traditional SDMs regarding the way they measure success. This study used a global measure of success that may be acceptable for both communities. Based on this measure, the current research studied the impact of project environment, SDM fit, and project on project success. While the PMBOK (PMI, 2008) mentions dozens of factors that influence a project's success, this study identified three that have a particularly important influence and explain nearly a quarter of project success. One of them is SDM fit. No previous study that measured the impact of SDM fit on project success was identified. This important finding implies that SDM should be carefully chosen to fit the project and thereby enhance project success. Nevertheless, SDM fit does not guarantee project success as other unrelated factors may impact on the outcome of the project too. A few unsuccessful projects in the database had a high level of SDM fit. These are projects for which other factors have influenced the outcome of the project.

This study also demonstrates that one size does not fit all, as there is no relationship between SDM and project success. In other words, the use of a particular methodology regardless of the characteristics of the project or its environment does not improve project success. Tiwana and Keil (2004) also found that the use of a potentially inappropriate methodology (i.e., one chosen without consideration of the project context) was a major risk driver. The current study also demonstrated that the one-size-fits-all approach is inappropriate. This mentality can lead to bad methodology choices that threaten the chances of project success.

This study demonstrates that two other factors have an impact on project success: experience level of the team and market uncertainty. A more experienced team will therefore increase the success of the project regardless of the methodology chosen. Similarly, market uncertainty has a direct impact on project success. The more stable 
the market is, the more likely the project will be successful. These two factors do not influence SDM fit, but directly impact project success.

Finally, a separate analysis revealed that technological and project uncertainty also significantly negatively correlate with project success (see section 4.2.8.1). In brief, the more stable the project is in terms of market, technology and requirement, the more likely it is to be successful.

\subsection{Conclusion}

This chapter discussed the findings of the current study and addressed questions concerning the five research gaps (Table 2-5). The current study reveals that both the project and project environment have a critical role in determining the SDM that fits, despite the focus of many researchers on only the project factors. In addition, SDM fit, project environment and project, all play an important role in determining project success. The role of SDM fit in project success has often been neglected in the project success literature and the current study provides evidences that more attention is needed on this aspect. 


\section{CHAPTER 6: CONCLUSION}

This chapter concludes this report with a summary of the key findings and responses to the research questions. It relates the findings to the purpose of the research, acknowledges the limitations of this study, states the implications and gives recommendations for future research.

\subsection{Responses to the Research Questions}

There is an increasing diversity of project types and SDMs. However, frameworks or theories of SDM fit that connect the two are limited. This research addressed this problem by first reviewing the literature on the two categories of SDMs. A definition of SDM fit in the context of this research was provided, as follows: SDM fit is the choice of a methodology that delivers project success in the context of relevant factors associated with the project and project environment. Then, the contingency factors identified in the literature were evaluated by interviews of project workers and experts, and then measured in a survey. The analysis of the data collected during the interviews and survey provided responses to the two research questions posed in chapter 1:

- What are the critical factors in SDM fit?

- What is the role of SDM fit in project success?

The responses to these questions are summarized below.

\subsubsection{Critical factors in SDM fit}

Two critical factors in SDM fit were identified by the current study. A statistical measure of the importance of these two factors was obtained by multiple regression analysis (see Table 4-26). Figure 6-1 shows the standardized regression coefficients between these two factors and a SDM that fits. SDMs are measured on a spectrum from plan-driven to agile. 


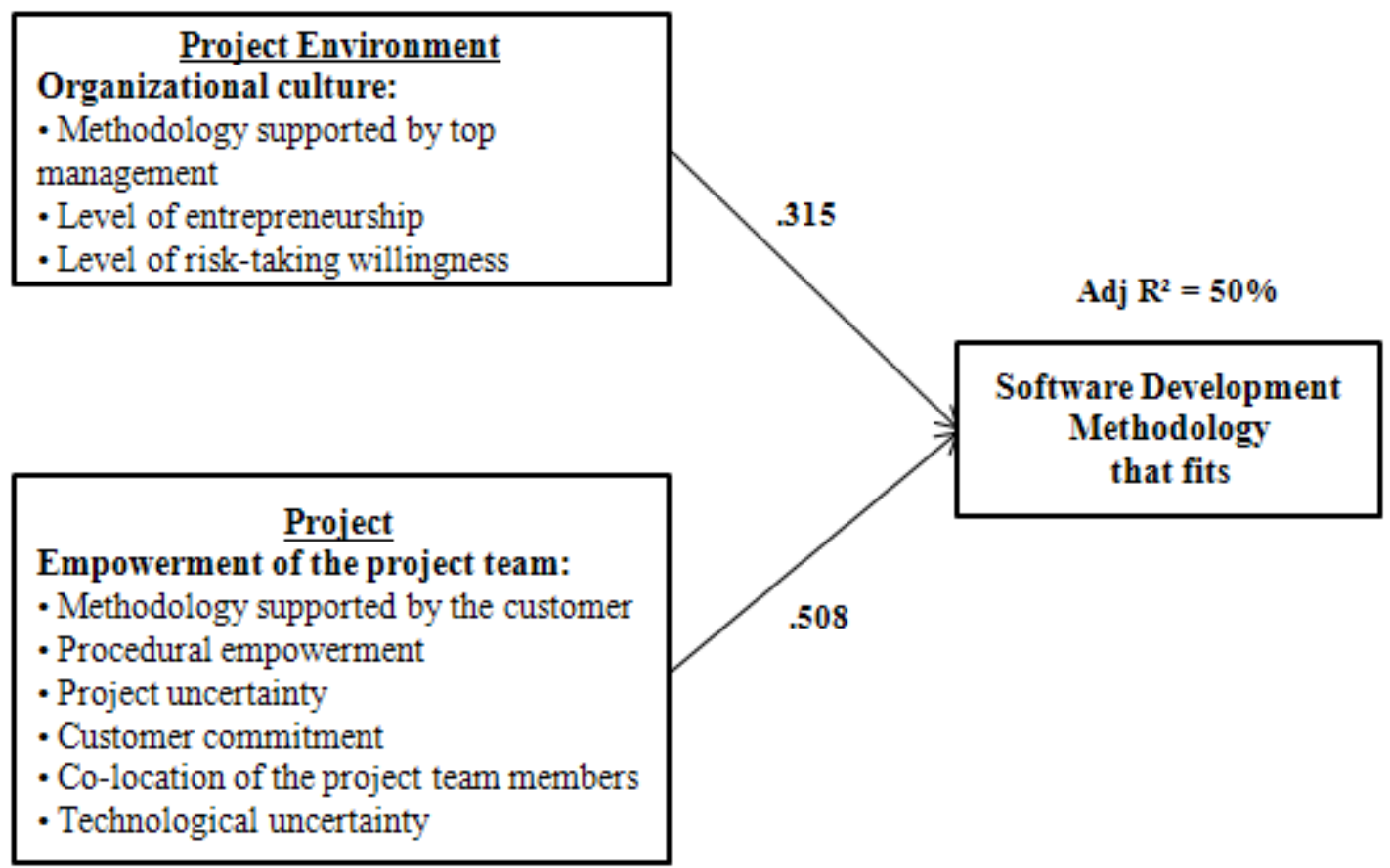

Figure 6-1: Critical factors in SDM fit

The most important factor is related to the characteristics of the project and was named 'Empowerment of the project team'. This factor includes six variables (defined in Appendix F). Listed in descending order of importance, these are: methodology supported by the customer, procedural empowerment, project uncertainty, customer commitment, co-location of the project team members, and technological uncertainty. When the customer supports a plan-driven SDM and the remaining five variables have a low score (i.e. low procedural empowerment, low project uncertainty, low customer commitment, low co-location of the project team members, and low technological uncertainty), then a plan-driven approach best fits the project. Similarly, if the customer supports an agile software development approach, and the remaining five variables have a high score, then an agile approach is most appropriate.

The second most important factor is related to the project environment and was named 'Organizational culture'. It includes methodology supported by top management, level of entrepreneurship, and level of risk-taking willingness (defined in Appendix F). Agile SDMs work best in an entrepreneurial environment, while plan-driven approaches are more likely to succeed in a conservative environment. 
'Empowerment of the project team' and 'Organizational culture' explain 50\% of the variability in the choice of a SDM that fits. No evidence was found that other commonly cited variables in the literature such as complexity, project criticality, and project size have a significant role in SDM fit.

\subsubsection{Role of SDM fit in project success}

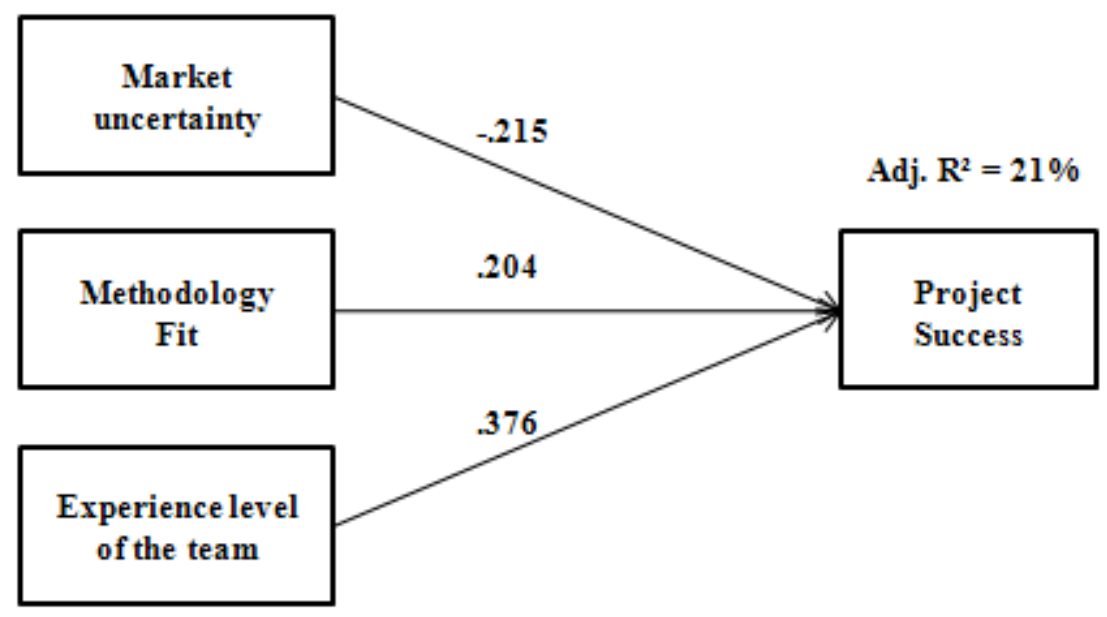

Figure 6-2: Critical factors in project success

The measure of SDM fit is significantly and positively correlated with measures of project success, and makes a statistically significant contribution to project success, thus supporting our hypotheses that SDM fit has impact on project success (Figure 6-2). Nevertheless, the ability to choose the most appropriate SDM is not the only factor that has a positive impact on the outcome of the project. The two other factors highlighted by this research are the experience level of the team and market uncertainty. Experience has a positive impact, while market uncertainty has a negative impact. The regression analysis revealed that together, these three factors explain more than a fifth $(21 \%)$ of the variability in project success.

It can therefore be concluded that a contingency approach based on the factors identified by this research may assist practitioners in selecting the most appropriate methodology, which will in turn result in higher rates of project success. 


\subsection{Implications}

The current study has implications for both project management researchers and practitioners.

The implications for project management researchers are numerous. This study's findings show that project environment factors should not be neglected (Figure 6-1). In particular, the role of organizational culture in SDM fit deserves more attention from researchers. In addition, the current research explains why different methodologies with different degrees of agility are needed, depending on the project characteristics and project environment. Also, as Williams and Cockburn (2003) noted, agile methodologies have evolved to be scalable to a much greater variety of project sizes. These methodologies are often mistakenly seen as being applicable to only small and non-critical projects with highly skilled project team-members. The current findings do not support these common assumptions about SDM selection. In particular, project size, criticality, and the experience level of the team commonly described as critical contingency factors were not found to be significant. Research should therefore refocus on other factors such as organizational and project team culture as well as the role of the customer.

The current study has at least four implications for practitioners. First, to obtain better results, practitioners have to ensure that their allegiance to a particular community of project management methodology practice does not blind them to the need to select the most appropriate SDM. Second, the current study demonstrates that when top management, the project management, project team and customer fail to agree on the SDM, project success will suffer. Customers unwilling to engage in an agile project, for instance, will jeopardize the outcome of the project. Third, the findings demonstrate that project managers and, more generally, top management and organizations as a whole should adopt a more project-specific approach to project management and software development. The study provides a metric for evaluating SDM fit based on the variables identified in Figure 6-1. The higher the project score is on these variables, the more appropriate an agile approach is. Fourth, practitioners will have to think about how to tailor the methodology selected to best fit their needs. This is particularly true when these factors do not lead to a clear 
choice of methodology. The use of a hybrid methodology that combines the features of plan-driven and agile methodologies may be needed in certain cases.

\subsection{Contribution of this Research}

Previous (qualitative, exploratory) research has started to develop agreement on the project factors determining methodology selection. The current research contributes to the SDM fit literature a contingency model that includes the impact of factors associated with the project and the project environment, on SDM fit and project success.

Thus one of the main contributions of this research is the identification of some of the important contingency factors in SDM fit. A second contribution is the identification of the impact of SDM fit on project success. This is a step towards the development of a decision tool that would help project managers or executives choose the most appropriate SDM. The contingencies identified and evaluated by this research may assist practitioners to select the most appropriate methodology, and to achieve higher project success rates.

No support was found for claims such as agile methodologies are inappropriate for large or critical projects. Thus new insights into agile methodologies are provided by this research, which shows that agile methodologies have evolved over time. In particular, this study provides support that agile SDMs are no longer restricted to small and non-critical projects.

Finally, this study found no significant correlation between SDM and project success. This statistically supports the common claim that 'one size does not fit all'.

\subsection{Limitations}

This study has several limitations, which are detailed below.

First, due to the lack of literature in SDM fit and particularly the lack of quantitative instruments in SDM fit, this research is exploratory. Second, a survey was used to 
find statistical evidence for the contingency factors. However, this instrument has its limitations. The measured variables are complex and the questions used to measure them do not fully capture the complexity of the real world. Thus the findings presented in this study only relate to the dimensions measured in the survey. Third, the constructs used are not all based on unequivocal conceptualizations and validated instruments. The large number of constructs and the need for a brief survey, mandated that many constructs were measured by a single question

The way SDM is evaluated on a spectrum from traditional to agile is not based on any existing instrument, which limits the validity of the findings. Even though agile methodologies, for example share the same underlying philosophies, they do not all use the same methods and techniques. New measures had to be devised to measure this construct. Questions on the usage by the project team of techniques and practices within the same category may have provided more accurate measures of SDMs. However, this would have increased the length of the survey.

The use of a survey may also have introduced a self-bias from respondents who would not have responded honestly. It has been suggested that agile people would say that project success is high and that the customer is satisfied because this is what agile methodologies are supposed to bring. What is more, the measure of project success influences, importantly, the contingency factors in SDM fit. The questions used to measure project success address a broad spectrum of project success dimensions, but other ways of measuring project success may have given different findings. This suggests a more detailed analysis of the study data is required to link components of independent project environment and project variables to components of project success.

In this study, the sample is not entirely random, which may limit the generalizability of the findings. Although a large variety of projects was represented in the database, a survey of different projects in different countries and environments may have produced slightly different outcomes. Also, the sample size remains relatively low. A larger sample size could have provided more accurate statistical evidence. Nevertheless, the goal of this exploratory study was not the generalization of the findings. 
Finally, this research assumes that different respondent positions do not influence the data collected. It would have been interesting to see if there are any significant differences according to the respondent's position. Programme managers, for example, may have insights that differ from those of project managers or customers.

\subsection{Future research}

This study is a non-negligible exploratory step in the development of a theory of SDM fit. Additional analysis of the study data will be conducted. More research seems appropriate to confirm the results and to expand the scope of the study.

Confirmation of the results could proceed via qualitative research employing semistructure interviews, for example, to look more carefully into the impact of role (for example, programme manager, project manager, customer) on SDM fit and project success.

Further quantitative research, using a closely similar survey instrument, could be undertaken to validate and confirm the results obtained in this study. Such future research would need to use several questions for each variable for increased accuracy. The refined survey instrument should be applied in different countries (particularly in Asia) to test the power distance variable, and the impact of the national culture on SDM fit and project success. Quantitative analysis could also evaluate the impact of SDM fit on each variable associated with project success.

Expanding the scope of study could be achieved, for example, by the development of a contingency framework of SDM selection that is more closely integrated with research on project success. The factors identified in the current research would constitute a major part of the expanded study. The continued rationalization of methodology selection would provide project managers with a framework and associated metrics to justify to their executives or customers the most appropriate approach. Case study research could then test the applicability and usefulness of the framework in practice. 


\section{REFERENCES}

Agile Alliance. (2001). Manifesto for Agile Software Development. Retrieved November 16, 2009 from www.agilealliance.org

APM. (2006). APM Body of Knowledge (5th ed.). Buckinghamshire: Association for Project Management.

Atkinson, R., Crawford, L, \& Ward, S. (2006). Fundamental uncertainties in projects and the scope of project management. International Journal of Project Management, 24 (8), 687-698.

Avison, D. E., \& Fitzgerald, G. (1995). Information Systems Develoment: Methodologies, Techniques and Tools. Berkshire, England: McGraw-Hill Publishing Company.

Beck, K. (2000). Extreme Programming Explained. Reading, MA: Addison Wesley.

Boehm, B. (2002). Get Ready for Agile Methods, with Care. Computer, 35 (1), 6469.

Boehm, B., \& Turner, R. (2003). Using Risk to Balance Agile and Plan-Driven Methods. IEEE Computer Society, 36 (6), 57-66.

Boehm, B., \& Turner, R. (2004). Balancing Agility and Discipline: A guide for the Perplexed. Addison-Wesley.

Boehm, B., \& Turner, R. (2005). Management Challenges to Implementing Agile Processes in Traditional Development Organizations. IEEE software, 22 (5), 30-39.

Burns, R. N., \& Dennis, A. R. (1985). Selecting the Appropriate Application Development Methodology. ACM SIGMIS Database, 17 (1), 19-23.

Ceschi, M., Sillitti, A., Succi, G., \& De Panfilis, S. (2005). Project Management in Plan-Based and Agile Companies. IEEE software, 22 (3), 21-27.

Charvat, J. (2003). Project Management Methodologies: Selecting, Implementing and Supporting Methodologies and Processes for Projects. New York: John Wiley \& Sons, Inc.

Chow, T., \& Cao, D. (2008). A Survey of Critical Success Factors in Agile Software Projects. The Journal of Systems and Software, 81 (6), 961-971.

Cockburn, A. (2000). Selecting a Project's Methodology. IEEE software, 17 (4), 6471.

Cockburn, A. (2002). Agile Software Development. Upper Saddle, NJ: AddisonWesley. 
Cockburn, A. (2007). Agile Software Development: The Cooperative Game. Upper Saddle River, NJ: Addison-Wesley.

Cockburn, A., \& Highsmith, J. (2001). Agile Software Development: The People Factor. Computer, 34 (11), 131-133.

Collis, J., \& Hussey, R. (2003). Business Research : A Practical Guide for Undergraduate and Postgraduate Students. New York: Palgrave Macmillan.

Cooke-Davies, T. (2002). The "Real" Success Factors on Projects. International Journal of Project Management, 20 (3), 185-190.

Coram, M., \& Bohner, S. (2005). The Impact of Agile Metods on Software Project Management. Proceedings of the 12th IEEE International Conference and Workshops on the Engineering of Computer-Based Systems (pp. 363-370). EEE Computer Society: Washington, DC, USA.

Dalcher, D., \& Brodie, L. (2007). Successful IT Projects. London: Thomson Learning.

DOI. (2005). Declaration of Interdependence. Retrieved January 18, 2010 from http://pmdoi.org/

Dvir, D., \& Shenhar, A. J. (1992). Measuring the Success of Technology Based Strategic Business Units. Engineering Management Journal, 4 (4), 33-38.

Dybå, T., \& Dingsøyr, T. (2008). Empirical Studies of Agile Software Development: A Systematic Review. Information and Software Technology, 50 (9-10), 833859.

Dybå, T., \& Dingsøyr, T. (2009). What do we know about Agile Software Development. IEEE Software, 26 (5), 6-9.

Dybå, T., Moe, N. B., \& Arisholm, E. (2005). Measuring Software Methodology Usage: Challenges of Conceptualization and Operationalization. ISESE, (pp. 447-457). Noosa Heads, Australia.

Easterby-Smith, M., Thorpe, R., \& Jackson, P. R. (2008). Management Research (3rd ed.). Los Angeles: Sage.

Elkington, P., \& Smallman, C. (2002). Managing Project Risks: a Case Study from the Utilities Sector. International Journal of Project Management, 20 (1), 4957.

Eva, M. (1994). SSADM Version 4: A User's Guide (2nd ed.). London: McGraw-Hill Book Company.

Faiks, A., \& Hyland, N. (2000). Gaining User Insight: A Case Study Illustrating the Card Sort Technique. College and research libraries, 61 (4), 349-357.

Freeman, M., \& Beale, P. (1992). Measuring Project Success. Project Management Journal, 23 (1), 8-17. 
Geraldi, J.G., Lee-Kelley, L., and Kutsch, E. (2010). The Titanic sunk, so what? Project manager response to unexpected events. International Journal of Project Management, 28, 547-558.

Graham, I., Henderson-Sellers, B., \& Younessi, H. (1997). The OPEN Process Specification. Harlow, England: Addison-Wesley.

Hardgrave, B. C., Wilson, R. L., \& Eastman, K. (1999). Toward a Contingency Model for Selecting and Information Prototyping Strategy. Journal of Management Information Systems, 16 (2), 113-136.

Harrison, N. B. (2003). A Study of Extreme Programming in a Large Company. Retrieved April 12, 2010, from http://www.agilealliance.org/system/article/file/1292/file.pdf

Highsmith, J., \& Cockburn, A. (2001). Agile Software Develpment: The Business of Innovation. Computer, 34 (9), 120-127.

Highsmith, J. (2002). Agile Software Development Ecosystems. Boston: AddisonWesley.

Highsmith, J. (2010). Agile Project Management: Creating Inovative Products (2nd ed.). Upper Saddle River, NJ: Addison-Wesley.

Hofstede, G. H., \& Hofstede, G. J. (2005). Cultures and Organizations: Software of the Mind (2nd ed.). New York: McGraw-Hill.

Howell, D., Windahl, C., \& Seidel, R. (2010). A Project Contingency Framework based on Uncertainty and its Consequences. International Journal of Project Management, 28 (3), 256-264.

Huisman, M., \& Iivari, J. (2006). Deployment of Systems Development Methodologies: Perceptual Congruence between IS Managers and Systems Developers. Information and Management, 43 (1), 29-49.

Iivari, J., \& Huisman, M. (2007). The Relationship between Organizational Culture and the Deployment of Systems Development Methodologies. MIS Quaterly, 31 (1), 35-58.

Jacobson, I., Booch, G., \& Rumbaugh, J. (1999). The Unified Software Development Process. Reading, Massachusetts: Addison-Wesley.

Jugdev, K., \& Muller, R. (2005). A Retrospective Look at our Evolving Understanding of Project Success. Project management journal, 36 (4), 19-31.

Karlström, D., \& Runeson, P. (2005). Combining Agile Methods with Stage-Gate Project Management. IEEE Software, 22 (3), 43-49.

Koch, A. S. (2005). Agile Software Development: Evaluating the Methods for Your Organization. Boston: Artech House. 
Leffingwell, D. (2007). Scaling Software Agility: Best Practices for Large Enterprises. Upper Saddle River, NJ: Addison Wesley.

Lipovetsky, S., Tishler, A., Dvir, D., \& Shenhar, A. (1997). The Relative Importance of Project Success Dimensions. R\&D Management, 27 (2), 97-106.

Little, T. (2005). Context-Adaptive Agility: Managing Complexity and Uncertainty. IEEE Software, 22 (3), 28-35.

Livermore, J. A. (2008). Factors that Significantly Impact the Implementation of an Agile Software Development Methodology. Journal of Software, 3 (4), 31-36.

Luftman, J., \& Ben-Zvi, T. (2010). Key Issues for IT Executives 2009: Difficult Economy's Impact on IT. MIS Quartely Executive, 9 (1), 49-59.

Martin, J., \& Finkelstein, C. (1981). Information Engineering (Vol. 1 and 2). Englewood Cliffs, New Jersey: Prentice Hall.

McConnell, S. (1996). Rapid Development: Taming Wild Software Schedules. Redmond, Washington: Microsoft Press.

Misra, S. C., Kumar, V., \& Kumar, U. (2009). Identifying some Important Success Factors in Adopting Agile Software Development Practices. Journal of Systems and Software, 82 (11), 1869-1890.

Mohagheghi, P. (2008). Evaluating Software Development Methodologies based on their Practices and Promises. In Proc. Somet'08: New Trends in Software Methodologies, Tools and Techniques (pp. 14-35). IOS Press.

Nerur, S., Mahapatra, R., \& Mangalaraj, G. (2005). Challenges of Migrating to Agile Methodologies. Communications of the ACM, 48 (5), 73-78.

Office of Government Commerce. (2009). Managing Successful Projects with PRINCE2. London: TSO.

O'Leary, Z. (2004). The Essential Guide To Doing Research. Los Angeles: Sage.

Papke-Shields, K.E., Beise, C., \& Quan, J. (2010). Do project managers practice what they preach, and does it matter to project success ? International Journal of Project Management, 28, 650-662.

Pellegrinelli, S. (2010). What's in a name: Project or programme? International Journal of Project Management, Article in Press.

Perrin, R. (2008). Real-world Project Management: Beyond Conventional Wisdom, Best Practices, and Project Methodologies. Wiley.

Pixton, P., Nickolaisen, N., Little, T., \& McDonald, K. (2009). Stand Back and Deliver: Accelerating Business Agility. Upper Saddle River, NJ: AddisonWesley. 
PMI. (2008). A Guide to the Project Management Body of Knowledge (Pmbok Guide) (4th ed.). Project Management Institute.

Ratbe, D., King, W. R., \& Kim, Y.-G. (2000). The Fit between Project Characteristics and Application Development Methodologies: A Contingency Approach. Journal of Computer Information Systems, 40 (2), 26-33.

Riemenschneider, C. K., Hardgrave, B. C., \& Davis, F. D. (2002). Explaining Software Developer Acceptance of Methodologies: A Comparison of Five Theoritical Models. IEEE Transactions on Software Engineering, 28 (12), 1135-1145.

Rugg, G., \& McGeorge, P. (1997). The Sorting Techniques: A Tutorial on Card Sorts, Picture Sorts and Item Sorts. Expert Systems, 14 (2), 349-357.

Sauser, B. J., Reilly, R. R., \& Shenhar, A. J. (2009). Why Projects Fail? How Contingency Theory Can Provide New Insights - A Comparative Analysis of NASA's Mars Climate Orbiter Loss. International Journal of Project Management, 27 (7), 665-679.

Schwaber, K., \& Beedle, M. (2002). Agile Development with Scrum. Upper Saddle River, NJ: Prentice-Hall.

Sheffield, J. (2005). Systemic Knowledge and the V-model. International Journal of Business Information Systems, 1 (1/2), 83-101.

Sheffield, J., \& Lemétayer, J. (2010). Measuring the Critical Success Factors in Project Management Methodology Fit. PMI Global Congress Proceedings. Melbourne, Australia.

Shenhar, A. J. (2001). One Size Does Not Fit All Projects: Exploring Classical Contingency Domains. Management Science, 47 (3), 394-414.

Shenhar, A. J., Tishler, A., Dvir, D., Lipovetsky, S., \& Lechler, T. (2002). Refining the Search for Project Success Factors: A Multivariate, Typological Approach. $R \& D$ Management, 32 (2), 111-126.

Shenhar, A., \& Dvir, D. (2007). Reinventing Project Management: The Diamond Approach to Successful Growth and Innovation. Boston, MA: Harvard Business School Press.

Spencer, D., \& Warfel, T. (2004, April 07). Card Sorting: A Definitive Guide. Retrieved February 15, 2010, from Boxes and Arrows: http://www.boxesandarrows.com/view/card_sorting_a_definitive_guide

Stapleton, J. (1997). DSDM, Dynamic Systems Development Method: The Method in Practice. Harlow, England: Addison Wesley.

Straub, D., Boudreau, M.-C., \& Gefen, D. (2004). Validation Guidelines for IS Positivist Research. Communications of the Association for Information Systems, 13, 380-427. 
Strode, D. E. (2005). The Agile Methods: An Analytical Comparison of Five Agile Methods and an Investigation of their Target Environment. (Master's thesis, Massey University, New Zealand). http://hdl.handle.net/10179/515.

Strode, D. E., Huff, S. H., \& Tretiakov, A. (2009). The Impact of Organizational Culture on Agile Method Use. 42nd Hawaii International Conference on System Sciences (pp. 1-9). Hawaii: HICSS.

Tesch, D., Sobol, M.G., Klein, G., \& Jiang, J.J. (2009). User and developer common knowledge: Effect on the success of information system development projects. International Journal of Project Management, 27, 657-664.

Takeuchi, H., \& Nonaka, I. (1986). The New New Product Development Game. Harvard Business Review, 64, 137-146.

The Standish Group. (2001). The Chaos Report. West Yarmouth, MA.

Tiwana, A., \& Keil, M. (2004). The One-Minute Risk Assessment Tool. Communications of the ACM, 47 (11), 73-77.

Tortamış, P. I. (2004). Light vs. Heavy: Which To Choose? IMPROQ 2004: Impact of Software Process on Quality Workshop (pp. 6-9). Turkey: Ege Universitesi.

Turk, D., France, R., \& Rumpe, B. (2002). Limitations of Agile Software Processes. Proceedings of the Thrid International Conference on eXtreme Programming and Agile Processes in Software Engineering, (pp. 43-46). Alghere, Sardinia, Italy.

Turner, R., \& Boehm, B. (2003). People Factors in Software Management: Lessons From Comparing Agile and Plan-Driven Methods. The Journal of Defense Software Engineering (Dec), 4-8.

Van Donk, D. P., \& Molloy, E. (2008). From Organising as Projects to Projects as Organizations. International Journal of Project Management, 26 (2), 129-137.

Vinekar, V., Slinkman, C. W., \& Nerur, S. (2006). Can Agile and Traditional Systems Development Approaches Coexist? An Ambidextrous View. Information systems management, 23 (3), 31-42.

Wagner, W. E. (2010). Using SPSS for Social Statistics and Research Methods (2nd ed.). Los Angeles: SAGE Publications.

West, D., \& Grant, T. (2010). Agile Development: Mainstream Adoption Has Changed Agility. Cambridge, MA: Forrester Research.

Williams, L., \& Cockburn, A. (2003). Agile Software Development: It's about Feedback and Change. IEEE Computer Society, 39-43.

Winter, M., Smith, C., Morris, P., \& Cicmil, S. (2006). Directions for Future Research in Project Management: the Findings of a UK Government-Funded Research Network. International Journal of Project Management, 24 (8), 638649. 
Wysocki, R. K. (2006). Effective Software Project Management. Indianapolis, IN: Wiley.

Wysocki, R. R. (2009). Effective Project Management: Traditional, Agile, Extreme (5th ed.). Indianapolis, IN: Wiley. 


\section{APPENDICES}

Appendix A: Information sheet given to interview participants 130

Appendix B: Research agreement signed by interviewees

Appendix C: Interview recording sheet 132

Appendix D: Information sheet sent to survey sample 133

Appendix E: Survey questions 134

Appendix F: Variables 137 


\section{Appendix A: Information sheet given to interview participants}

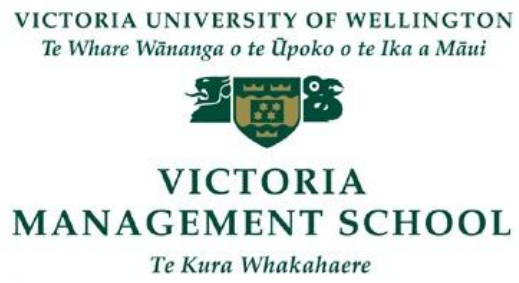

\section{Research project: Measuring the Critical Success Factors in Development Methodology Fit}

I am a master's student at Victoria University of Wellington in New Zealand and am conducting research on IT project management. This research is being conducted as part of the requirements for the completion of my degree (Master of Management Studies).

Many IT projects still fail to deliver on time or on budget, or fail to deliver value to the client. One reason for this is the choice of an inappropriate project management approach. On the basis that one size does not fit all, I'm investigating how to match the methodology chosen to the project characteristics. In particular, the aim of this interview is to determine the most important factors in methodology selection.

\section{Terms and conditions}

- Participation is entirely voluntary.

- If you agree, the interview will be about 30 minutes long and scheduled at a time that suits you.

- You have the right to withdraw yourself or any information you have provided from this project, without having to supply a reason for doing so, for four weeks after the interview. In which case, information obtained will be immediately destroyed.

- Participants will be interviewed confidentially. All information gathered in these interviews will be treated confidentially - your name will not be used. The results from the interviews will be reported in an aggregated, non-attributable form.

- Ethical approval from Victoria University of Wellington has been obtained for the proposed research.

- All participants will sign a Research Agreement where they can state how they would like the data collected from them to be handled.

- A copy of the research paper or thesis will be deposited in the Victoria University of Wellington Library. Findings may be presented at conferences or published in academic or professional journals at a later date. Any further use will require your written consent.

- A summary of the results will be available to participants who ask for it.

\section{Contact Information}

Thank you for your time and help to make this study possible. If you have any queries please do not hesitate to contact me or my supervisor, Dr Jim Sheffield.

Researcher: Julien Lemétayer

Victoria Management School

Victoria University of Wellington
Supervisor: Dr Jim Sheffield

Victoria Management School

Victoria University of Wellington 


\section{Appendix B: Research agreement signed by interviewees}

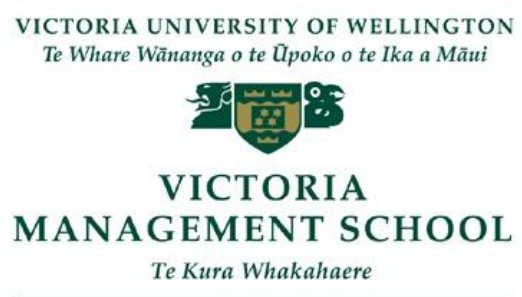

\section{Research Title:}

Measuring the Critical Success Factors in Development Methodology Fit

\section{Researcher:}

Julien Lemétayer, Victoria Management School, Victoria University of Wellington

\section{Purpose of agreement:}

This agreement is to ensure that you are sufficiently informed about the purpose of the research, and your right to know how data will be collected, analysed and written up.

\section{Consent to participation}

I have been given and have understood an explanation of this research project.

$\square$ I have had an opportunity to ask any questions and had them answered to my satisfaction.

$\square$ I understand the data collected will remain confidential and will be reported in an aggregated, non-attributable form.

$\square$ I understand that I may withdraw myself or any information I have provided from this project (for four weeks after the interview), without having to supply a reason for doing so. In which case, information obtained will be immediately destroyed.

$\square$ I understand that the information obtained will be stored in a locked cabinet or password-protected file. All interview notes and research materials will be destroyed two years after the research is completed.

$\square$ A copy of the research paper or thesis will be deposited in the Victoria University of Wellington Library. Findings may be presented at conferences or published in academic or professional journals at a later date. Any further use will require my written consent.

$\square$ I agree to participate in this study.

$\square$ I want to receive a summary of the findings of this study.

$\square$ I agree to receive an invitation by email to participate in a survey that will be conducted on the same topic.

\section{Participant}

Name:

Email:

Date:

Signature:

\section{Researcher}

Julien Lemétayer

Victoria University of Wellington

Signature: 


\section{Appendix C: Interview recording sheet}

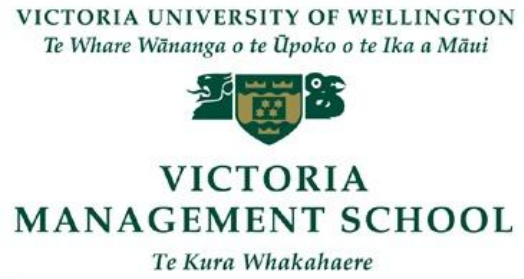

Interview number:

\section{Project factors}

\begin{tabular}{ll}
\hline $\begin{array}{c}\text { Less important factors } \\
\text { (0 points) }\end{array}$ & $\begin{array}{c}\text { More important factors } \\
\text { (1 to } \mathbf{5} \text { points) }\end{array}$ \\
\hline & 5 points (most important): \\
& 4 points: \\
& 3 points: \\
& 2 points: \\
& 1 point (less important): \\
\hline
\end{tabular}

\section{Project environment factors}

\begin{tabular}{ll}
\hline $\begin{array}{c}\text { Less important factors } \\
\text { (0 points) }\end{array}$ & $\begin{array}{c}\text { More important factors } \\
\text { (1 to } 5 \text { points) }\end{array}$ \\
\hline & 5 points (most important): \\
& 4 points: \\
& 3 points: \\
& 2 points: \\
& 1 point (less important): \\
\hline
\end{tabular}




\title{
Appendix D: Information sheet sent to survey sample
}

\author{
VICTORIA UNIVERSITY OF WELLINGTON \\ Te Whare Wānanga o te Ûpoko o te Ika a Mãui

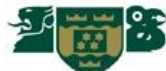 \\ VICTORIA \\ MANAGEMENT SCHOOL \\ Te Kura Whakahaere

\section{International Survey on Software Development Methodology Fit}

You are invited to participate in a survey conducted by researchers at Victoria University of Wellington, New Zealand.

Many IT projects still fail to deliver on time or on budget, or fail to deliver value to the client. One reason for this is the choice of an inappropriate development approach. We are investigating if project success is enhanced by matching the methodology used to the characteristics of the organization and/or project.

The completion of this web-based survey will take less than ten minutes. It is available by clicking on the following link:

\section{http://vuw.qualtrics.com/SE?SID=SV 8jrSkmvwRfD8boE\&SVID=}

I would be most grateful if you could also forward this survey to your friends and colleagues working in software development. Findings are available to all respondents.

If you would like further information about any aspect of this survey, please contact me or my supervisor.

Researcher: Julien Lemétayer

Supervisor: Jim Sheffield, PhD
Julien.Lemetayer@vuw.ac.nz

Jim.Sheffield@vuw.ac.nz

Thank you for your participation and your time,

Julien Lemétayer 


\section{Appendix E: Survey questions}

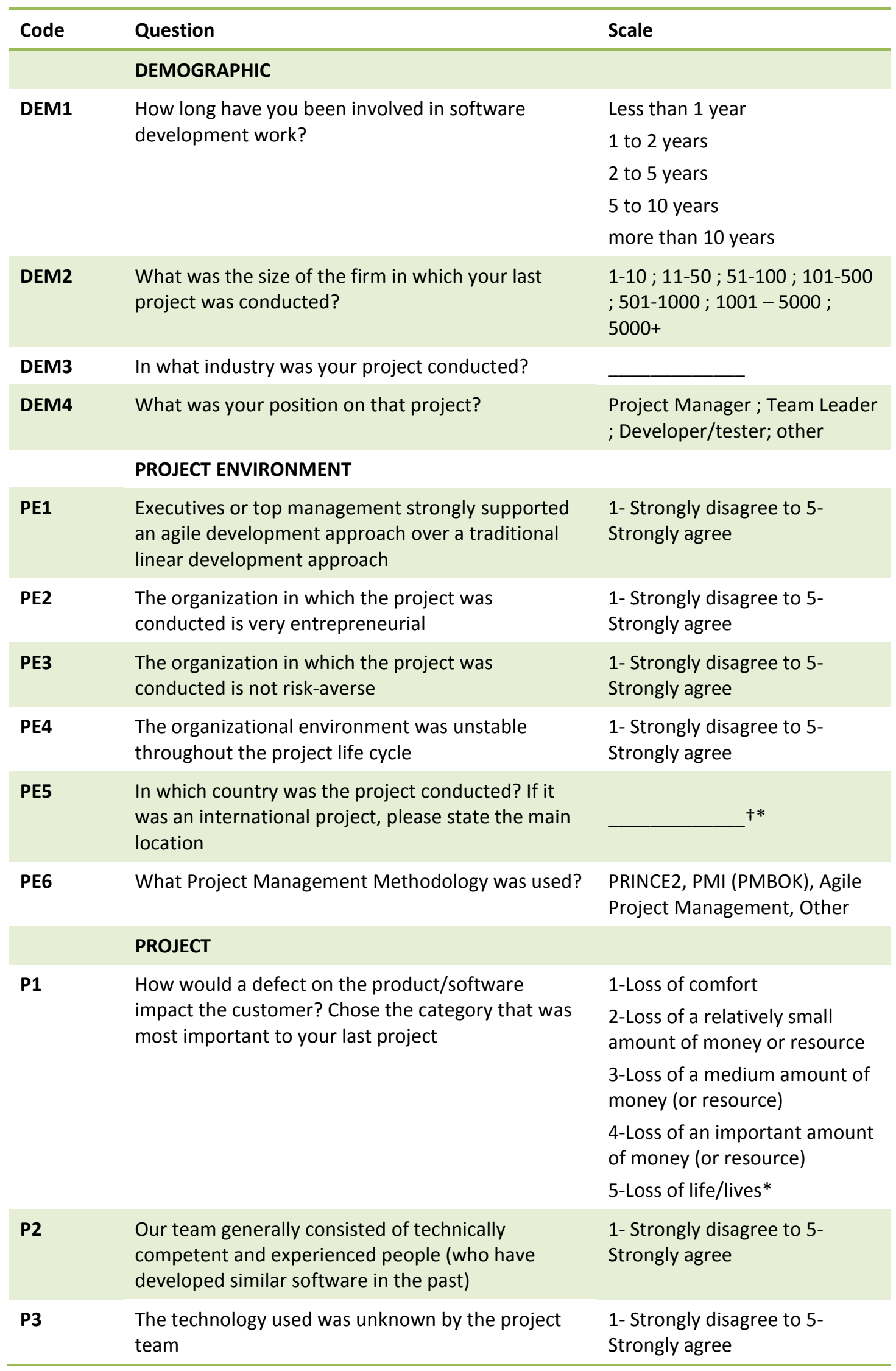




\begin{tabular}{|c|c|c|}
\hline Code & Question & Scale \\
\hline P4 & $\begin{array}{l}\text { The requirements provided to the project team } \\
\text { were unstable throughout the project life cycle }\end{array}$ & $\begin{array}{l}\text { 1- Strongly disagree to } 5 \text { - } \\
\text { Strongly agree }\end{array}$ \\
\hline P5 & $\begin{array}{l}\text { The project team members felt most comfortable } \\
\text { being free to make decisions and empowered }\end{array}$ & $\begin{array}{l}\text { 1- Strongly disagree to } 5 \text { - } \\
\text { Strongly agree }\end{array}$ \\
\hline P6 & $\begin{array}{l}\text { The customer closely collaborated with the } \\
\text { development team members }\end{array}$ & $\begin{array}{l}\text { 1- Strongly disagree to } 5 \text { - } \\
\text { Strongly agree }\end{array}$ \\
\hline P7 & $\begin{array}{l}\text { The customer strongly supported an agile } \\
\text { development approach over a traditional linear } \\
\text { development approach }\end{array}$ & $\begin{array}{l}\text { 1- Strongly disagree to } 5 \text { - } \\
\text { Strongly agree }\end{array}$ \\
\hline \multirow[t]{2}{*}{ P8.1 } & \multirow{2}{*}{$\begin{array}{l}\text { What was the approximate total cost of the } \\
\text { project? (Please choose the appropriate currency) }\end{array}$} & \\
\hline & & $\begin{array}{l}\text { AUD, CAD, EUR, GBP, EUR, } \\
\text { USD, other (please specify) }+\end{array}$ \\
\hline \multirow[t]{2}{*}{ P8.2 } & How many years of work went into that project? & man years \\
\hline & $\begin{array}{l}\text { (e.g. } 3 \text { developers full time for } 2 \text { years = } 6 \text { man } \\
\text { years) }\end{array}$ & Or $\_$man months ${ }^{\dagger}$ \\
\hline \multirow[t]{2}{*}{ P9 } & All the project team members were co-located & $\begin{array}{l}\text { 1- Strongly disagree to } 5 \text { - } \\
\text { Strongly agree }\end{array}$ \\
\hline & \multicolumn{2}{|c|}{ SOFTWARE DEVELOPMENT METHODOLOGY (from plan-driven to agile) } \\
\hline SDM1.1 & $\begin{array}{l}\text { Individuals and interactions were valued more than } \\
\text { processes and tools }\end{array}$ & $\begin{array}{l}\text { 1- Strongly disagree to } 5 \text { - } \\
\text { Strongly agree }\end{array}$ \\
\hline SDM1.2 & $\begin{array}{l}\text { Working software was valued more than } \\
\text { comprehensive documentation }\end{array}$ & $\begin{array}{l}\text { 1- Strongly disagree to } 5 \text { - } \\
\text { Strongly agree }\end{array}$ \\
\hline SDM1.3 & $\begin{array}{l}\text { Close customer collaboration was valued more than } \\
\text { strict adherence to a predetermined contract }\end{array}$ & $\begin{array}{l}\text { 1- Strongly disagree to } 5 \text { - } \\
\text { Strongly agree }\end{array}$ \\
\hline SDM1.4 & $\begin{array}{l}\text { Initiating and responding to change was valued } \\
\text { more than strict adherence to a predetermined plan }\end{array}$ & $\begin{array}{l}\text { 1- Strongly disagree to } 5 \text { - } \\
\text { Strongly agree }\end{array}$ \\
\hline \multirow[t]{5}{*}{ SDM2 } & \multirow{4}{*}{$\begin{array}{l}\text { On which development life cycle was your last } \\
\text { project based on? }\end{array}$} & 1-Linear / Waterfall \\
\hline & & 3-Iterative and incremental \\
\hline & & 5-Agile - iterative and adaptive \\
\hline & & 6-Other (please specify) \\
\hline & \multicolumn{2}{|l|}{ PROJECT SUCCESS } \\
\hline PS1.1 & The project was completed on time or earlier & $\begin{array}{l}\text { 1- Strongly disagree to } 5 \text { - } \\
\text { Strongly agree }\end{array}$ \\
\hline PS1.2 & The project was completed within or below budget & $\begin{array}{l}\text { 1- Strongly disagree to } 5 \text { - } \\
\text { Strongly agree }\end{array}$ \\
\hline PS1.3 & The project met the customer's requirement & $\begin{array}{l}\text { 1- Strongly disagree to } 5 \text { - } \\
\text { Strongly agree }\end{array}$ \\
\hline PS1.4 & The project delivered a good working product & $\begin{array}{l}\text { 1- Strongly disagree to } 5 \text { - } \\
\text { Strongly agree }\end{array}$ \\
\hline PS2.1 & The product addresses a recognized need & $\begin{array}{l}\text { 1- Strongly disagree to } 5 \text { - } \\
\text { Strongly agree }\end{array}$ \\
\hline PS2.2 & The product is used by the customer & $\begin{array}{l}\text { 1- Strongly disagree to 5- } \\
\text { Strongly agree }\end{array}$ \\
\hline
\end{tabular}




\begin{tabular}{lll}
\hline Code & Question & Scale \\
\hline PS2.3 & The product satisfies the customer & $\begin{array}{l}\text { 1- Strongly disagree to 5- } \\
\text { Strongly agree }\end{array}$ \\
PS3.1 & The project team was highly satisfied & $\begin{array}{l}\text { 1- Strongly disagree to 5- } \\
\text { Strongly agree }\end{array}$ \\
PS3.2 & The project team would work the same way again & $\begin{array}{l}\text { 1- Strongly disagree to 5- } \\
\text { Strongly agree }\end{array}$ \\
\hline COM & Any feedback or comments on this study & \\
\hline
\end{tabular}

* Scale was reversed for analysis $\uparrow$ Scale was recoded from 1 to 5 for analysis

Note: In these questions, the customer is the person or organization for whom the software was developed.

Respondents had the possibility to answer 'Don't know' or 'N/A' to all the questions measured on a 5-point Likert scale. 


\section{Appendix F: Variables}

Appendix $\mathrm{F}$ presents information in order by variable cluster (demographic, project environment, project, SDM, project success). Each item consists of a variable code, variable name, and polarity. Descriptive statistics reported in section 4.2.3 apply to each variable as defined here.

For project environment and project variables, the polarity of the response scale has additional significance. For these response scales, the expected directional link ('polarity') between the independent variable and SDM is based on the literature summarised in Table 2-4. Polarities that are asterisked must be reversed after the variable is formed but before the variable is analysed in Sections 4.2.4 through 4.2.8.

\begin{tabular}{|c|c|c|c|}
\hline Code & Variable & Definition & Polarity \\
\hline \multicolumn{4}{|c|}{ PROJECT ENVIRONMENT VARIABLES } \\
\hline PE1 & $\begin{array}{l}\text { Methodology } \\
\text { supported by top } \\
\text { management }\end{array}$ & $\begin{array}{l}\text { Software development methodology } \\
\text { supported by top management (plan-driven } \\
\text { vs. agile) }\end{array}$ & From Plan to Agile \\
\hline PE2 & $\begin{array}{l}\text { Level of } \\
\text { entrepreneurship }\end{array}$ & $\begin{array}{l}\text { Conservative vs. entrepreneurial } \\
\text { organization }\end{array}$ & From Plan to Agile \\
\hline PE3 & $\begin{array}{l}\text { Level of risk-taking } \\
\text { willingness }\end{array}$ & $\begin{array}{l}\text { Level of risk the organization is willing to } \\
\text { take }\end{array}$ & From Plan to Agile \\
\hline PE4 & Market uncertainty & $\begin{array}{l}\text { The environment within which the } \\
\text { organization operates is stable vs. unstable }\end{array}$ & From Plan to Agile \\
\hline PE5 & Power distance & $\begin{array}{l}\text { Acceptance of small vs. large power } \\
\text { differentials (flat vs. tall hierarchy). } \\
\text { Measured by Hofstede's Power Distance } \\
\text { Index }\end{array}$ & From Agile to Plan* \\
\hline PE6 & $\begin{array}{l}\text { Project } \\
\text { Management } \\
\text { Methodology }\end{array}$ & $\begin{array}{l}\text { Traditional linear project management } \\
\text { methodology vs. agile iterative project } \\
\text { management methodology }\end{array}$ & From Plan to Agile \\
\hline PE7 & Economic sector & $\begin{array}{l}\text { Client organization is part of government vs. } \\
\text { private sector }\end{array}$ & From Plan to Agile \\
\hline \multicolumn{4}{|c|}{ PROJECT VARIABLES } \\
\hline P1 & Project criticality & $\begin{array}{l}\text { The level to which a defect in the } \\
\text { product/software would impact the client } \\
\text { (High criticality -> Plan-driven approaches) }\end{array}$ & From Agile to Plan* \\
\hline $\mathrm{P} 2$ & $\begin{array}{l}\text { Experience level of } \\
\text { the team }\end{array}$ & $\begin{array}{l}\text { Experience of the project team members } \\
\text { (this reduces their need for supervision and } \\
\text { guidance) }\end{array}$ & From Plan to Agile \\
\hline P3 & Technological & The technology used is well known by the & From Plan to Agile \\
\hline
\end{tabular}




\begin{tabular}{|c|c|c|c|}
\hline Code & Variable & Definition & Polarity \\
\hline & uncertainty & project team vs. it is totally new & \\
\hline P4 & Project uncertainty & $\begin{array}{l}\text { The user requirements are stable vs. } \\
\text { unstable }\end{array}$ & From Plan to Agile \\
\hline P5 & Empowerment & $\begin{array}{l}\text { The team members feel most comfortable } \\
\text { having clear roles and following clear } \\
\text { policies and procedures vs. they feel most } \\
\text { comfortable being free to make decisions } \\
\text { and empowered. }\end{array}$ & From Plan to Agile \\
\hline P6 & $\begin{array}{l}\text { Customer } \\
\text { commitment }\end{array}$ & $\begin{array}{l}\text { The level of engagement / collaboration the } \\
\text { customer is willing to put in the project }\end{array}$ & From Plan to Agile \\
\hline P7 & $\begin{array}{l}\text { Methodology } \\
\text { supported by the } \\
\text { customer }\end{array}$ & $\begin{array}{l}\text { Software development methodology } \\
\text { supported by the customer (plan-driven vs. } \\
\text { agile) }\end{array}$ & From Plan to Agile \\
\hline P8 & Project size & $\begin{array}{l}\text { Small vs. large project - based on the cost } \\
\text { and size (in man hours) of the project }\end{array}$ & From Agile to Plan* \\
\hline P8.1 & Project Cost & Total cost of the project & From Agile to Plan \\
\hline P8.2 & $\begin{array}{l}\text { Project Size (man } \\
\text { hours) }\end{array}$ & $\begin{array}{l}\text { Estimation of the total amount of } \\
\text { uninterrupted labour required to complete } \\
\text { the project (man-years) }\end{array}$ & From Agile to Plan \\
\hline P9 & $\begin{array}{l}\text { Co-location of the } \\
\text { project team } \\
\text { members }\end{array}$ & $\begin{array}{l}\text { Project team members work in different } \\
\text { sites vs. Project team members work at the } \\
\text { same site }\end{array}$ & From Plan to Agile \\
\hline
\end{tabular}

\section{SOFTWARE DEVELOPMENT METHODOLOGY}

\begin{tabular}{|c|c|c|c|}
\hline SDM1.1 & $\begin{array}{l}\text { Individuals over } \\
\text { Processes }\end{array}$ & $\begin{array}{l}\text { Individuals and interactions were valued } \\
\text { more than processes and tools }\end{array}$ & From Plan to Agile \\
\hline SDM1.2 & $\begin{array}{l}\text { Working code over } \\
\text { documentation }\end{array}$ & $\begin{array}{l}\text { Working software was valued more than } \\
\text { comprehensive documentation }\end{array}$ & From Plan to Agile \\
\hline SDM1.3 & $\begin{array}{l}\text { Collaboration over } \\
\text { contract }\end{array}$ & $\begin{array}{l}\text { Close customer collaboration was valued } \\
\text { more than strict adherence to a } \\
\text { predetermined contract }\end{array}$ & From Plan to Agile \\
\hline SDM1.4 & Change over plan & $\begin{array}{l}\text { Initiating and responding to change was } \\
\text { valued more than strict adherence to a } \\
\text { predetermined plan }\end{array}$ & From Plan to Agile \\
\hline SDM2 & $\begin{array}{l}\text { Development life } \\
\text { cycle }\end{array}$ & $\begin{array}{l}\text { Software development life cycle (linear, } \\
\text { incremental or iterative/adaptive) }\end{array}$ & From Plan to Agile \\
\hline \multicolumn{4}{|c|}{ SOFTWARE DEVELOPMENT METHODOLOGY FIT } \\
\hline FIT & Methodology fit & $\begin{array}{l}\text { Methodology fit is the choice of a } \\
\text { methodology that delivers project success } \\
\text { in the context of relevant factors associated } \\
\text { with the project and project environment }\end{array}$ & $\begin{array}{l}\text { From low } \\
\text { methodology fit to } \\
\text { high methodology } \\
\text { fit }\end{array}$ \\
\hline \multicolumn{4}{|c|}{ PROJECT SUCCESS } \\
\hline PS1.1 & Time & $\begin{array}{l}\text { The project was completed on time or } \\
\text { earlier }\end{array}$ & $\begin{array}{l}\text { From unsuccessful } \\
\text { to successful }\end{array}$ \\
\hline
\end{tabular}




\begin{tabular}{|c|c|c|c|}
\hline Code & Variable & Definition & Polarity \\
\hline PS1.2 & Budget & $\begin{array}{l}\text { The project was completed within or below } \\
\text { budget }\end{array}$ & $\begin{array}{l}\text { From unsuccessful } \\
\text { to successful }\end{array}$ \\
\hline PS1.3 & Functionality & $\begin{array}{l}\text { The project met the customer's } \\
\text { requirement }\end{array}$ & $\begin{array}{l}\text { From unsuccessful } \\
\text { to successful }\end{array}$ \\
\hline PS1.4 & Quality & $\begin{array}{l}\text { The project delivered a good working } \\
\text { product }\end{array}$ & $\begin{array}{l}\text { From unsuccessful } \\
\text { to successful }\end{array}$ \\
\hline PS2.1 & Addresses a need & The product addresses a recognized need & $\begin{array}{l}\text { From unsuccessful } \\
\text { to successful }\end{array}$ \\
\hline PS2.2 & Product is used & The product is used by the customer & $\begin{array}{l}\text { From unsuccessful } \\
\text { to successful }\end{array}$ \\
\hline PS2.3 & $\begin{array}{l}\text { Customer is } \\
\text { satisfied }\end{array}$ & The product satisfied the customer & $\begin{array}{l}\text { From unsuccessful } \\
\text { to successful }\end{array}$ \\
\hline PS3.1 & Team is satisfied & The project team was highly satisfied & $\begin{array}{l}\text { From unsuccessful } \\
\text { to successful }\end{array}$ \\
\hline PS3.2 & $\begin{array}{l}\text { Team would work } \\
\text { the same way } \\
\text { again }\end{array}$ & The team would work the same way again & $\begin{array}{l}\text { From unsuccessful } \\
\text { to successful }\end{array}$ \\
\hline
\end{tabular}

* Scale was reversed before analysis 